\title{
INCARCERATED WOMEN: IMPACT OF CHILDHOOD SEXUAL ABUSE AND \\ FAMILY FUNCTIONING ON \\ DISSOCIATION AND \\ PSYCHOLOGICAL DISTRESS
}

\author{
By \\ MARGARET LEE ZINGMAN, MS
}

Bachelor of Arts

Eckerd College

St. Petersburg, Florida

1979

Master of Science

Oklahoma State University

Stillwater, Oklahoma

1996

Submitted to the Faculty Of The Graduate College Of

Oklahoma State University In Partial Fulfillment

Of The Requirements For The Degree Of

DOCTOR OF PHILOSOPHY

December, 2003 


\section{INCARCERATED WOMEN: IMPACT OF CHILDHOOD SEXUAL ABUSE AND \\ FAMILY FUNCTIONING ON \\ DISSOCIATION AND \\ PSYCHOLOGICAL \\ DISTRESS}

\section{Dissertation approved}
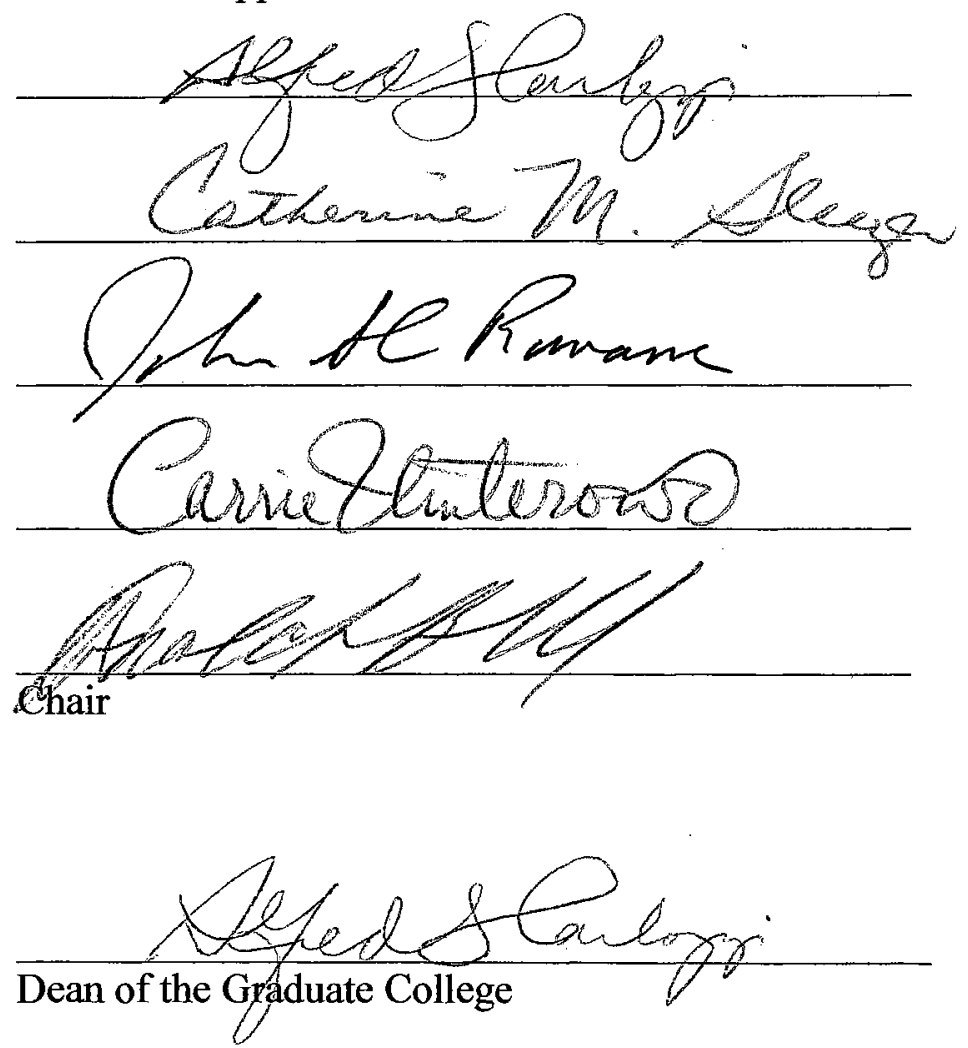


\section{ACKNOWLEDGEMENTS}

I want to acknowledge and thank those who gave me support during the long journey towards my Doctoral degree. My parents, children, friends, brother, and sister, bore the brunt of the weight of these years, yet would never let me give up. I can never repay my mother's emotional support and editing skills, my father's emotional and financial support, my daughter's unfailing ability to make me smile, her sensitivity, her willingness to live as a pauper, my son's sensitivity, his creative air filling the house, his willingness to live as a pauper and my siblings emotional support. I cannot forget Carol Johnson. Without her ongoing assistance, I would have not completed this endeavor. Her unfailing friendship and her astute editing talents brought everything together, including me. In addition, members of my dissertation committee of Donald Boswell, Ph.D., Alfred Carlozzi, Ed. D., Carrie Winterowd, Ph. D., John Romans, Ph. D., and Catharine Sleezer, $\mathrm{Ph}$. D. were more than academic advisors. Each individual knows the part they played, whether it was as a mentor, a provider of emotional support, a technical director, or as a giver of unfailing encouragement. Moreover, without Kirk Bjornsgaard's early editing assistance, this work would never have had a foundation. Thank you! Everyone's unfailing belief in my ability to succeed is why I passed through obstacles that could have prematurely ended this journey. I am forever indebted. 


\section{TABLE OF CONTENTS}

Chapter Page

I. INTRODUCTION

Trauma and Its impact on Mental Health $\ldots \ldots \ldots \ldots \ldots \ldots \ldots$

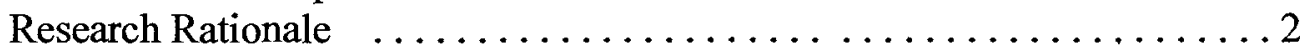

Childhood Sexual Abuse and Symptom Predictors . ............ 4

Developmental Theories and Family Dynamics . . . . . . . . . . . 5

Childhood Family Conflict, Expressiveness and Cohesiveness . . . . . . 7

Dissociation . . . . . . . . . . . . . . . . . 8

Psychological Distress and Dissociation: Reciprocal Relationships . . . . 11

Incarcerated Women . . . . . . . . . . . . . . . . . . . 13

Research Problem ............................ 15

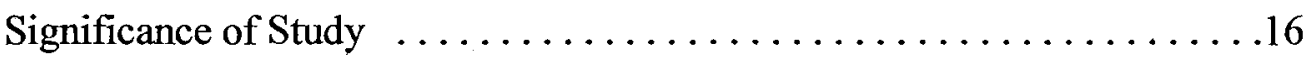

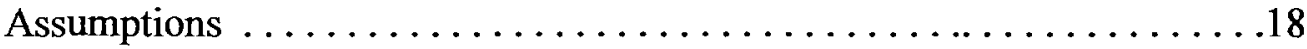

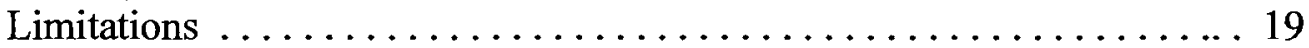

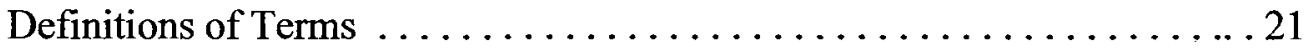

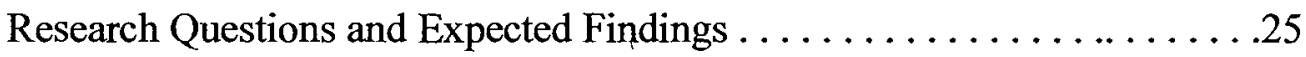

II. LITERATURE REVIEW

Demographic Analysis of Childhood Sexual Abuse Survivors . . . . . . 27

Dissociation . . . . . . . . . . . . . . . . . . . . . 29

Family Functioning, Dissociation, and Psychological Distress . . . . . . 30

Under-researched, Under-treated Populations . . . . . . . . . . . 31

The Impact of Sexual Trauma . . . . . . . . . . . . . . . . . . 32

The Impact of Dissociation. . . . . . . . . . . . . . 35

The Impact of Family Relationship Dynamics . . . . . . . . . . 41

The Impact of Psychological Distress . . . . . . . . . . . . 49

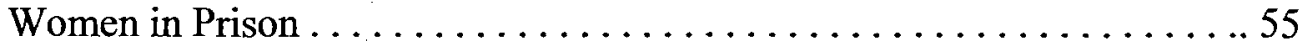

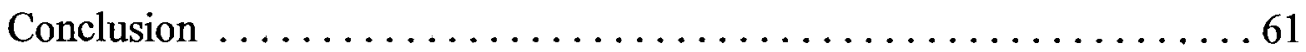

III. METHOD

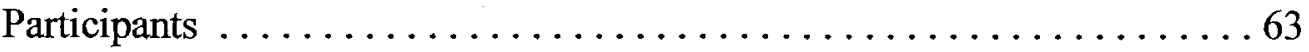

Demographic Characteristics of the Sample .............66 63

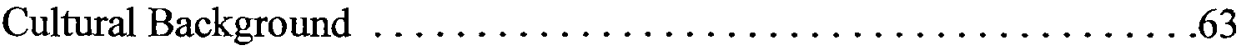

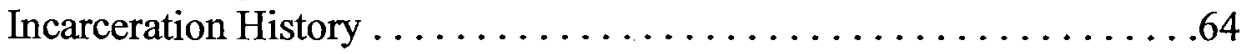

Identified Childhood Caregiver. . . . . . . . . . . . . . 64 
Instrumentation ............................... 64

Background Information Sheet ................... 64

Moos and Moos Family Environment Scale . . . . . . . . . . . . . .65

Dissociative Experiences Scale ........................67

Symptom Checklist -90 -Revised ...................668

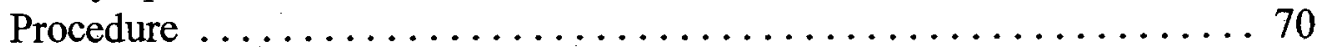

Design of Study . . . . . . . . . . . . . . . . . . . . 72

Independent and Dependent Variables .................... 73

IV. RESULTS

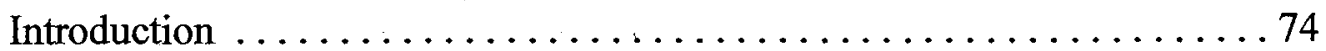

General Analysis Of Results . . . . . . . . . . . . . . . . . . . . 74

Occurrence of Sexual Abuse. ............................ 74

Counseling After the Abuse. ....................... 74

Disclosure Experiences .............................. 75

Breakdown of DES and GSI Score $\ldots \ldots \ldots \ldots \ldots \ldots \ldots \ldots \ldots$

Statistical Analysis .............................. 75

Research Question 1 Analysis (Tables 1-3)

Sexual Abuse by Level of Childhood Family Conflict......... 75

Research Question 2 Analysis (Tables 4-6)

Sexual Abuse by Level of Family Expressiveness . . . . . . . . . 76

Research Question 3 Analysis (Tables 7 - 9)

Sexual Abuse by Level of Family Conflict $\ldots \ldots \ldots \ldots \ldots \ldots 83$

\section{DISCUSSION}

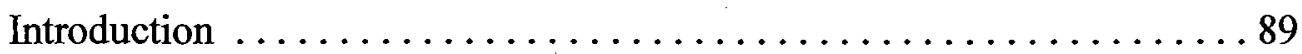

Limitation of the Study . . . . . . . . . . . . . . . . . . . . . 89

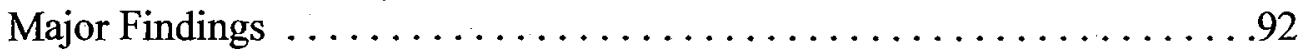

Implications . ................................ 93

Suggestions for Future Research . . . . . . . . . . . . . . . . 108

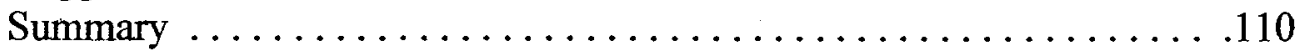

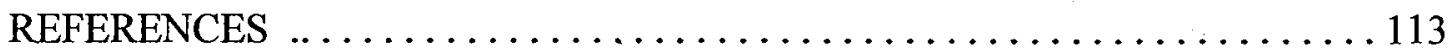

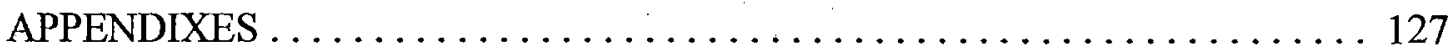

A. Demographic Sheet ............................ 127

B. Informed Consent . . . . . . . . . . . . . . . . . . . . . . . . 131

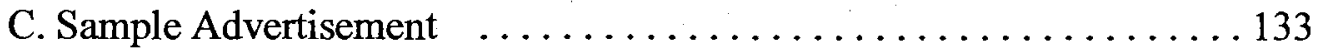

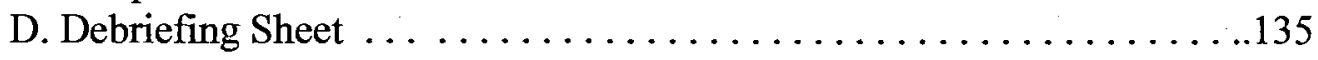

E. Request for Assistance . . . . . . . . . . . . . . . . . . . . 137

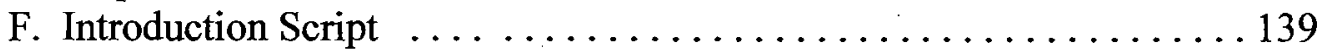

IRB Acceptance Form

VITA 


\section{LIST OF TABLES}

Table

Page

1. Means And Standard Deviations Of Dissociative Experience Scale Scores For Childhood Sexual Abuse By Family Conflict Analysis . . . . . . . . . . . . . .77

2. Means And Standard Deviations Of Global Severity Index Scores For Childhood Sexual Abuse By Family Conflict Analysis . . . . . . . . . . . 78

3. MANOVA Summary Childhood Sexual Abuse By Family Conflict . . . . . . 79

4. Means And Standard Deviations Of Dissociative Experience Scale Scores For Childhood Sexual Abuse By Family Expressiveness Analysis . . . . . . . .81

5. Means And Standard Deviations Of Global Severity Index Scores For Childhood Sexual Abuse By Family Expressiveness Analysis . . . . . . . . . . 82

6. MANOVA Summary Childhood Sexual Abuse by Family Expressiveness. . . . .85

7. Means And Standard Deviations Of Dissociative Experience Scale Scores For Childhood Sexual Abuse By Family Cohesiveness Analysis . . . . . . . 86

8. Means And Standard Deviations For Global Severity Index Scores For Childhood Sexual Abuse By Family Cohesiveness Analysis . . . . . . . . . . 87

9. MANOVA Summary Childhood Sexual Abuse By Family Cohesiveness . . . .88 


\section{CHAPTER ONE}

\section{INTRODUCTION}

As the 20th century ends and the 21 st century begins, researchers estimate that increased percentages of women will report that childhood sexual abuse (CSA) occurred at some point in their lifetime (Bryer, Nelson, Miller \& Krol, 1987; Chu \& Dill, 1990; Morrow \& Smith, 1995; Nishith, Mechanic \& Resick, 2000). Currently, reviews of scientific literature estimate that $20 \%$ to $40 \%$ of women in the general population, $24 \%$ to $44 \%$ of adult female outpatients and $40 \%$ to $60 \%$ of adult female inpatients claim a CSA history (Finklehor, Hotaling, Lewis \& Smith, 1990; Gold, Hill, Swingle \& Elfant, 1999; Swett \& Halpert, 1993).

Researchers also hypothesize that a significant number of female CSA survivors who experience multidimensional and debilitating symptoms may be unaware of treatment possibilities, unwilling to address trauma issues or, for varied reasons, unable to enter into treatment (Briere \& Elliot, 1993; Inderbitzn-Pisaruk Shawchuck \& Hoier, 1992). In addition, these untreated survivors are often members of under-researched populations such as female prisoners (Forward \& Buck, 1978; Gold, 2000).

Trauma theorists and researchers acknowledge that a CSA history often plays a crucial etiological role in triggering and maintaining a variety of psychological disorders across various domains of functioning (Briere \& Runtz, 1988; Browne \& Finklehor, 1986; Gold et al, 1999; Kessler \& Bieschke, 1999; Nishith et al., 2000; Terr, 1991). Dissociative disorders and Posttraumatic Stress Disorders (PTSD)—often part of the same CSA survivor profile-are the most frequently cited (Waites, 1993). Although CSA research has explored the coexistence of PTSD and other disorders such as Dissociative Disorders, 
little if any research has addressed the relationship between dissociation and psychological distress (Barret, Dadds, Dadds, \& Rapee, 1996; Gold et al, 1999).

A primary impetus for assessing the coexistence of dissociation and other symptom disorders is to determine whether such symptom clusters are part of the profiles of the most symptomatic CSA survivors. Kluft (1990) suggests dissociation is a maladaptive coping mechanism that disrupts the normal flow of consciousness, eliminating a sense of continuity in day-to-day living. Such disruptions often severely limit the CSA survivor's ability to interact with the world. While research examining which environmental and abuse variables determine the strongest dissociative responses (Collins \& Ffrench, 1998; Kluft, 1994) provides some clarification of etiologic origins, focusing only on dissociation ignores the possibility that other disorders or symptoms, coexistent with dissociation, may partially be responsible for the most symptomatic profiles.

Therefore, in order to assess what predicts the most symptomatic CSA population, logic demands that, in addition to dissociation, researchers should focus on mediators such as psychological distress, which affect individual functioning in ways similar to that of dissociation. Described not as discrete symptoms but as a diminished overall psychological functioning, psychological distress affects an individual's ability to participate in normal activities of daily life (Derogatis \& Lazarus, 1994). This description is very similar to the description of the impact of dissociation (Kluft, 1994, Liotti, 1992, Morgan, 1996).

If psychological distress and dissociation both are part of a survivor's profile, it is suggested that the persistent and combined effect of both dissociation and psychological distress creates an endless loop in which the heightened distress precipitates the need to 
dissociate and vice versa, Because untreated survivors may not possess emotional tools to interrupt or diminish this loop (Barrett, Dadd, Dadd \& Rapee, 1996), an untreated female prison population with histories of CSA would be expected to exhibit such multidimensional symptom presentations (Ursano \& Fullerton, 1999).

Numerous reasons exist to propel research towards clarifying the etiological origin of more symptomatic CSA presentations. Current clinical data shows that certain CSA survivors present with some of the most severe and multilayered pathology of all mental health clients (Nash, Hulsey, Sexton, Harralson \& Lambert, 1993). Theorists assert that adults with CSA histories, compared to non-CSA adults, report more global mental health issues, interpersonal problems (relationship or sexual concerns) and intrapersonal concerns (depression or anxiety). Those who study these resultant trauma responses also report that these same women often present with health concerns (eating disorders, alcohol or drug problems) and severe life disruption or life threatening concerns (dissociation and/or suicidiality) (Gold, Milan, Mayall \& Johnson, 1994; Waites, 1993).

Many of the above presentations of adult CSA survivors become entrenched by the CSA survivor's continually evolving patterns of extreme cognitive distortion and an inability to believe in the efficacy of his or her future (Collins \& Ffrench, 1998; Romans, Martin, Morris \& Herbison, 1999; Swett \& Halpert, 1993). Such beliefs, resultant of coexistent environmental influences during the abuse, are often carried into adult life (Koraleski \& Larson, 1997; Stuppy, 1996). Without clear data that explain and predict why certain individuals respond in more symptomatic ways, interventions may be ineffective in alleviating the impact of these and other symptoms on the adult CSA survivor's life (Courtois, 1988; Gold, 2000). Research must, therefore, continue to ferret 
out which variables initially influence the child CSA survivor's symptom response and determine whether these same variables are the etiologic origin of maladaptive adult responses.

Currently, a number of theories attempt to explain and predict why different CSA trauma responses develop. Kluft (1990), Koraleski and Larson (1997) and Morrow and Smith (1995) submit that many traumagenic factors are manifested before, during and after CSA occurs that determine the level of symptom development. These investigators note that such determining factors may be abuse-specific and/or environment related. Nash et al. (1993) theorize that the environment in which a highly symptomatic CSA victim lived was often more pathogenic than the abuse itself. By this, it is suggested the increased level of dissociation and multilayered symptomology result from the combined impact of dysfunctional family dynamics (precipitating residual effects even after the abuse stops) and CSA rather than CSA alone.

Specifically, some dissociation theorists cite dysfunctional family expressiveness, lack of cohesiveness and/or conflict (collectively identified for the purpose of this research as dysfunctional family dynamics) as primary mediators for level and type of dissociative response (Gold et al. 1999; Morrow \& Smith 1995). If dysfunctional family dynamics do represent one of the strongest mediating influences on dissociative magnitude, research utilizing female prisoners (who describe such dysfunctional family systems), could begin to identify the family's pathogenic influence.

Formative-year developmental theories are beginning to show a strong influence in current CSA research and the subsequent analysis of dysfunctional family dynamics' impact on dissociation (Koralelski \& Larson, 1997). Researchers and theorists suggest 
that CSA, especially if it occurs from soon after birth to seven years old, can hinder or diminish the child's normal developmental ability to attach, separate, emote, and believe in a safe world (Brock \& Mintz \& Good, 1997; Ford, Fisher \& Larson, 1997; Long \& Jackson, 1994; Nash et al., 1993).

Nigg, Westin, Gold and Silk (1992) and Koraleski and Larson (1997) note the importance of developmental theories in the elucidation of influences of dysfunctional family dynamics on increased trauma symptom responses. Most importantly, they suggest such theories, describing how a child should develop healthy constructs of experience and expression through their primary caretakers' emotional modeling, reinforcing, interpretation and labeling, can show how trauma disrupts these processes.

Most researchers agree that childhood sexual abuse emotionally impacts a child, regardless of when it occurs (Gold, 2000; Herman, 1992; Morrow \& Smith, 1995). Yet to fully understand how CSA affects an adult's mental health and coping abilities, it is important to look at the effect of CSA on the pre-development stage child (one who has yet to pass through normal emotional development) and the post development maladaptive stage (one who has passed chronologically through such stages, but due to the family environment, did not develop the expected emotional capabilities) (Bowlby, 1988; Collins, 1996; Gold et al., 1999). Children who have successfully moved through these developmental stages have developed traits such as healthy emotional expression, object permanence, self-soothing, and the ability to modulate or tolerate emotions. These traits in turn help the child develop a strong sense of safety, order and meaning, all which are necessary for individuation and healthy attachment (Bowlby, 1988).

CSA victims, who have yet to attain normal levels of emotional development 
(experiencing CSA before the normal development of personality and emotional expression), may have limited ability to understand or work through the emotional weight of the trauma (Barret, Dadds, Dadds \& Rapee, 1996). Skills are not yet acquired to modulate or tolerate the wide range of emotions associated with CSA. In addition, a predevelopment child, after the trauma, has no ability to attach to anyone in order to regain a sense of safety and trust. Without a sense of internal safety and trust, this type of CSA victim unsuccessfully continues to seek safety in the family. When the dysfunctional family does not provide safety or emotional comfort, dissociation, helping the child to separate from the intense emotions, may offer the only relief (Alexander \& Anderson, 1994; Waites, 1993).

The post development-maladaptive stage CSA victim can develop similar trauma reactions as the predevelopment CSA victim, not because of the age at which CSA occurred but because family dynamics hindered normal emotional development. Family relationships have already taught the post development CSA victim to not ask for or expect support. Subsequently, the shock and disruption of the CSA experience creates conflict between a victim's need for reassurance and safety and a self-protective acknowledgement that such reassurances will not come. This conflict and the lack of family support combined with the child's limited emotional resources often leaves the child isolated in his or her own fear (Herman, 1992). The fear is often compounded by an extreme sense of loneliness and hopelessness with dissociation as the only mechanism available for escaping these intense emotions (Liotti, 1992; Morrow \& Smith, 1995).

It appears that dysfunctional family dynamics, in general, limit the CSA victim's ability to intrapersonally and interpersonally deal with and process the trauma (Nash et al. 
1993). Research has shown that a major part of healing for any trauma survivor occurs as the victim expresses (as soon after the trauma as possible) and, with support, works through complicated trauma-related emotions (Boney-McCoy \& Finklehor, 1996; Brock et al., 1997). Those who haven't developed these skills may be destined to a protracted or incomplete healing process.

To further clarify how dysfunctional family dynamics affect differing CSA trauma responses, it is important to understand how the three mediating variables of cohesiveness, expressiveness, and conflict influence a child's coping mechanism (Haberstadt, Cassidy, Stifter, Parke \& Fox, 1995; Moos and Moos, 2002). The lack of family commitment, help, support and other isolating behaviors towards the child (lack of cohesiveness) compounds the CSA victim's diminished sense of safety and protection. The child's sense of threat, initially related to the abuse, most likely increases when the family does not provide sanctuary from, or emotional assistance with the processing of (expressiveness), associated fear or intense emotions (Heatherton \& Baumeister, 1991; Kessler \& Bieschke, 1999).

In addition, the CSA victim's lack of personal resources (in a family where the child wasn't taught nor allowed to express most emotions (expressiveness) limits the victim's ability to process and work through the trauma (Long \& Jackson, 1994). Functional families, with protective and nurturing influences, reduce the impact of ongoing stressors and often enhance the child's ability to seek non-family social resources (Moos \& Moos, 2002). Lastly, a conflictual family environment detracts from individual attention to the CSA victim's emotional needs. The family's own intense aggressiveness and anger (conflict) make it either unsafe or non-productive to express emotions, and therefore 
restrict the victim's healing (Finklehor et al., 1990; Ford et al., 1997).

The mechanisms of dissociation are closely associated with the emotional limitations that dysfunctional families impart upon a CSA victim. Theorists, as early as Janet (Kluft, 1990), proposed an innate ability of the mind to erect barriers of self-preservation against too much stimulation such as overwhelming emotions. Dissociation, a primary defense mechanism operating at all levels of consciousness, is one such barrier that is triggered for a CSA child victim within such a family (Braun, 1986; Briere \& Elliot, 1993). The inability to regulate or recognize feelings leaves the victim vulnerable to emotional overload from initial fear and shock as well as post-trauma reactions (Fredrickson, 1992; Gold, 2000; Waites, 1993; Zingnıan \& Boswell, 1996): The current research's premise is the etiologic base of most complex dissociative barriers is rooted in how dysfunctional family relationship dynamics initially activate dissociation.

Dissociation, as a response to the combined impact of CSA and dysfunctional family dynamics or to trauma alone, is described as an individual's experience of disruption to their usually integrated functions of consciousness, memory, identity and perception of the environment. These responses are not due to the direct physiological effects of a substance and are not indigenous to a particular location or culture (DSM IV, 1994). This altered state of consciousness (often described as trance-like) can occur both during and after the abuse. Dissociation provides a type of barrier from direct experience of the emotions, from the reality of the abuse and even from conscious interaction with others (Bloch, 1991; Collings, 1994; Collins \& Ffrench, 1998).

Waites (1993) suggests that dissociation is a creative coping response temporarily lessening the impact of both immediate and long-term effects of trauma. Nevertheless, 
dissociation can become maladaptive when it limits the individual's ability to interact with the world or to process emotions. As non-trauma based environmental triggers become associated with the trauma through sensory similarities the CSA survivor's need to dissociate increases, resulting in a continuing decrease in his or her sense of connectedness (Fredrickson, 1992). More specifically, certain noises, smells, tactile sensations and other sensory responses connect to conscious and unconscious memories of the traumatic event and through cued association triggers the dissociative response. The dissociative CSA survivor's already limited awareness of time, place, and self is further compounded by these reactions to environmental cues. Effects of this compounded dynamic produces wide-ranging and lingering effects for untreated CSA survivors long after the abuse has stopped (Terr, 1994; Williams, 1994).

A number of researchers propose that both environmentally-associated cues and the reactive numbness of dissociation continually activate anxiety and create a polarized existence of both hypervigilance and disconnection from the present environment (Cassiday, McNally \& Zeitlin, 1992; Everstine \& Everstine, 1993; Long \& Jackson, 1994; Van der Kolk, McFarlane \& Weisaeth, 1996). Rose (1996) concurs that the polarized existence of hyper-arousal and disconnectedness from the environment, living side by side with the impact of disrupted attachment, further increases the untreated survivor's need to dissociate. In addition, when dissociation creates a discontinuity of experience, the CSA survivor's already distorted perception of interpersonal and environmental interactions, becomes even more distorted, through lapses in time. This discontinuity in turns creates anxiety based in the individual's vulnerability to the environment. The heightened anxiety then reinforces a greater need to dissociate (Kluft, 
1990). This reciprocal symptom relationship, where the symptoms themselves continue to traumatize or destabilize the CSA victim, may well represent a primary influence for the most symptomatic CSA presentations.

Dissociation's protective function may insulate the CSA survivor so well that, as memories become less accessible, the individual may deny the reality of the abuse. The dysfunctional family relationship factor can once again compound the need for increased dissociation. More specifically, a CSA victim, even with repressed memories, has vague fears and emotions related to the abuse. When family members reinforce the denial of the abuse, this lack of support and under-lying unconscious fear of the abuse increases conscious fear and anxiety and thus dissociation (Ursano \& Fullerton, 1999). Even when the CSA survivor acknowledges the reality of the abuse, disbelief or denial within the family, still often results in the CSA victim's unresolved anxiety, fear and a sense of shame (Finklehor et al., 1990; Kluft, 1990). These conflicting realities create an even a greater need to detach (Fredrickson, 1992; Nash et al., 1993; Terr, 1991; Williams, 1994).

Although dissociation provides the survivor with tools with which he or she can separate from internal and external chaos, the mechanism does not appear to erase the impact of trauma. Researchers suggest that when CSA survivors are unable to maintain dissociative barriers (Cole-Detke \& Kobak, 1996; Heatherton \& Baumeister, 1991; Kluft, 1993), reactive responses, such as eating disorders, panic and anxiety disorders, longterm depression, drug and alcohol use and living high-risk lifestyles often emerge (Rosenstein \& Horowitz, 1996). Such responses have been described as having dissociative-like qualities and an ability to block introspective awareness (Mallinckrodt, 
McCreary \& Robertson, 1995; Rosenstein \& Horowitz, 1996; Swett \& Halpert, 1993;

Torem, 1986;Wilsnak, Vogeltanz, Klassen \& Harris, 1996)

With the numerous and problematic means by which dissociation can be triggered, maintained and compounded, psychological distress may be closely related to level of dissociative responses. Although logical deduction may lead to an assumption that a highly dissociative individual would minimally experience or be aware of anxiety and distress, it also seems reasonable to assume that the discontinuity of consciousness, produced by dissociative periods, would add to anxiety experienced before the dissociative episode. Even with such an expected relationship, minimal, if any research, has addressed whether psychological distress and dysfunctional family dynamics are related to higher levels of dissociation and if such a combination is manifested in the most pathological CSA symptom presentations (Heatherton \& Baumaster, 1991; Pianta Egeland \& Adam, 1996; Steinberg, Tobin \& Johnson, 1990; Waites, 1993).

If the current research finds that there is a significant relationship between CSA and dysfunctional family dynamics and levels of dissociation and psychological distress, replication studies could begin to confirm that such presentations are part of other highly symptomatic CSA survivor profiles (Ross, Anderson, Fleisher \& Norton, 1992; Spira, 1996; Swett \& Halpert, 1993). From such studies, comparison studies could assess whether these compounded responses manifest themselves for one CSA population and not another, while also identifying intervention needs for under-treated CSA populations.

Research of unaddressed CSA populations is extremely important to the continued development of comprehensive CSA theories. Researchers and clinicians have attempted to explicate the link between and within group similarities and differences of adult CSA 
survivors mainly through college and clinical populations. Gold (2000) and Morgan (1996) suggest that college samples, as a convenience population, show more resiliency than other CSA populations. They suggest that clinical populations are more likely to demonstrate higher levels of dissociation and distress than those of college samples but are more homogenous than the general population. These groups represent more polarized representations of CSA survivors (Rose, 1996). Zingman and Boswell, (1996) found that for college students acknowledging a CSA history and high levels of dissociation, dissociation, in general was not only associated with the dysfunctional low levels of family cohesiveness and expressiveness and high conflict, but also with high levels of family cohesiveness and expressiveness. For most clinical populations, high levels of dissociation are related to only low levels of family cohesiveness and expressiveness (Gold, 2000).

In order to gain a comprehensive understanding of how a CSA victim's trauma response influences subsequent adult personality development, beliefs, behaviors and psychological functioning, trauma researchers must be able to access CSA populations, who, along with varied symptom profiles, have more diverse ethnic and demographic profiles than the clinical or college populations (Morgan, 1996). This research's implementation was partially based on the premise that the use of diverse CSA populations can begin to identify more specific between and within group differences of larger samplings of CSA survivors than has been done in previous research. This then could create a stronger arguement to either generalize theories to other CSA populations or develop new population specific theories. (Briere \& Elliot, 1993; Inderbitzen-Pisaruk, et al. 1992: Speigal, 1986; Williams, 1994). 
Researchers note that the last 10 years have brought a rapid increase in the number of women incarcerated in US prisons (Wilsnack et al., 1996). Henderson, Schaeffer and Brown (1995) suggest that compared to the general population, incarcerated women have higher rates of CSA history and of most mental disorders. Such statistics seem logical when etiologic studies of offending behavior are reviewed. Allen, Hauser and BormanSpurrel (1996) and Farrington (1995), in their surveys of female prisoners and general populations, found that individuals who experienced either CSA or maltreatment during childhood are significantly more likely to have had a history of problem behaviors during adolescence that have been associated with adult offending behavior. These can be, but are not limited to serious and violent delinquency, teen pregnancy, high school dropout rates and higher incidences of mental health problems. In conjunction with such findings, reviews of literature show that female deviancy, when compared to male deviancy, has historically been more often linked to mental illness or moral deficiency (Baunach, 1988; Farrington, 1995; Sweezy, 1998). Yet, few developmental studies have compared female prisoners to non-prisoners to assess why some CSA survivors manifest symptoms and behaviors differently than other victims (Spurrel \& McFarlane, 1996).

Surveys, such as those by Gorsuch (1998), show that even with documented statistics of mental health issues for incarcerated women, few in-depth mental health or trauma specific interventions are offered to these individuals. The correction system's male oriented, mental health services provided to females are very limited in their ability to treat these highly symptomatic women. Gorsuch suggests that programs designed to "fix" male and female prisoners often do not reflect an understanding of the complexity of problems. Many female prisoners with extreme mental health issues are described as 
"hard to place" within the prison system and are constantly moved between prisons rather than treated. When released, these women often return to society with unaddressed and unaltered mental illness (Farrington, 1995; Gorsuch, 1998; Greenfield \& Minor-Harper, 1991; McCellan, Farabee \& Crouch, 1997).

As described earlier, many of the normal developmental processes are delayed or disrupted by the interaction of CSA and dysfunctional family relationships (Ford et al., 1997; Kessler \& Bieske, 1999, Nash et al., 1993). Demographic statistics from studies of offending behavior, found a high percentage of incarcerated women with families that were restrictive in expression, lacked cohesion and experienced high conflict. These same studies show high percentages of CSA histories and multigenerational patterns of abuse and incarceration (Burkett, 1991; Sweezy, 1998). Even so, there does not appear to be any research that investigates the relationship between a CSA history and family dynamics on an incarcerated female's symptom presentation.

Given the high rates of CSA histories, high levels of mental health issues, and high levels of family dysfunction within this highly symptomatic but under-served and underresearched CSA population, it is surprising that the prison population has not been utilized to help elucidate the etiologic origin of some of the most pathogenic CSA presentations (Morgan, 1996). It is also relevant that prison surveys illustrate that the cycle of incarceration, as with abuse, has been passed down through many generations (Greenfield \& Minor-Harper, 1991). Thus, research on the etiologic origin of incarcerated female CSA survivors may not only help emphasize the unaddressed mental health issues and data by which to design more effective interventions (Sweezy, 1998) but could also affect future generations. 
In summary, a wealth of data have been described that suggests some type of relationship exists between CSA and the dysfunctional family dynamics of expressiveness, cohesiveness, and conflict and that this relationship influences levels of dissociation and psychological distress. Data were presented that described possible reciprocal relationships between dissociation and psychological distress. Yet there is little research that verifies what determines the most entrenched levels of dissociation. It is suggested that the co-existence of psychological distress may be part of such symptom clusters and that research of the highly symptomatic yet untreated female prisoner population may begin to clarify such questions.

\section{Research Problem}

The present study of incarcerated females was designed to determine whether, for this population, a relationship exists between the combined influence of a remembered CSA history and perceived dysfunctional family relationship dynamics and resultant symptom magnitude. Specifically, the goal of this research is to determine what, if any relationship exists between the independent variables of a remembered CSA history and perceived family dynamics of expressiveness, cohesiveness and/ or conflict (Moos \& Moos, 1986) and magnitude of dissociation and level of psychological distress (Derogatis, 1975).

The proposed study will utilize the female prison population, often described as having high levels of mental illness, of being less resilient, and of being under-researched and under-treated (Henderson et al., 1995). In order to clarify which factors are consistent over different CSA populations and which dynamics are population specific, utilizing this highly symptomatic, less resilient, and culturally under-researched CSA population is of extreme importance. Significant findings from this and similar studies could be compared 
to findings from studies of convenience populations (whose findings are limited in their ability to be generalized) (Gold et al., 2000). Furthermore, whereas past research has focused on discovering the etiological base of dissociation or specific psychological symptoms, little research has addressed the relationship between or coexistence of dissociation and other symptom clusters such as psychological distress. Research that expands our understanding of other co-existent factors within the trauma response, may help predict what are the most significant predictors factors for the most symptomatic CSA presentations.

\section{Significance of Study}

Childhood Sexual Abuse (CSA) and Dissociation research, investigating how and why different trauma responses develop, continues to advance theoretical understanding of both group and individual differences in CSA survivors. Yet the majority of CSA and dissociation research utilizes college and clinical populations, both of which may be more polarized extreme representations of CSA victims. It has been suggested that many CSA victims exist who are not members of either of these populations (Gold et al., 2000) and to whom findings cannot be generalized.

Historically, incarcerated women are reported as having both extensive histories of CSA and a high prevalence of mental health issues (Rose, 1996), and yet are underrepresented in CSA research. Literature reviews suggest that the majority of studies related to female prisoners focus on the occurrence of CSA or mental health issues while ignoring the etiology of such symptoms (Greenfield \& Minor-Harper, 1991; Gorsuch, 1998).

The present study could provide more robust findings related to a number of domains 
and populations. Significant results would support present theoretical assertions regarding the etiologic base of dissociation, which have been confirmed with convenience populations. With the more diverse prison population, this data could begin to suggest whether the above is true or not across cultural populations such as in African American, Hispanic, and Native American cultures. In addition, significant findings confirming the influence of the three dysfunctional family dynamics on more symptomatic responses of dissociation and psychological distress, would suggest that other under-researched CSA populations whose family dynamics mirror this sample should be assessed.

Clarification of the influences of family on multidimensional symptoms is also specifically relevant to the female prison population. Literature reviews show that more than $60 \%$ of incarcerated women acknowledge a history of CSA. This same group of women also describe dysfunctional and non-supportive family histories and generational histories of abuse (Farrington, 1995; Henderson et al., 1995). Even so, there appears to be very few mental health intervention strategies with symptom-specific orientation for these women (Greenfield \& Minor-Harper, 1991). Such data can be important for longreaching intervention development such as therapeutic and psycho-educational programs that both educate this population on the effect of CSA and family dynamics and help individuals work through unresolved emotions. Insight gained from such interventions could initiate change in the structure of the women's core beliefs impacted by generational patterns of abuse and incarceration, may help reduce recidivism rates for this population (Farrington, 1995) and could break the described dysfunctional cycles for future generations (Baunach, 1982; Gorsuch, 1995).

Furthermore, this investigation, by utilizing an under-researched population, continues 
the historical growth pattern of trauma research. Early researchers investigated the trauma response of World War II and Vietnam War veterans. These studies, in turn, provided a theoretical base from which new theories, related to childhood trauma and adult trauma and, (in later years), community disasters, were developed and tested (Everstine \& Everstine, 1993; 1989; Sutker \& Allain, 1995; Terr, 1991; Walker, 1991; Williams, 1994). Under-researched and under-treated populations such as incarcerated female CSA survivors are the new frontiers for CSA researchers. This new phase of trauma research can begin to expand clinicians' and researchers' understanding of trauma and its complicated impact on an individual life.

In summary, this study has both theoretical and practical applications for both general and specific CSA populations. If findings confirm that female prisoners make up a highly symptomatic CSA population and that family dynamics influence such presentations, it is imperative that studies be replicated with other under-treated and under-researched populations. Even more so, such findings would emphasize the need for development of more effective interventions for this population and the general highly symptomatic CSA survivor population (Collins, 1994; Gold, 2000).

\section{Assumptions}

Following is a list of assumptions related to this study.

1) Female inmates will answer questions related to nuclear family dynamics and the occurrence of sexual abuse in a truthful manner in relation to current perceptions of childhood family history.

2) For those claiming a childhood sexual abuse history, it is assumed that they have some type of memory of the abuse. 
3) For those not identifying a childhood sexual abuse history, it is assumed that this is due to the lack of occurrence rather than loss of memory of the event.

4) Participants understand that prison officials have no access to the confidential research information.

5) Participants will not feel coerced into participation in this research project.

6) The nature of the questions on the Background Information Sheet and the Family Environment Scale are retrospective in nature but do accurately measure the constructs intended to be measured.

7) Participants have at least an 8th grade reading level.

\section{Limitations}

While certain assumptions have been made that would appear to balance out certain limitations, realistically there are limitations associated with this study that could contradict the above assumptions. Self-selected population samples can produce biased results if individual motivation for participation does not also provide motivation to fully attend to the research protocol. The reward and punishment structure that is often the base of a prison system (Gorsuch, 1998) may, through the inmate's imagined benefit or loss in their relationships with staff, influence them to attend but not fully participate. When recognition for participation is a primary motive, an individual may attempt to "just get the project done" without attention to detail. Such limited participation is further compounded by confidentiality rules that participants are not identified on their answer sheets. Participants could then complete answer sheets in a haphazard way without fear of negative impact.

Self-report measures, similar to the four used in this design are based on the assumption 
that participants will answer the questions in a truthful manner. Historically such measures have been shown to be sensitive to the respondent's emotional state and environmental factors. In conjunction with this, inmates either attempting to "look good" or "look bad" may answer questions based on this bias rather than what may be known to be true. Thus, the "truthful" assumption may be suspect. The "faking bad" can be a cry for help. Instructions given to participants regarding mental health service referrals after the session for those distressed by topics of the questionnaires, request breaking confidentiality so that issues can be assed by the counselor. Such "faking bad" profiles may be completed on the assumption that increased symptomology will enhance referral chances. Individuals "faking good" may be worried about breaches of confidentiality within the prison system and will not trust the statements of the administrator.

The sensitive nature of certain questions related to sexual abuse and other traumatic memories might hinder some participants from answering the questions truthfully. Participants will be reminded that they can leave the administration session at any time. A large $\mathrm{N}$ could protect against such an effect. This research is not expected to have a large enough number of participants of any cultural group to be able to generalize findings yet historically, research reviews indicate that certain cultural representations are under-researched (Gold, 2000). Thus, with the acknowledged under-representation in CSA research of ethnic groups such as African American, Hispanic or Native Americans could make this study's findings more valuable to CSA research. It is hoped that further studies of clinical and control populations of a similar ethnic mix will attempt to replicate significant findings.

In relation to the specific study of dissociation, some individuals, especially those 
experiencing high levels of dissociation, may not be able to recall certain feelings or memories of the CSA experience or of their families (Everstine \& Everstine, 1993; Harvey \& Herman, 1994; Kluft, 1990). In general, it is hoped by the design of the study and through a large population sampling that these limitations will be minimal in their influence.

\section{Definition of Terms}

1) Childhood sexual abuse (CSA) - CSA is defined as sexual behaviors initiated by an adult toward a child. Childhood, for purpose of this research, is defined as 18 years and younger (utilizing cut off age of past studies; Bryer et al., 1987; Chu \& Dill, 1990). The sexual interaction with the child may have been obtained through coercion, threatened or actual physical harm or through misinformation (Dutton, 1994). CSA involves, but is not limited to: the adult touching or fondling any body part of the child, making sexual remarks that are inappropriate and may initiate uncomfortable feelings for the child, exposing the child to any type of sexual material, attempting and/or completing oral or anal penetration of the child's genitals with any part of the adult's body or any type of object and other situations where the child is exposed to sexual acts occurring between others (Everstine \& Everstine, 1993; Fredrickson, 1992; Inderbitzen-Pisaruk, Shawchuck \& Hoier, 1992; Waites, 1996). The Background Information Sheet will include this definition.

2) Dissociation - Dissociation, for the purpose of this research, is defined as an altered state of mind with varying levels of consciousness that occurs on a continuum from brief lapses in awareness to extensive periods such as days, weeks, or months. Dissociation involves a disruption in the usually integrated functions of consciousness, memory, 
identity or perception of the environment (DSM-V, 1984). The present study will use the Dissociative Experience Scale - DES (Carlson \& Putnam, 1993) for measuring dissociation. Items on the DES address both non-symptomatic dissociation and pathological dissociation.

Analysis of results will assess individual and mean DES scores as being in a high, medium and low level of dissociation. The low levels of dissociation (DES scores from 0-20) are experiences described as losing slight awareness of time and place. This can occur when an individual is absorbed in an activity such as watching television, driving a car or other interactions that create a slight hypnotic or trance like state. This level of dissociation often does not have its origin in trauma.

Medium levels of dissociation (DES scores 20-30) are described as experiences more disruptive to everyday consciousness and are often a symptom response to a traumatic experience. These dissociative states are typified by the following experiences, not all of which occur together, but often do: minor problems with access to long and short term memory; a sense of unfamiliarity in a place that should be familiar; feeling of being enveloped in fog; feeling a discontinuity of place and time, sometimes accompanied by a sense of fainthess without loss of balance; and other experiences of time or memory loss.

The highest levels of dissociation (DES scores $>30$ ) are described as states of mind that involved greater and more intense problems with all of the above levels in addition to extensive forgetfulness of names, faces and familiar environments. Some of these higher levels of dissociation can be manifested by a sense of separation of one's personality into distinct different feeling parts, commonly known as Dissociative Identity Disorder (Chu \& Dill, 1990; Collins \& Ffrench, 1998; Gold et al., 1999; Greaves, 1993; Kluft, 1990). 
3) Emotional Abuse - In order to separate sexual abuse effects from emotional abuse, emotional abuse, described on the Background Information Sheet, will describe emotional abuse as both verbal and nonverbal forms of coercion and/or isolation behaviors (Dutton, 1994). For the purpose of this research, this will include: the action of an adult, adults or an older child toward a child who is 18 years or younger and who is at least five years younger than the abusive older child is. Behaviors could include but were not limited to the individual communicating toward the child in a demeaning manner (often expressed through unwarranted responses), giving continual criticism to the child's behavior without little or no basis in fact, communicating through verbal threats, both public and private intimidation and humiliation to the point that it reinforces the child's behaviors that reactivate the abuser's behavior.

4) Global Psychological Distress (For the purpose of this research GSI is interchanged as a measure of Global Psychological Distress). This term is defined as the overall psychological distress indicated by the Global Severity Index (GSI) of the SCL-90-R. This scale is designed to be a summary of total amount of distress experienced by the individual. GSI scores are calculated by adding the nine primary symptom dimensions scores. The primary symptom dimension scores are resultant of specific type of responses to each symptom group. The GSI score is based on the assumption that higher scores reflect that the individual's responses to questions on each separate scale indicated that he or she was extremely or quite a bit distressed about issues the question addressed. Global Severity Scores above 63 are described as indicative that certain psychological disorders might exist and denote further clinical analysis (Derogatis \& Lazarus, 1994).

5) Incarceration - This is defined as any form of legal restriction of an individual within 
a confined environment. This restriction will have occurred through the due process of the legal system.

6) Perceived Family Cohesiveness - This is defined by the scale of similar name on the Family Environment Scale (FES - Moos and Moos, 2002). Cohesiveness is described by the authors as the degree of commitment, help and support that the individual feels that his or her family members provided for one another. Levels of cohesiveness, for purpose of analysis, will be broken down into low, medium, and high levels based on means and standard deviations for normal and distressed populations. (Moos \& Moos, 2002). 7) Perceived Family Conflict - This is defined by the scale of similar name on the FES (Moos and Moos, 2002). The authors describe conflict as the amount of openly expressed anger, aggression and conflict the individual feels his or her family members expressed. Levels of conflict, for purpose of analysis, will be broken down into low, medium, and high levels based on means and standard deviations for normal and distressed populations. (Moos \& Moos, 2002).

8) Perceived Family Expressiveness - This is defined by a scale of a similar name on the FES (Moos \& Moos, 2002). Expressiveness is described by the authors as the degree or extent that an individual describes family members are or were allowed to act openly and express feelings directly to each other. Levels of expressiveness, for purpose of analysis, will be broken down into low, medium, and high levels based on means and standard deviations for normal and distressed populations. (Moos \& Moos, 2002).

9) Perpetrator/Abuser - This is defined as the person inflicting the physical, emotional, or sexual abuse against a child.

10) Physical Abuse (PA) - In order to separate sexual abuse effects from physical abuse, 
physical abuse is defined on the Background Information Sheet as any behavior that involves the intentional use of force in such a way that there is risk of death, physical injury, harm or pain regardless of whether the behavior actually results in such (Dutton, 1994). Physical abuse can include but is not limited to the following behaviors of the adult toward a child: shaking; hitting; burning; choking; forcefully holding or restraining the individual; forcing the individual to take an unwanted substance; use of lethal weapons and/or other verbal or threatening actions that may stop short of body contact but influence the actions of the victim.

\section{Research Questions}

For incarcerated women, the following research questions will be asked:

1) What is the relationship of the independent variables of a remembered childhood sexual abuse (CSA) history and levels of perceived childhood family conflict (CON-FES scores) with the dependant variables of dissociative magnitude (DES scores) and levels of psychological distress (GSI SCL-90-R scores)?

2) What is the relationship of the independent variables of a remembered childhood sexual abuse (CSA) history and levels of perceived childhood family expressiveness (EX-FES scores) with the dependant variables of dissociative magnitude (DES scores) and levels of psychological distress (GSI-SCL-90-R scores)?

3) What is the relationship of the independent variables of a remembered childhood sexual abuse (CSA) history and levels of perceived childhood family cohesiveness (COH-FES scores) with the dependant variables of dissociative magnitude (DES scores) and levels of psychological distress (GSI-SCL-90-R scores)? 


\section{Hypotheses}

Hypothesis I: There will be a significant relationship between a remembered CSA history and perceived childhood family conflict with magnitude of dissociation and level of psychological distress.

Hypothesis II: There will be a significant relationship between a remembered CSA history and perceived childhood family expressiveness with magnitude of dissociation and level of psychological distress.

Hypothesis III: There will be a significant relationship between a remembered CSA history and perceived childhood family cohesiveness with magnitude of dissociation and level of psychological distress. 


\section{CHAPTER TWO}

\section{LITERATURE REVIEW}

Reviews of research over the past 20 years indicate that childhood sexual abuse (CSA) of female children is very prevalent and that as adults, many of these survivors suffer from pervasive psychological problems (Roth, Newman, Pelcovitz, van der Kolk \& Mandel, 1997). Data from such investigations estimate that $20 \%$ to $60 \%$ of American women experience sexual abuse before age 18 (Barko-Cello, 2001; Briere \& Elliot; 1993; Brock et al., 1997; Gold, 2000). Other studies verify CSA's crucial etiological role in the development and persistence of some of the most acute and multi-layered pathology of women seeking or participating in therapy (Chu \& Dill, 1990; Nishith et al., 2000, Waites, 1993).

Demographic analysis from numerous researchers (Alexander \& Anderson, 1994; Collings, 1994; Gold et al., 1999;Williams, 1994; reviewed demographic data from recent CSA studies and found certain consistent factors in the profiles of a significant number of adult CSA survivors: dissociative difficulties, severely diminished psychological functioning, high levels of psychological distress, and life-long histories of social and professional debilitating mental health issues. Similar reviews show that in addition to dissociative disorders, the most frequently documented cluster of severe pathology found for these survivors is Post Traumatic Stress Disorder, in which dissociative-like symptoms form part of the diagnostic profile (Briere \& Elliot, 1993; Everstine \& Everstine, 1993; Fredrickson, 1992).

CSA theorists, researchers, and clinicians have recently turned their investigation towards clarifying the etiologic origin of dissociation (Collins \& Ffrench, 1998; Ellason 
\& Ross, 1995; Greaves, 1993; van der Kolk, van der Hart \& Marmar, 1994). Specifically, notable interest has been shown towards analyzing how and what mediating variables impact both the development and severity of dissociation (Gold, 2000). Dissociation occupies this center of attention due to its unique and often contradictory properties of both protecting an individual from the onslaught of emotional distress while at the same time, compounding and exacerbating the emotional dilemma.

Dissociation initially develops as a coping mechanism that helps protect the individual from immediate emotional effects of the trauma (Kessler \& Beischke, 1999; Ross et al., 1992; Ursano \& Fullerton, 1999). At the same time, dissociation, with its ability to block immediate awareness, causes a disruption of the normally integrated functions of consciousness, memory, identity, and perception of the environment (DSM IV, 1994; Finklehor et al., 1990; Nash et al., 1993; Spiegal, 1986; van der Kolk et al., 1994). CSA survivors, as research participants, describe how their dissociative symptoms leave them feeling as if their lives are separate from the everyday environment and pervasively disconnected from the reality of the abuse (Fredrickson, 1992, Kluft, 1990; Stuppy, 1996; Swett \& Halpert, 1993).

Although CSA researchers, such as those listed above, concur with basic descriptions of dissociation, disagreements exist as to whether CSA alone affects pathological dissociation or whether mediating factors play a more crucial role (Brock et al., 1997; Finkelhor et al., 1990). Most studies of dissociation appear to be split between two schools of thought: those who suggest that selected characteristics of the abuse, such as penetration, level of violence, number of times that the abuse occurred, etc., generate the strongest impact on dissociation; and those who suggest that environmental variables 
such as early family relationship dynamics may be more pathogenic in their interaction with the abuse than the abuse itself (Bryer, et al., 1987; Gold, 2000; Liotti, 1992; Nishith et al., 2000).

Research exploring the impact of family functioning on a CSA survivor's resiliency or maladaptive responses has begun to determine which types of family functioning better account for the most complex and pathological dissociative presentations of survivors (Brock et al., 1997). Yet, such research has not analyzed how family functioning impacts other symptom development and severity level in relation to their co-existence with dissociation or if the co- morbid existence further complicates the dissociative response. Psychological distress, often the result of numerous symptoms manifesting themselves together, appears to have similar etiologic origins to dissociation (Barret et al., 1996; Macleod \& Bryne, 1996; Rosenstein \& Horowitz, 1996). Such a coexistence of dissociation and psychological distress may predict the most symptomatic CSA population.

To better understand how such coexistence could occur, it is important to explore how researchers describe the influence of dysfunctional family dynamics on the survivor's trauma response. Initial symptom development is also significantly associated with the victim's lack of coping skills required to deal with the overwhelming emotions related to their abuse (Haberstadt et al., 1995). Such a lack of adaptive skills for individuals, separate from abuse, has been associated with dysfunctional families, which do not provide adequate acceptance and/or modeling of emotional expression (Nash et al., 1993).

Brock et al. (1997) utilized regression analysis of multiple CSA studies to confirm that 
family functioning better predicts levels of symptom pathology than does sexual abuse status. Brock et al. found that functional families, or at least the perception of such, serve as a protective factor with respect to psychological symptomology. These researchers found that individuals who describe a CSA history and dysfunctional family relationships had the highest level of psychological distress when compared to the CSA / Functional family relationship, no-CSA/ dysfunctional family relationship and the no-CSA/ functional family relationship groups. Lower distress levels were found for women of CSA/ functional family relationship than those with CSA/ dysfunctional family relationship. No significant difference was found for stress levels of the CSA/ functional family relationship and no-CSA/functional family relationship. Brock et al., note that family, while impacting pathology can also impart a resiliency factor (as seen in the lower distress levels for those with CSA and functional family systems).

The impact of the family environment on childhood development has not only been the focus of CSA research. Developmental studies also find that children with dysfunctional family relationships and all types of abuse may experience extensive problems in the course of adolescent development. The variety of negative outcomes can include but are not limited to delinquency, early pregnancy, alcohol and drug abuse, and emotional mental health problems (Gorsuch, 1998; Stuppy, 1996). Such findings appear to be consistent with prison studies showing that anywhere form 20 to $40 \%$ of incarcerated women identify histories of dysfunctional and non-supportive families (Farringtion, 1995; Greenfield \& Minor-Harper, 1991).

It should be alarming to CSA researchers that demographic prison research show high percentages of incarcerated women with histories of CSA and mental health problems 
(with minimal exposure to any forms of mental health interventions), yet are not part of most CSA research populations. This participant pool provided data from a different type of population rather than college or clinical. It provided information on CSA survivors who, on cultural and income level status, may not be part of the convenience populations.

Research on incarcerated females is not only important in relation to identifying untreated CSA populations but is important to CSA theory development and applied interventions. There is a major problem in being able to generalize findings from current CSA and dissociation studies of convenience populations to the more robust population of CSA survivors. Under-researched CSA populations, such as incarcerated females, must be identified and analyzed to discover what theories can be generalized across populations and which are population specific.

This study's premise was based on the historical pattern of trauma research. Through more than two-thirds of the twentieth century, trauma theory advanced when spurred on by the needs of a new trauma population. Early trauma studies from the 1930's to the 1950's investigated trauma responses of World War I and II veterans. From such studies, the Shell shock theory was developed as an explanation of veterans' post-war diminished ability to function (Sutker \& Allain, 1995). The next thirty years researchers extrapolated evidence from a loosely defined shell shock theory and developed the basis for the diagnosis of Post Traumatic Stress Disorder (PTSD) (Everstine \& Everstine, 1993).

As the PTSD diagnosis began to be defined in the 60 's and 70s, so did research on the etiological base of the different symptoms of the diagnosis, especially dissociation. At the same time, the growing public awareness of the impact of childhood abuse resulted in clinical environments seeing growing numbers of CSA survivors. Trauma research once 
again expanded as researchers utilized knowledge from treatment of Viet Nam Veterans' PTSD, to develop general theories about trauma and in the long run, to CSA (Sutker \& Allian, 1995; Terr, 1994; Waites, 1993).

Studies show that an undetermined number of highly symptomatic CSA survivors may be unwilling to come into treatment, may be unaware of the origin of their problems, or may be living in environments where treatment is not offered (Farrington, 1995; Gorsuch, 1998; Gold, 2000; Inderbitzin-Pursaruk et al., 1992). Incarcerated women, described as a highly symptomatic and having extensive histories of CSA, by current environment alone, are part of these untreated populations (Farrington, 1995). As a population often associated with numerous dysfunctional family relationship factors, female prisoners with a CSA history also appear to be vulnerable to the most symptomatic trauma responses (Boney-McCoy \& Finklehor, 1996; Gorsuch, 1998; Sweezy, 1998).

\section{The Impact of Sexual Trauma}

Reviews of trauma literature suggest that sexual abuse of female children is, at any one time, experienced by up to $45 \%$ percent of the population before the age of 18 years old and contributes to many adult psychological problems (Everill \& Waller, 1994; Finklehor et al., 1990; Heatherton \& Beaumeister, 1991; Mallinckrodt et al., 1995; McClellan et al., 1993; Steinberg et al., 1990). Certain factors, however, differentiate the CSA trauma response from other responses to traumatic events.

Morrow and Smith (1995) suggest that CSA clients and research subjects often describe general trauma reactions of fear, sudden psychological pain and immediate disruption of daily life activities, as do victims of wars, natural disasters and community violence, yet also found that certain descriptors were specific to CSA survivors. Yet, for 
CSA survivors, along with the feelings of powerlessness and helplessness, appear to experience a more extreme sense of loss of self-identity endured long after the cessation of the CSA. CSA survivors also describe life long patterns of disrupted emotional attachments, an inability to relate to others, and long-term, often lifelong, hyper-vigilance related to continual experiencing of flashbacks, sensitivity to environmental triggers and disruptive dissociative tendencies (Briere \& Elliot, 1993; Kluft, 1993; Swett \& Halpert, 1993).

Briere and Runtz (1988), examining a non-clinical sample of women with a CSA history compared to non abused women, found higher levels of acute and chronic dissociation along with greater levels of depression, somatization and anxiety for the CSA women. The most significant associations, that of parental incest and duration of the abuse were related to higher levels of dissociation, led these researchers to assert that family relationship factors had significant impact.

Briere and Runtz further suggest that more severe levels of dissociation may evolve due to the exploitation of "love" and "trust." Not only does the individual's response to the horror of CSA impact the need to dissociate, but so might his or her frustrated and unresolved natural need for comfort from a non-supportive and "dangerous" family. Such a family influences a different type of response than to a one-time traumatic incident and/or when a CSA victim is immediately protected and comforted by family members.

Nash et al. (1993) in a survey of clinical observations of CSA clients, state that many therapists concur that the context in which the CSA occurs, is the most powerful group of intervening variables affecting symptom development. Thus, by such a hypothesis, highly symptomatic CSA survivors are significantly more likely to have endured the abuse 
within a complex environment of family dysfunction. It may well be that the powerlessness and lack of control endured within the experience of CSA, may be mirrored by the dysfunctional family system. This sense of continued threat may increase the need to dissociate.

In support of the long-term effects of CSA, Lenore Terr (1991), extrapolating evidence from numerous studies of PTSD, suggests that long term abuse, if not processed emotionally (i.e. that the survivor's surrounding environment restricts such processes) can create profound character changes. These long-term changes often involve an inability to identify feelings, inaccessible rage and sadness, massive denial of the abuse and often repression of memory of the events. It appears such an environment may have impacted the adult victim, who describes herself as highly dissociative, where cognitive disengagement was the only release from these unprocessed emotions. Researchers such as Kluft (1994), Sandberg et al., (1994) and Browne and Finklehor (1986) also found that the most intense forms of dissociation are significantly associated with individuals who identify themselves as victims of long-term abusive relationships as well as CSA. Such long-term abusive relationships, in their replication of the dysfunctional family dynamics during the CSA survivor's childhood, impact the greater need to dissociate.

Dissociation does not only disrupt an individual's life but research indicates there are a number of factors related to dissociation that can put the CSA survivor at risk. Analysis of past research shows that, not only will anywhere from $25 \%$ to $40 \%$ of women in American society experience CSA, but that these same women are more likely than nonvictims to be sexually assaulted in adulthood (Nishith et al., 2000; Sandberg et al., 1994; Swett \& Halpert, 1995). Kessler and Bieschke's (1999) retrospective study on shame and 
revictimization also suggest that those with CSA histories and dissociative symptoms have a greater risk of adult revictimization. Their investigation found that shame and associated negative self-attributions appear to put the adult victim more at risk when dissociation desensitizes or blocks the individual's awareness of danger. The shame will also cloud the CSA victim's awareness of being at risk by discounting his or her own internal sense of safety. These studies suggest that there are not only psychological, interpersonal and intrapersonal problems arising from CSA, but also possible continued exposure to danger.

Even with the alarming statistics related to number of victims and the multitude of effects resultant from CSA, current statistics may be under-estimated (Bryer et al., 1987; Everill \& Waller, 1994; Gold, 2000). If there are individuals or even cultural groups who feel counseling and or/disclosure are unneeded or unavailable, if they don't attend colleges, then there is a likely chance that these individuals may be part of underresearched CSA populations.

In conjunction with these untapped research resources, general research-based knowledge of the impact of CSA alone does not adequately describe or predict why certain survivors are more symptomatic than others. To determine if population estimates are limited and/or what dual symptom presentations are most prevalent, CSA trauma responses must be looked at across different populations. Use of the under-researched, culturally different incarcerated CSA population, can begin to clarify such issues.

The Impact of Dissociation

Dissociation is described as one of the primary psychological defenses used to protect against the extreme emotional impact of trauma. This psychological defense is an 
unconscious mechanism that manifests itself in dramatic and often pathological ways, impacting an individual's experience of both self and the world (Kluft, 1993; Van der Kolk et al., 1994). Dissociative symptoms are often seen as part of the presentation of Posttraumatic Stress Disorder (PTSD; DSM IV, 1994) clients, even though dissociation is not listed as one of the primary symptoms of PTSD. In addition, research suggests that, even if different types of trauma precipitate different organizations of symptom clusters, dissociation appears to be one traumatic response that individuals experience across many different trauma-related disorders (Barker-Cello, 2001; Briere \& Elliot, 1993; Brock et al., 1997) and consistently higher, and more debilitating levels of dissociation have been associated with the prolonged experience of CSA (Chu \& Dill, 1990; Harvey \& Herman, 1994; Kluft, 1990).

Many factors influencing the development of many symptoms and levels of an individual's PTSD response, similarly affect the dissociative response. Dissociation is defined as one of the most immediate and characteristic symptom responses following exposure to extreme stressors of a traumatic event. PTSD is defined as the development of a group of characteristic symptoms in response to the overwhelming stressors of a traumatic event. PTSD occurs after exposure to either a direct personal traumatic experience or witnessing the event, both which involve actual or threatened death or serious injury or threat to one's physical integrity (DSM IV, 1994). These events include but are not limited to; all forms of abuse, extreme personal loss, war, earthquakes or other natural disasters or man-made events such as the Oklahoma City Bombing in 1995. The individual's response to the trauma is often emotionally laden with intense fear, hopelessness, helplessness, anxiety, and horror. 
Dissociation, through a numbing of general responsiveness, is also a reaction to the overwhelming feelings of loss of control, fear, and other emotions activated by the traumatic experience (Liotti, 1992; Morow \& Smith, 1995). This numbing helps to maintain a persistent avoidance (as described in the DSM IV, 1984) of stimuli associated with the event. This can include but is not limited to: persistent re-experiencing of the event, increased arousal, sleeplessness, and highly sensitive startle responses (Bloch, 1991; Collins \& Ffrench, 1998; Ross et al., 1992; Speigal, 1986). These stimuli become constant reminders of the experience and reactivate all the associated emotions.

Many current CSA researchers concur with the definition of dissociation as a traumatic response, involving a breakdown in the typical correspondence between and/or within the three modes of cognitive responses, motor responses, and physiological responses (Bernstein \& Putnam, 1986; Chu \& Dill, 1990; Kluft, 1992). The essential features of dissociation are disruptions in the usually integrated functions of consciousness, memory, identity and/or perception of the environment. This lack of association can be sudden, gradual, transient, or chronic (DSM IV, 1994).

Spira (1996) describes the escape process of dissociation as a suspension of awareness of current time, logic, space, and other self-distinction such as one's thoughts, emotions, sensations, behaviors, and at times consciousness. Dissociation initially helps the individual escape an unbearable situation through such suspension. Further more, Spira proposes that this response is resultant of two types of coping: repression (out of dissociative consciousness) and division (within levels of dissociative consciousness).

In addition, dissociation is described as both a normal and psycho-physiological mechanism that is both a normal response and a component of a number of different 
mental health disorders (van der Kolk et al., 1994) from mild depersonalization, anxiety disorders to Dissociative Identity Disorder (DSM IV, 1984). Many dissociative symptoms are also included in criteria for other disorders such as Acute Stress Disorder, PTSD and Somatization disorder (DSM IV, 1994). Not all of these disorders are associated with CSA in the DSM- IV descriptions (DSM IV, 1994).

The majority of the human population experiences low levels of dissociation. These common experiences are described as; spacing out, losing time while driving or brief daydreaming in which one feels slightly disconnected from time and space (Chu \& Dill, 1990). On the other hand, medium levels of dissociation are often a more pathological symptom response to a traumatic experience and can be disruptive to everyday consciousness. These dissociative states are typified by the following experiences, (not all of which always occur together): minor problems with access to long and short term memory; a sense of unfamiliarity in a place that should be familiar; feeling of being enveloped in fog; feeling a discontinuity of place and time, (sometimes accompanied by a sense of faintness without loss of balance); and other experiences of time or memory loss.

As pathological responses to extreme trauma, high levels of dissociation involve more intense and enduring manifestations of lost periods of time, forgetfulness of names, faces and familiar environments and an inability to remember both recent and past events. The most acute experience of the highest level of dissociation falls under the diagnosis of Dissociative Identity Disorder (DID) (DSM IV, 1994: Kluft, 1990; DSM IV, 1994; Speigal, 1986). DID is described as an organization of personality in which the individual has two or more personality states that appear to take control of behavior. Each state may have its own relatively enduring patterns of perceiving, relating to, and thinking about the 
environment and self. These personality states may or may not be aware of other existing personalities (Spira, 1996).

Dissociation researchers suggest (Briere \& Elliot, 1993; Gold et al., 1999; Kluft, 1992) that, dissociation, regardless of severity level is initially a creative way to deal with overwhelming stimuli. As a primary adaptive coping strategy, dissociation reduces tension and defends against any type of increased arousal or unbearable emotions precipitated by trauma or the trauma related cues. While dissociation provides some protection against such stimuli, this experience is not continual. Thus, the inability to constantly maintain such barriers leaves the victim susceptible to further exposure to trauma-related cues. What initially is a rational and protective way to keep immediate and long-term effects of the trauma separate from the victim's conscious awareness ends up re-victimizing the individual through its disengaging properties (Dutton, 1994; Morrow \& Smith, 1995, Stuppy, 1996).

In other words, dissociation co-existing with unresolved trauma issues can influence an ongoing dichotomy of hyper-arousal and intrusions, living side by side with disengagement from the environment (Harvey \& Herman, 1994; Malinsky-Rummel \& Hoier, 1992). One of the ways this process occurs is when dissociation's disruptive tendencies are affected by, and act in concert with, trauma-based flashbacks. A flashback triggered by trauma-related environmental cues transports a trauma victim, through physical and emotional memory back to the traumatic event. At times when a victim is more consciously aware, the visual and other trauma related sensory memories initiate heightened anxiety and fear through partial or full replication of the initial traumatic event. Dissociation's coping mechanism (Chu \& Dill, 1990; Ursano \& Fullerton, 1999) is 
reactivated to protect the individual from the anxiety, but, when the dissociative period ends, anxiety returns. Thus, until desensitization to the cues occurs, the victim remains in this co-existent cycle (Malinosky-Rummel \& Hoier, 1992).

Waites (1993), in reviews of trauma research, also agrees that dissociation has creative coping properties that become maladaptive. She suggests that dissociation's most damaging impact is its' disruption to an individual's conscious ability to attend to cognitive and sensory input. She notes that, most importantly, dissociation separates an individual from the internal self and diminishes his or her ability to protect from revictimization (Kessler \& Bieschke, 1999; Kluft, 1990). This separation from self can also be disruptive to the CSA survivors' healing process. One of the hardest tasks of the healing for the CSA survivor is gaining subjective and conscious sense of control over, thus access to, emotions previously protected by dissociation.

Finklehor et al. (1990) and Nash et al. (1993) suggest that, along with inanimate environmental cues, differing family environments can either detract or add to the need to dissociate. As noted earlier (Impact of Sexual Trauma section) dissociation researchers find that for those who experience CSA in the first seven years of life, who claim little family support or help in dealing with the overwhelming trauma-related emotions, that interrupted attachment and individuation processes and are related to highest levels of dissociation when compared to CSA survivors with more family support (Ford et al., 1997; Kluft 1990). It is suggested that the attachment process, where individuals develop a sense of safety, trust and self-identity through connection with their primary caretaker (Bowlby, 1988), when interrupted, leaves the chifd CSA victim witf an inability to tolerate or modulate emotions. For a child with no sense of security, dissociation 
becomes the only form of distancing from the emotions and unsupportive family environment (Haberstadt et al., 1995). In conjunction with these factors, the disruptive attachment and non-supportive family are not the only phenomena of family relationship dynamics that play a role in triggering dissociation. Research shows high levels of dissociation to be related to unsuccessful attempts to disclose about the abuse (Fredrickson, 1992; Terr, 1994).

Dissociation has been associated with both co-varying and co-existing symptomology. Many women who initially present with eating disorder or drug addiction problems may later disclose dissociative reactions (Rybici, Lepowsky \& Ardnt, 1989; Cole-Detke \& Kobak, 1996). Some researchers hypothesize that an inability to maintain dissociation, may impact the emergence of an eating disorder or addiction in order to replace dissociation's numbing effects (Heatherton \& Beaumeister, 1991; Mallinckrodt et al., 1995). Research investigating the relationship between trauma histories and eating disorders, has begun to explore whether individuals utilize behaviors such bulimia, anorexia and even self-mutilation (triggering an opiate-like response) to either distance from or regulate the overwhelming trauma-related emotions as well as a grounding technique from dissociation (Steinberg et al., 1990; Torem, 1986).

Throughout this section, strong support has been noted for manner in which both environmental cues and family relationship dynamics variables play a major role in initiating and/or maintaining dissociation. Even so, the following section explores how family relationship dynamics can have an over-arching effect across all other environmental variables and the resultant level of dissociation. 
The Impact of Family Relationship Dynamics

It has been suggested that family environment and family members' adaptation mutually influence each other (Moos, Finnet \& Cronkite, 1990). Healthy adult personality characteristics affect the quality of family relationships, individual goals, and the family as a cohesive unit (Moos \& Moos, 2002). Moos and Moos note, for example, that a healthy family environment -- one that allows and models healthy emotional expression (expressiveness) and also provides support for each other during a crisis, creates a stronger family structure because individual emotional strength. A family that provides a stable base built on trust, responsiveness, and identification with the family as a whole (cohesiveness) instills individuality in its members and each individual responsiveness influences even more trust within and outside of the family. A family that has normal" levels of confrontation-- (conflict)-- reduce ongoing stressors by allowing expressions of disagreement and enables expression of divergent views without threat. All of these dynamics enhance not only individual and family support resources, but also enhance the survivor's ability to seek assistance from external social resources (Nash et al., 1993; Pianta et al., 1996). Polarized dysfunctional levels of the above family relationship dynamics severely limit coping abilities of family members, especially in developing children. An adult with behavioral or emotional problems, more often than not, will affect individual members' coping abilities as well as the self-esteem and psychological adjustment of any child within that family (Ford et al., 1997; Moos \& Moos, 2002, Pianta et al., 1996).

To better understand the impact of family relationship dynamics on trauma responses Wilson (1994) proposes that research based on integrative theories provides the most 
comprehensive method by which to describe, explain and predict the ways that traumatic events, within the context of family and other social structures, systematically impacts age-related levels of emotional development and psychological functioning. Wilson suggests that understanding how genetic factors and family affect developing personality, coping styles and development of self-identity, researchers can more readily address the multiple influences on trauma responses. More specifically, an integrative theory looks at individual capacities that normally evolve during developmental years of childhood (within the influences of the family or caretaker, biology and/or the environment), the manner in which these processes may have been interrupted, limited or completely obstructed and how these altered processes interact with the abuse. What is important to note in Wilson's proposal, is that each CSA victim's trauma response is affected by multi-dimensional influences. Bowlby (1988) theorized that attachment, one of the primary influences, is any form of a person's behavior in which he or she tries to obtain or maintain proximity to another individual, whom he or she perceives as better able to cope with the world. This individual history of interaction with attachment figures often determines systematic differences in how the individual copes with stress-related cues (Pianta et al., 1996).

Developmental and attachment theorists, years before trauma theorists, hypothesized that dysfunctional family relationships during the child's developmental years often result in pathological behavior (Collins, 1996; Haberstadt et al., 1995; Pianta et al., 1996). Specifically for a young CSA victim, the combination of constant vigilance against future abuse and a lack of family support pull energy from the developmental tasks of individuation and separation. Lacking any concrete self-identity, the CSA victim lives in 
constant turmoil over instinctually seeking, but never finding comfort and closeness from the abusive family. Gold (2000) and Sandberg et al., (1994) agree that the dysfunctional family's inability to provide emotional modeling and a safe environment results in the CSA victim's inability to attach and develop emotional coping skills. They further suggest that such inability on the behalf of the CSA victim results in intolerable, unending, and unresolved loneliness that only dissociation may have the ability to abate.

CSA research has begun to investigate the predictive ability of such variables such as successful or unsuccessful attachment to primary caretaker(s) and constructs such as safety and trust (Bowlby, 1988; Ford et al., 1997). Rosenstein \& Horowitz, 1996) investigating the impact of the primary caregiver's attachment style on the child's attachment processes and possible development of pathology, found that normal emotional growth is severely limited by the caregiver's own maladaptive or disrupted attachment processes. In addition, they found that insecure attachment organization of the child was significantly associated with unresponsive, interfering, rejecting and otherwise insensitive parenting. For a child experiencing loss and/or trauma, this type of insecure and non-comforting relationship with the caregiver, and family as a whole, is a mediator of long-term pathological effects into adulthood and has been noted as an "at risk" factor for both child and adult pathology (Gold, 2000). Pianta et al., (1996) found, in their investigation of attachment and self-reported psychiatric symptoms on the MMPI II, that $85 \%$ of the women who were categorized as having a pre-occupied style of attachment had the highest scores of pathology. These same women had also identified unresolved experiences of loss or trauma, experienced during childhood, as enduring into adulthood.

While successful attachment prior to abuse can predict more effective coping skills, the 
enduring aspects of the family environment affect the victim's interaction with the world past the chronological developmental stages and the abuse itself (Allen et al., 1996). This wide ranging influence of the family is partially due to the family environment being where the child learns the rules of accepted emotional expression, where the child forms values and core beliefs and where these determinants influence levels of resiliency or maladaptive coping (Finklehor et al., 1990). Without effective coping skills or belief in one's own right to exist, the CSA victim is often unable to tolerate or modulate their trauma related emotions, becomes overwhelmed and hopeless and will often seek any way (often maladaptive) to distance from these stressors; one such response being dissociation (Finklehor et al., 1990).

Nash et al., (1993), analyzing the relationship between adult pathology and CSA, also found that CSA survivors who identified families with high levels of conflict (a family relationship dynamic), high levels of boundary confusion (either high or low family relationship dynamic of cohesiveness), and high levels of behavior rigidity (low family relationship dynamic of expressiveness) had significantly higher levels of dissociation and other pathology than those with CSA and medium levels of the three noted family relationship dynamics. He notes that polarized aspects of these family relationship dynamics create intolerable levels of emotions and unresolved loneliness that often triggers higher levels of dissociation.

Bryer et al. (1987) in studies of CSA survivors also found that levels of dissociation and multi-layered symptomology more often than not result from the dysfunctional family system and the family members' response to the abuse than from the CSA alone. Specifically, they found that family expressiveness, cohesiveness, and conflict constitute 
determinants of the magnitude of dissociative responses. Bryer et al. and other researchers find the lack of expressiveness, an extremely enmeshed or disconnected family system (extremely low or high cohesiveness) and a family experiencing ongoing high levels of conflict, impact higher levels of dissociation than CSA alone (Moos \& Moos, 2002; Morrow \& Smith, 1995; Nash et al., 1993; Zingman \& Boswell, 1996). In conjunction with the above findings Brock et al., (1997), examining differences among abused women from functional and dysfunctional families and non-abused women from the similar family types found that the abused group identifying dysfunctional families reported the highest levels of psychological distress when compared to other family types. No difference was found between the abused/functional family group and the non-abused/functional family group. Brock et al., also found that during initial therapeutic intervention, the dysfunctional family group of survivors who endorsed chronic PTSD symptoms also identified profoundly limited psychosocial functioning, Axis II characterological impairment (personality characteristics) and severe problems with regulation of affect, consciousness and bodily functioning. This may partially describe how dissociation, an affect regulator, may continually be called upon and become maladaptive in adult years

Ford et al., (1997) also found that chronic PTSD patients with impaired object relations (one important, unimpaired part of self-identity development) experience significantly more chronic trauma symptoms (including dissociation) and are more impaired psycho socially than those without impaired object relations. They suggest that impaired object relations, represented by the individual's lack of self identity, lack of security in relationships and an inability to emote, is an end product of non-responsive, non 
supportive and punitive caretakers. The consequence of such impairment is a malevolent world construct that continues to limit the victim's coping abilities or the ability to seek support beyond family.

Not only do some survivors interpret the world as unchanging and malevolent but the dysfunctional family relationship dynamics also affect the individual's self-attribution of blame (Briere \& Elliot, 1993; Nash et al., 1993). For example, Kessler and Bieschke (1999) found that negative attributions based in participant's described non-supportive family interactions contribute to high levels of shame and that shame was a statistically significant predictor for revictimization of adult female CSA survivors.

Although many of the studies in this chapter cite dysfunctional family relationships as one source of higher levels of dissociation for both CSA victims (Gold, 2000), few have fully analyzed what, if any symptoms are consistently co-morbid with dissociation, how family relationship dynamics affect these co-morbid diagnoses and whether there is a reciprocal intensity relationship between dissociation and the co-existent symptom. Some symptoms or symptom clusters that have been described as having a partial etiologic base in trauma include, but are not limited to, eating disorders, PTSD, self-mutilation, anxiety disorders and somatization disorders. Many of these disorders also have been shown to have an etiologic base in dysfunctional family interaction and an inability to emote (Steinberg et al, 1990; Root; 1991).

A small number of studies have investigated the co-occurrence of eating disorders and incest. Mallinckrodt et al. (1995) noted that research participants who endorsed both severe abuse and emotionally restrictive family dynamics identified extremely limited introspective awareness (the ability to perceive and label emotional stress and well 
being). Noted earlier (Steinberg et al., 1990), eating disorders facilitate a detachment from unresolved and intolerable emotions in ways similar to dissociation. It would seem to follow then, that many of the dynamics that affect greater needs to dissociate, would influence stronger association with the eating disorder. It appears that dysfunctional family relationship dynamics are one of the strongest influences of eating disorder development (Steinberg et al., 1990). Even so it appears that no research ahs identified a consistent co-morbid diagnosis of dissociation and any form of eating disorder, why only a subdivision of CSA survivors develop an eating disorder along with dissociation and why others only develop an eating disorder.

Certain symptomatic personality presentations have been associated with a CSA history. Nash et al. (1993) suggest that the etiologic base of a Borderline Personality Disorder (DSM IV, 1994) diagnosis lies within the conflict between an instinctual need (from birth on) for emotional support and expression and a dysfunctional family system that blocks resolution and satisfaction of these needs. This type of unresolved dichotomy was previously described (Sandberg et al., 1994) as a precipitator of increased need to dissociate. Once again, although some individuals with a Borderline Personality Disorder diagnoses, identify a CSA history, this is not true of all individuals with this diagnosis. Even so, certain dysfunctional family relationship dynamics appear to be primary predictors for the development of a Borderline Personality Disorder.

Increased levels of psychological distress (an indicator of the presence of numerous psychological symptoms that are emotionally destabilizing) have also been associated with an inability to tolerate or modulate emotions (Derogatis \& Lazarus, 1994) and as a primary symptom of chronic PTSD (Ehler et al., 2000). AS noted earlier, the inability to 
tolerate and modulate emotions is often due to lack of family responsiveness and emotional modeling (Pianta et al., 1996). While dysfunctional family relationship dynamics seem to influence higher levels of psychological distress and psychological distress is noted as a primary symptom of PTSD (DSM V, 1994), it appears that no CSA research has looked at the possibility that, for the majority of symptomatic CSA survivors, psychological distress may be co-morbid with dissociation.

In conjunction with the limited research of co-morbid diagnoses, especially psychological distress, it also appears that many of the dysfunctional family relationship dynamics associated with higher levels of dissociation and psychological distress are associated with predicting offending behavior. As noted earlier, incarcerated females, as a population are described often psychologically symptomatic yet untreated and as having a high percentage of CSA histories (Farrington, 1995). Investigation of the co-morbid existence of dissociation and psychological distress could provide more clear and explicit data for development of intervention programs that begin to diminish mental health problems of female prisoners with CSA histories but also the most symptomatic CSA survivors.

\section{The Impact of Psychological Distress}

As noted previously, psychological distress is defined as an indicator of the presence of numerous, emotionally destabilizing, psychological symptoms (Derogatus \& Lazarus, 1994). Such destabilization is often influenced and represented by unresolved emotional issues, projected negative or anxiety-based cognitive constructs and thinking as well as an inability to tolerate or modulate the issue-related emotions (O'connor \& O'conner, 2003). It appears that researchers investigating the etiologic origin of psychological 
distress find that dysfunctional family relationship dynamics related to expressiveness, conflict and cohesiveness have an escalating influence on the distress and anxiety similar to the way these family relationship dynamics influence higher levels of dissociation (Brock et al., 1997).

For example, Barret et al., (1996) in a controlled study of family treatment of childhood anxiety, report that a large percentage of their parent participants, who describe themselves as anxious adults, report origination of anxiety problems in childhood. Barret et al. found that the participant's children also endorsed high levels of anxiety in response to their parents' negative feedback and emotional and verbal restriction. This group of parents, identifying their own dysfunctional parenting styles, reported these styles evocative of their own parents. Thus for at least two generations the dysfunctional family relationship dynamics of negativity and restrictiveness predicted high levels of anxiety in subsequent generations. Barret et al. and other researchers provide the base for future research to investigate the impact of family on a variety of symptom presentations beyond that of trauma responses (Kemp \& Neimeyer, 1999; Nash et al., 1993). Findings reported by Barret et al. (1996) suggest that, due to similar dysfunctional family relationship dynamics influencing dissociation and psychological distress, the co-morbid diagnosis of dissociation and psychological distress should exist within the most chronic and symptomatic CSA presentations.

There could be a number of reasons that more pathological CSA trauma responses are based in a co-existence of dissociation and psychological distress. As noted by Harvey and Herman (1994), Malinsky-Rummel and Hoier (1992) and other dissociation researchers, the lack of coping skills needed to tolerate or modulate psychological 
distress impacts a greater need to dissociate (Baumeister, 1991; Kessler \& Bieschke, 1999). When a dissociative period ends, distress from lost awareness as well as renewed sensitivity to the trauma cues increases levels of distress. This in turn creates renewed need to dissociate (van der Kolk et al., 1996). It is also suggested that awareness of lost time during dissociation would lessen the survivor's sense of safety and/or belief in being able to maintain constant vigilance against further abuse. The diminished sense of safety in turn increases anxiety, which in turn reactivates dissociation. Without mental health intervention or development of emotional skills by which to interrupt the circular process of distress and dissociation, the individual may remain caught between the two responses. If such a relationship does occur, then untreated CSA survivors with both high levels of dissociation and psychological distress may represent the most debilitated CSA population.

To gain a better understanding of why such a relationship could develop, it is important to understand how attachment difficulties increase psychological distress. Kemp and Neimeyer (1999), investigating the manner in which interpersonal attachment styles of a general population of women affect individual experience of psychological distress, found that women identifying preoccupied attachment styles, were significantly more likely to have more intrusive mental health symptoms, thus, according to the definition of psychological distress, higher levels of psychological distress when compared to women with adaptive attachment styles. This was found to be especially true after stressful events like family fights, loss of job, etc. Logical deduction would assume that a child with a preoccupied attachment style would be expected to have higher levels of psychological distress after the CSA (a stressful event) occurs. This is based on the 
assumption that a non-supportive, non-emotive family and conflictual family, which limits a child's ability to developing effective coping mechanisms (Nash et al., 1993) would precipitate continual stressful events even after the victim has experienced CSA.

Research investigating resiliency factors also supports the premise that psychological distress, like dissociation, is strongly impacted by personality and attachment styles originating in the childhood influence of caretakers. Ehler, Boos and Maecker (2000) investigating resiliency in response to trauma found that mental defeat was not significantly associated with the stressor severity of a traumatic event. They suggest that cognitive constructs formed during the childhood such as a sense of controllability or lack of, and the individual's coping strategies are significant predictors of levels of mental defeat and that mental defeat has its etiologic origins in disrupted states of emotional development. Individuals, whose core beliefs include mental defeat, often present as highly anxious about negative outcomes. Specifically they found that, for political prisoners living with extreme restrictions and in traumatic environments, the stressor level of the event was not a significant predictor for high levels of mental defeat. Ehler et al. (2000) found that prisoners with a strong sense of self and coping skills did not have high levels of mental defeat, even though they experienced the same political imprisonment as those with extensive thoughts of mental defeat. They did find that mental defeat was a unique predictor of PTSD symptoms, of which anxiety is a primary symptom. It is further suggested that an individual's defeatist attitude plays a part in the prediction of failure, diminished belief that support will be offered if difficulties occur as well as influencing hypervigilance towards such failure.

Mental defeat could also be closely related to dissociation. Part of the enduring 
dissociative response of CSA survivors has been associated to unresolved safety issues (Ursano \& Fullerton, 1999). If the survivor, through dysfunctional family dynamics, develops a pathological process of worry and negative future-oriented attributions, then one could assume that safety issues, as a child and as an adult, are never resolved. The survivor would continually find need to dissociate as he or she lived within constant hypervigilance towards future harm, distress over these expectations and over unresolved trauma issues (Ehler et al., 2000).

The influences of cognitive constructs of mental defeat and negative future cognitions on a CSA survivor's level of psychological distress are closely aligned. For example, Pianta et al., (1996) and MacLeod and Bryne (1996) found that anxious and mixed anxious/ depressed individuals generate more enduring beliefs in future negative experiences than control groups. Both groups of researchers, although acknowledging research is limited in determining what factors of negative thinking predict negative results, hypothesize that anxiety, based in long-term negative thinking and predictions, influences behavioral responses that affect negative outcomes. Specifically, they suggest that fear-related current and future-oriented cognitions, (impacted by one's early life experiences), result in heightened anxiety about interactions with the surrounding environment. Such anxiety is often accompanied by hopelessness and worry that deters the individual from attempting or successfully completing goals (Ehler et al., 2000). It would appear that this anxiety and belief structure would then precipitate anxiety about future endeavors. This pattern would most likely transfer over to the ability to deal with trauma related issues and symptoms.

If high levels of anxiety have a partial etiologic base in negative-oriented thinking, 
and such dynamics have an etiologic origin in dysfunctional family relationship dynamics, this could explain how the reciprocal and confounding relationship of dissociation and psychological distress make up the most complex and enduring CSA symptom profiles. For example a CSA victim living within a dysfunctional family with low expressiveness and cohesion and high conflict, who is not allowed emotional exploration of his or her reaction to the abuse, and who is thwarted from disclosing or receives negative responses, could form negative beliefs about his or her safety or healing from the abuse. Such an environment would influence heightened distress related to fears of future abuse, the psychological dilemmas he or she may be experiencing and the lack of support. Such an environment has been described as precipitating the need to dissociate (Waites, 1993). A return to more conscious awareness brings all the unresolved issues described above to the individual's awareness. Such a pattern could be part of an enduring loop between dissociation and psychological distress.

Research such as Ruscio, Borkavec and Ruscio (2000) also support the above hypothesis of a co-morbid existence of dissociation and psychological distress. Investigating the latent structure of worry in normal and pathological presentations of clients, Ruscio et al. found that CSA survivors endorsing excessive levels of worry and psychological distress (usually associated with the Generalized Anxiety Disorder GAD diagnosis), spend more time engaged in worrying about threatening events than CSA survivors with "normal" levels of worry. Psychological distress's association with mood disturbances is supported in Coyne and Schwenk's (1997) findings, in their investigation of resiliency against mental health problems, that, for non-psychiatric medical patients, the appearance of distress without a significant mood disturbance is associated with little 
or no symptomology or any impairment of role functioning. For psychiatric populations, though, psychological distress is a strong indicator of the presence of mood disturbances and impairment of functioning. Interestingly, these researchers found that for most college populations, (often described as made up of mostly resilient individuals - Gold, 2000) psychological distress is not associated with mood disturbances.

The research described in this section suggests that dysfunctional family relationship dynamics affect the development of psychological distress or beliefs and attitudes that themselves initiate high levels of distress (Coyne and Schwenk, 1997; Ehler et al., 2000; Pianta et al., 1996; Ruscio et al., 2000; Waites, 1993). The impact of these dynamics may also explain why incarcerated females are expected to present with a combination of high levels of dissociation and psychological distress. As an untreated CSA survivor, incarcerated females, already identified with histories of dysfunctional family relationship dynamics (Farrington, 1995) similar to those associated with worry and negative thinking, in all probability, have developed pathological processes of worry and negative future oriented attributions. At the same time, similar family relationship dynamics have been described, for CSA survivors, as the etiologic origin of high levels of dissociation. Thus the previously discusses complicated ways that dysfunctional family relationship dynamics and disrupted attachment affect coping skills and resiliency against high levels of dissociation and now, psychological distress, seem closely aligned with family histories and coping abilities of female prisoners.

\section{Women in Prison}

As noted previously, researchers estimate that for incarcerated women, occurrence of sexual abuse before the age of 18 years old ranges from $41 \%$ to $85 \%$ (Dekleyn, 1996; 
Greenfield \& Minor-Harper, 1991). Kurshan (1997) notes that the prevalence of CSA histories is, for most researched offender populations, greater than for general researched populations and that many members of these same prison populations endorse long-term psychological problems without any form of psychiatric intervention (Greenfield \& Minor-Harper, 1995; Henderson et al., 1995; Rose, 1996). Incarcerated females also often report multi-generational abuse, partner abuse, rape, and re-victimization in prison (Greenfield \& Minor-Harper, 1991; Harvey \& Herman, 1994). Such correctional studies also suggest that a large percentage of family members of the female prisoners have also been in jail, while $11 \%$ of these women have at least a second-generation family member who had been to prison. With such high rates of trauma histories, unresolved emotional issues, and generation patterns of offending behaviors, it is also alarming that the female prison population continues to enlarge. Statistical reports (U.S. Bureau of Justice Statistics, 2003) show that the female prison population increased by $75 \%$ between 1986 and 1991. Other estimates suggest that in a nine-year-period from 1980 until 1989, the national female prison population grew by 27,000 (Greenfield \& Minor-Harper, 1995). The female state and federal prison population increased 4.9\% from December 31, 2001 to December 31, 2002 with the current female prison population at around 97, 491 individuals. Male prison populations only grew $2.4 \%$ during this same period in $2001-$ 2002 (United States Bureau of Justice Statistics, 2003).

The current female prison demographics show $6.6 \%$ of sentenced offenders in state prisons are females with $64 \%$ of these females from non-white ethnic groups (of whom many members are not the typical mental health service consumers nor part of CSA research. In that such statistics probably denote that there is a proportionate increase in 
the number of untreated CSA survivors within US prisons, it is surprising that incarcerated women's mental health issues and CSA histories are under-researched.

A logical deduction from the above statistics is that this population has a great need for mental health intervention, especially in the area of unresolved trauma. Yet to design more effective interventions, this under-researched population's (with possible different dynamics than other CSA research groups), emotional profiles and CSA histories must be analyzed to better understand the etiologic origin of their symptoms. At the same time, as noted in previous sections, offending behavior is predicted by similar family relationship dynamics to that of the etiologic origin of higher rates of dissociation and psychological distress of CSA survivors (Morrow \& Smith, 1995; Nigg et al., 1992). It may well be that there are both similarities and differences between incarcerated CSA survivors and other CSA populations. Research of incarcerated females could also help differentiate these issues.

One area of research with prisoners that parallels research on the etiologic origin of CSA trauma symptoms is the investigation of ways attachment processes and family relationship dynamics predict offending behavior. DeKlyen's (1996) study of disruptive behavior disorders and intergenerational attachment, found that the mixture of insecure child attachment and insecure maternal attachment, influenced by the parent's maladaptive relationship with his or her parents, had a significant impact on the child's continued disruptive behavior and on the parent's limited ability to effect change.

DeKlyen suggests that psychodynamic research, along with offender research that describes relationships between behavior disorders, and future offending behavior explains the origin of many generational patterns of incarcerated females. Historically, 
theories of general psychodynamics and attachment theorists show that replication of parenting styles is related to internal representations of intimate relationships formed during early childhood and later transferred to adult relationships (Bowlby, 1988; Ehler et al., 2000; Pianta et al., 1996; Wilson, 1994). McCellan et al., (1997) and Farrrington (1995), in research of the childhood etiological base of offending and antisocial behavior, find for individuals with extensive criminal histories after age 18, histories of poor parental relationships including lack of parental support, parents with incarceration histories and experiences of either direct violence or observed parental violence or conflict. These same participants also described an inability to emote and a lifelong sense of abandonment.

Other research on the relationship between attachment issues and coping abilities appear closely aligned with how family relationship dynamics influence offending behaviors. Allen et al., (1996), investigating the relationship between lack of resolution of trauma with primary caretakers and emotional difficulties in adulthood, propose that insecure attachments are linked to difficulties ranging from depression to severe problem behaviors. For upper-middle class adolescents hospitalized at age 14 years old, lack of resolution of previous trauma with primary attachment figures was significantly associated to periods of hospitalization and accounted for much of their insecure adult attachment when re-interviewed 11 years later when compared to similar sociodemographically non-clinical high school students. Moreover, insecure adult attachment organization of these same individuals at age 25 was also linked to self-reported criminal behavior and use of hard drugs in adulthood (Henderson et al., 1995). In relation to general CSA populations, clinical studies show higher rates of drug/alcohol use in 
treatment samples of adult CSA survivors than fro treatment samples of non-abused women (Briere \& Elliot, 1993).

It is interesting to note that dysfunctional family relationship dynamics related to cohesiveness', expressiveness', and conflict's impact on CSA symptom development (Nash et al., 1993) are addressed in offender research. Rose (1996) in a comparison study of minimum security female inmates and college students found for female inmates more ethnic diversity, lower levels of education completion, higher rates of CSA histories and more isolated lifestyles. For these same groups of offenders family variables associated with their history of delinquency included parental criminality, cruelty, passive or neglectful parenting (low expressiveness and low cohesiveness), erratic or harsh parenting (high conflict). CSA, developmental and attachment research has shown that the lack of positive connections with family members is related to disruptive biases and distorted-destructive thinking in interpersonal relationships (Gold et al., 2000; Kessler \& Breschke, 1999; Mallinkrodt et al., 1995).

It is not surprising then that Henderson et al. (1995) found higher rates of personality disorders for female offenders, already noted as having higher rates of CSA histories (McCellan et al., 1997) than for women in the general population. Interpersonal problems and coping deficits affected by lack of positive family relationships not only impact the ability to work through trauma issues (Kemp \& Neimeyer, 1999) but also is associated with repetition of offending behaviors. Sweezy (1998), in a study of incarcerated women with histories of recidivism, asserts that those prisoners who endorsed non- supportive families, high levels of guilt and unresolved mental health issues had recidivated more than prisoners who identified supportive families. 
It has been noted that lack of intervention, combined with a victim's continued immersion within an abusive environment affects increased anxiety and a greater need to dissociate (Ehler et al., 2000; Pianta et al., 1996; Ruscio et al., 2000). Once again, it is a logical deduction that incarcerated and untreated female CSA survivors would be highly symptomatic. Henderson et al., (1998) and Gorsuch (1998) identify female prisons replicating abusive home environments. Incarcerated women are often watched by a majority of male guards and have little privacy or sense of boundaries. Staff enforced punitive routines and their paternalistic attitudes often mimic non-expressive and high conflict families of many symptomatic CSA survivors (Nash et al., 1993). Female prisoner's described continued sense of lack of safety, and boundary violation (either low or high cohesive family dynamics) would, for these individuals, create a continued sense of violation, threat of future abuse and an inability to work through trauma issues. It is suggested that for the incarcerated female CSA survivor, the prison environment would influence a continued need to dissociate and hypervigilant sensitivity to environmental triggers (Henderson et al., 1995).

With the continued increase of female prisoners -- more than 78,000 women in U.S. Prisons in 1997 to 97, 491 female prisoners in 2002 (US Bureau of Justice, 2003) -- the associated demands for how to address the multi-dimensional issues of both their mental health and their general rehabilitation proportionally increases. At the same time in 1998, more than $70 \%$ of female inmates were single parents (Bureau of Justice Statistics, 2003). These statistics, along with research describing generational histories of maladaptive parenting, incarceration and abuse for female prisoners (Farrington, 1995) confirm the need for analysis of the etiologic origin of female prisoners' symptom presentation and 
whether such presentations are related to the combined impact of dysfunctional family relationships dynamics and occurrence of CSA becomes even more crucial. With such a large population of single parents, the described generational dysfunctions could be passed to the next generation. Information gathered from this study thus, may not only help to inform and educate the women as to how their lives have been affected, but may also make them more aware of how their children can be affected by the same dynamics.

In conjunction with the impact research of offending women could have on dysfunctional generational patterns and untreated mental health issues it is important to note that studies of incarcerated females and reviews of offender research show that prison populations include a high percentage of African Americans, Hispanics and single parents, cultures who members are often not part of the mental health consumer or general CSA research population (Banauch, Satkoloft \& Bowman, 1982; Gorsuch, 1998). Effective interventions as well as replication studies designed from significant findings on the relationship between CSA and dysfunctional family relationship dynamics may begin to address the multi-layered issues previously ignored by mental health providers and researchers.

In conclusion, it appears that there are many similarities between the ways that CSA and dysfunctional family relationship dynamics affect higher levels of dissociation and psychological distress as well as an individual's coping abilities. Similar dysfunctional family dynamics are also noted as impacting the female prisoner's struggle with mental health issues. Thus the etiologic origin of dissociation, psychological distress, and offending behavior, all appear rooted in the ways that family diminishes or limits a child's normal developmental process. At the same time, dissociation and psychological 
distress appear closely related in their response to the environment and to trauma, thus signifying a possibility of a reciprocal relation if present in the same symptom profile. It seems quite probable that the body of research previously identified would suggest that the most pathogenic CSA trauma responses are represented by the co-morbid presentations of dissociation and psychological distress and that family behaviors related to offending behavior incarcerated females; together suggest that incarcerated females may include some of the most pathological CSA presentations. 


\section{CHAPTER THREE}

\section{METHOD}

\section{Participants}

Incarcerated females between the ages of 18 years old and 65 years old $(M=33.18$, $\mathrm{SD}=8.65$ ) volunteered for research participation through recruitment notices posted in their dorms. The majority of the 157 participants, of varied ethnic origin, serve 1-15 year sentences for non-violent crimes at a southwest high-minimum security prison. This type of prison traditionally houses non-violent offenders but also individuals that represent a flight risk. The state prison is fence-enclosed and only 5 percent of the women work outside the prison on public work crews. One-sixth of the total prison population participates in a regimented treatment program whose members are housed separate from the general population and only interact with other prisoners in psycho-educational groups such as anger management and other cognitive-behavioral groups and in research projects such as the current investigation.

Demographic Characteristics of the Sample

Cultural Background: Of the 157 participants

- $52.9 \%$ identified themselves as Caucasian.

- $21.7 \%$ identified themselves as African American.

- $19.1 \%$ identified themselves as Native American.

- $5.1 \%$ identified themselves as Hispanic.

- $.6 \%$ identified themselves as Asian.

- $.6 \%$ identified themselves as "other." 


\section{Incarceration History:}

The mean number of separate incarcerations per individual of these 157 participants was 1.6 times $(\mathrm{SD}=.93)$ :

- $61.8 \%$ were serving for their first sentence.

- $21.7 \%$ were serving a second sentence.

- $12.1 \%$ were serving a third sentence.

- $3.2 \%$ were serving a fourth sentence.

- $\quad .6 \%$ were serving a fifth sentence.

- $.6 \%$ were serving a sixth sentence.

\section{Identified Childhood Caregiver}

Analysis of 157 participants' family structure in relation to primary caregiver(s) found:

- $33.8 \%$ were raised by both their mother and father.

- $43.8 \%$ were raised by their natural mother.

- $6.4 \%$ were raised by their mother and stepfather.

- $3.2 \%$ were raised by their maternal grandparents.

- $1.3 \%$ were raised by their paternal grandparents.

- $1.3 \%$ were raised by foster home parent or foster parents.

- $.6 \%$ were raised by their natural fathers.

- . $.6 \%$ were raised by "others."

Instrumentation

Background Information Sheet: The Background Information Sheet was the primary self-report measure (Zingman, 2003; See Appendix A) used to gather historical data related to the participant's primary caregiver(s), cultural background, incarceration 
history of self and relatives, abuse history, disclosure experiences and history of counseling after the abuse. Nine out of the fifteen questions related to history of abuse. These questions included the type of abuse, ages periods when abuse occurred (utilized by previous research--birth to seven years old; eight years old to twelve years old; and thirteen years old to eighteen years old), frequency of occurrence, relationship to the abuser, disclosure experiences (positive, negative, or not applicable and to whom) and history of counseling (yes versus. no; helpful, harmful, or not applicable). Information was also gathered on the occurrence of major life events such as deaths, community disasters, disruptive moves, different forms of accidents, etc).

This primary purpose of this measure was to separate participants with childhood sexual abuse histories from those with physical, emotional or no abuse. Directions on filling out this specific questionnaire were provided during both the general research introduction and after the four measures were distributed to participants. For this information sheet, participants were advised that questions would require an (X), a number value, or short description response. The questions related to history of abuse were preceded by definitions for each type of abuse.

Family Environment Scale: $3^{N D}$ Edition (FES-2) (Moos and Moos, 2002): The Family Environment Scale, a 90-item paper and pen instrument, was used in this research as a retrospective identification of the participant's family relationship dynamics from birth to 18 years old. Although the FES is designed to measure current social and environmental beliefs and attitudes, based on a three dimensional conceptualization of family interactions, it can be used as a retrospective measure. The dimensions addressed are: individual perceptions of current family (individual perception of one's family and their 
relationship to family members), individual preferences about an ideal family (actual versus ideal) or individual expectations about family settings (projected beliefs of what should occur in the future). The Real Form (Form R)-- Relationship Dimension, utilized by this investigation, addresses individual perceptions about the current or past family. The Form R has 90 questions of which 27 are related to the scales of cohesiveness, expressiveness, and conflict (nine questions related to each scale). Adding the number of keyed scores together, (scored for either its true or false value in relation to that family dynamic), together tabulates raw scores. Each subscale raw score is then identified with the score of same value score in the appropriate subscale's score column. Appropriate standardized scores are found parallel to the raw score. Normal ranges for each family relationship dynamics differ in their range across raw scores.

Reliability and validity for the FES have been established through extensive testing (Moos \& Moos, 1986) Internal consistency reliability estimates for the Form R range from .61 to .78. Test-retest reliability's for 2,3 , and 12-month intervals range from .52 to .91 . Very clear statements about family situations that relate back to the subscales support the face and content validity.

For use as a retrospective measure, directions were attached to the front of the participants' FES form reminding them to answer questions in relation to the family group or primary caretakers during the first 18 years of life and not in relation to the current family. The FES variable scores of Conflict, Expressiveness, and Cohesiveness can be separated into low, medium, and high levels. Such score ranges can be found in Moos and Moos (2002) Administration Manual's standardization tables for expected population means for distressed and non-distressed individuals. Mean scores for each of 
the three scales for the non-distressed population (defined by Moos and Moos, 2002) are utilized as the medium level score for each of the scales. The low and high division of scores were based on means for distressed individuals with cohesiveness and expressiveness being slightly lower than 1.5 standard deviations below the non-distressed respective means and conflict being slightly higher than 1.5 standard deviation above the non distressed conflict mean. In general, low and high scores of each score group are 1.5 standard deviations below the non-distressed mean of that scale.

Dissociative Experience Scale (DES) (Carlson \& Putnam, 1986; 1993): The DES is a 28 item self-report instrument designed to measure dissociation as levels of diminished integration of thoughts, experiences of stream of consciousness and lack of access to short and long term memory. Each question inquires how often the diminished experience occurs in the participant's daily life. Responses have value choices ranging from zero to 100 percent (in 10\% increments) of the time experienced. The authors of the DES do not view dissociation as a problem in itself, thus the scale was designed along a continuum from normal experiences to pathological dissociative experiences (without separation into separate personalities) of most trauma victims to the pathological dissociative experience of personality separation of a Dissociative Identity Disorder (DID) diagnosis. For scoring purposes, each item score (from 0 to 100) is added together and divided by 28 . The scoring manual notes that research shows that scores of 20 or above are indicative of recommending further diagnostic interviewing for possible dissociative tendencies or the diagnosis of a dissociative disorder. Scores of 30 or higher may represent a dissociatively distressed individual with the possibility of a DID diagnosis (Ross, 1992). 
The DES was designed using data from interviews with subjects diagnosed with dissociative disorders from the DSM III Criteria (1987) and from interviews with experts who work in the Dissociation field. It has been shown to have good split-half reliability (Ross, 1992) with coefficients from eight different groups ranging from .71 to .96 . Stability found in a four to eight week test-retest produced a reliability coefficient of .84 . The DES has been found to have good test-retest reliability, internal consistency, construct validity and criterion related validity in clinical and non-clinical samples (Carlson \& Putnam, 1986). The ability of group median scores to differentiate DID from other diagnostic groups have been replicated, showing strength in predicting DID or other forms of dissociative disorders. Many of the DES dimensions have been shown to be highly reliable and internally consistent when compared to other measures that define similar dimensions from the MMPI -2 and the SCL-90-R DISS Scale (Dubester and Braun, 1995; Bernstein and Putnam, 1986).

Symptom Checklist-90-Revised (SCL-90-R)(Derogatis, 1994): The SCL-90-R is a brief multi-dimensional self-report measure designed to screen for a broad range of psychological problems and symptoms of pathology. It can be used for primary evaluation of symptom presentation, progress analysis during therapy and for research. The SCL-90-R is a 90-item questionnaire with a 5-point scale. Questions are grouped into 9 primary symptom dimensions whose grouped scores are tabulated into 3 global scales. The individual question's scores are interpreted as current point-in-time measures of psychological symptom status. The nine, empirically derived, symptom dimensions are; Somatization (SOM), Obsessive-Compulsive (OC), Interpersonal Sensitivity (IS), Depression (DEP), Anxiety (ANX), Hostility (HOS), Phobic Anxiety (PHOB), Paranoid 
Ideation (PAR) and Psychoticism (PSY). The three global Indexes are the Positive Symptom Total (PST), the Global Severity Index (GSI), and the Positive Symptom Distress Index (PSDI).

The SCL-90-R's was designed for 6th grade reading level abilities and takes about 12 to 15 minutes to complete. It has been normed against both adults and adolescents in the general population, both inpatient and out patient groups and a wide variety of cultural groups. For each separate question of the nine primary symptom dimensions respondents indicate the level of intensity experienced for a specific emotional dynamic on a fivepoint scale ranging from 0 -- "not at all" to 5 -- "extremely." Adding the nine primary symptom dimensions' intensity scores tabulates GSI scores. A high GSI score (above a Tscore of 63 ) is derived from more than $50 \%$ of the questions being endorsed as occurring as "extremely" (almost all of the time) or "quite a bit" (more often than not). This high level of endorsement, more often than not, means the individual identified almost daily experience of the psychological symptom. A GSI T-score of 63 or higher has been defined by Derogatis and Lazarus (1994) as indicative of possible pathology and such a score was used for analysis of impact of CSA and family relationship dynamics.

Derogatis and Lazarus (1994) report good internal consistency (.75 to .85) and good test retest reliability $(.75$ to .90$)$. The SCL-90-R has been translated and normed into 20 languages and is used extensively in cross-cultural research (Kim, Kim \& Won, 1983). The SCL-90-R has also been successfully used to test the convergent validity of the DES (Gold et al., 1999). Although though the SCL-90-R appears to be trauma sensitive, few trauma studies have utilized this measure.

Although the GSI is designed to assess the summary of the individual distress rather 
than individual symptoms, individual scales can be analyzed in relation to their contribution to the GSI score. For purpose of this research, individual scale scores were not analyzed. The GSI scores, along with DES scores were used as dependent variables measuring the impact of CSA and family relationship dynamics of cohesiveness, expressiveness, and conflict.

\section{Procedure}

Postings advertised the research as investigating how childhood events and family relationships affect adult personality and coping styles. Although the research's focus was related to sexual abuse and family, it was decided that a slight deception should be used in relation to the sexual abuse focus. Due to sensitivity related to disclosure of sexual abuse, CSA was not listed as the primary focus of the research but rather a topic of some of the questions. The postings clearly identified that no monetary award or credit would be given for participation. (See Appendix F). Even slight deception was reticently used due to the historical nature of deception used within prison population interactions (Farrington, 1995).

The four pencil and paper instruments, randomly ordered, were handed out face down to the participants. Two copies of the Informed Consent Form (See Appendix B) form were disbursed with the test packets. During the instruction period (See Instruction Script - Appendix F) consent forms were reviewed. The experimenter reminded participants that signed consent forms would be kept separate from the measures. Participants were further reminded that if individuals did not want their consent forms to be part of a random sample sent to the Department of Corrections' administration office, they should check the box on the form. They were instructed to not write names on test forms. 
Because prisoners expressed concern about staff gaining access to personal information, reassurance was given several times during each pre-test instruction period and during administration, that confidentiality would be protected. The experimenter explained that only participants themselves could choose to break confidentiality. In the event that the participant became extremely distressed during or after the administration of tests, she could choose to break confidentiality by requesting a referral to Mental Health Services and signing a form included with the testing packet.

During the introduction, the process of answering the four types of questionnaires was reviewed, with recognition that each measure had instructions printed on the first page. Participants were also reminded to answer the FES form based on their life from birth to 18 years old and not in relation to the current family.

After the instruction period, it took individuals, in groups averaging from 20 to 30 participants, anywhere from 45 minutes to an hour to complete the four questionnaires. Six research sessions were conducted to achieve the $\mathrm{N}$ of 157 . During testing, individuals were allowed to raise hands for help on instructions. An assistant, trained by the researcher, handed out packets and help observe participants during the administration in order to identify any individual distress over the research subject and to answer procedure questions. The participants brought their packets forward when they finished, had consent forms signed by the administrator and received their own copy. They also received a disclosure/ debriefing sheet (See Appendix D) that explained the complete purpose of the research, rationale for the use of slight deception, follow-up procedures concerning complaints, obtaining results of the experiment and referrals for dealing with any issues raised by the research procedure. 


\section{Design of the Study}

The design of the study was a $2 \times 3$ multivariate analysis of variance (MANOVA). The

two independent variables were history of childhood sexual abuse (yes or no) and level of family relationship dynamic (low, medium and high levels for scores of conflict, expressiveness and cohesiveness). The dependent variables were scores of the Dissociative Experience Scale and scores of the Global Severity Index of the SCL-90-R. Three separate MANOVAs were run; one for each family relationship variable.

For the independent FES variable scores, divisions of high, medium and low score groups were based on research findings comparing symptomatic populations to normal populations, more specifically, on Moos \& Moos (2002) administration manual's standardization tables for expected population means for distressed and non-distressed individuals. As noted in the Instrumentation section, low, medium, and high score ranges for each FES group were based on medium - normal score means of conflict, expressiveness and cohesiveness for non-distressed individuals and 1.5 standard deviations from the normal score for lower than normal family dynamics or higher than usual family dynamic. As noted earlier Conflict scores were separated by; low $=33-45$, medium $=46-54$, high $=55-80 ;$ Expressiveness scores were separated by low $=16-49$, medium $=50-56$, high $=57-71 ;$ Cohesiveness scores were separated by: low $=33-45$; medium $=46-54 ;$ high $=55-80$.

For purpose of interpretation, the DES groupings of score ranges were noted as being non-clinical levels of dissociation, with slight loss of awareness of time and place, for scores from 0-20. Medium levels of dissociation from 20 to 30 were assessed as more disruptive to everyday consciousness and have been associated with symptom responses 
to traumatic experiences. High levels of dissociation were assessed for scores above $\mathbf{3 0}$ that were representative of the most pathological dissociation and could possibility some form of Dissociative Identity Disorder (Carlson \& Putnam, 1993; Gold et al., 1990; Greaves, 1993; Kluft, 1990; Ursano \& Fullerton, 1990). Analysis of this research's findings, utilized any score above 20 as a significant symptomatic presentations of dissociation (medium to high).

Variables

Independent Variables

The independent variables of this research were two levels of sexual abuse (a history of sexual abuse or the absence thereof) and three levels of family functioning (high, medium and low) for each of the three family relationship dynamics: cohesiveness, expressiveness, and conflict (Moos \& Moos, 2002).

\section{Dependent Variables}

The dependent variables of this research were dissociation scores as measured on the Dissociative Experience Scale (Carlson \& Putnam, 1993) and scores from the Global Severity Index (GSI) of the SCL-90-R (Derogatis, 1994). GSI scores were utilized as indicating overall psychological distress, rather than number of symptoms present. 


\section{CHAPTER FOUR}

\section{RESULTS}

\section{Introduction}

The purpose of this study was to examine relationships between the independent variables of a childhood sexual abuse history and levels of perceived childhood family expressiveness, cohesiveness and conflict, with the dependent variables levels of dissociation (DES Scores) and levels of psychological distress (GSI scores). Chapter 4 presents the statistical analysis of results. The correlation between GSI and DES scores was significant $(\mathrm{r}=.61 ; \mathrm{p} .=009)$, thus a multivariate analysis of variance was conducted. General Analysis of Results

Occurrence of Sexual Abuse: Of the total participant pool, more than 59\% reported a CSA history with the following breakdown for time-period of occurrence:

- 26 women identified sexual abuse occurring during all three age periods: 1) birth to seven years old, 2) eight years old through twelve years old 3) thirteen years old to eighteen years old.

- 17 women identified abuse during the first two age periods

- 13 women identified abuse during the first age period

- 12 women identified abuse during the second age period

- 10 women identified abuse during the last age period

- 6 women identified abuse during the second and last age periods

- 6 women identified abuse during the first and last age periods. Counseling After Abuse:

Of the 90 participants claiming a sexual abuse history, 89 participants (57\%) identified no counseling after the abuse. 


\section{Disclosure Experiences:}

For the 90 female prisoners identifying a sexual abuse history, 74 of these women identified disclosure experiences after the abuse. Of this group, 45 indicated these experiences as harmful, not helpful.

\section{Breakdown of DES and GSI Scores for Participants}

As expected, DES and GSI scores for the 157 participants generally represent a very symptomatic population. DES scores had a mean of 18.37 (standard deviation of 15.14). GSI scores had a mean of 63.76 (standard deviation of 12.26). For the $59 \%$ of participants who identified a CSA history, $70 \%$ had DES scores above 21 , with $14 \%$ obtaining scores above 30 . More than $55 \%$ of those with a CSA history had GSI scores above 63 , with $9.9 \%$ of these scores above 80 .

\section{Statistical Analysis}

Research Question 1: What is the relationship of the independent variables of a remembered childhood sexual abuse history and levels of perceived childhood family conflict (CON-FES scores) with the dependant variables of Dissociative magnitude (DES scores) and levels of psychological distress (GSI SCL-90-R scores)?

Null Form of Hypothesis I: (See Chapter 1) There is no relationship between a remembered childhood sexual abuse history and perceived childhood family conflict with magnitude of dissociation and levels of psychological distress.

It was hypothesized that there would be a relationship between the independent variables of a sexual abuse history and childhood family conflict with the dependant variables of dissociation and psychological distress.

In order to test Null Hypothesis I, a 2 X 3 (Sexual Abuse by level of Family Conflict) multivariate analysis of variance of DES and GSI scores was conducted. A significant 
main effect was found for sexual abuse $(F=4.88 ; \mathrm{p}=.009 ; \mathrm{df}=2,150)$ and for conflict $(F=2.42 ; p=.048 ; d f=4,302)$. No significant interaction effect was found for sexual abuse by family conflict $(F=1.33 ; p=.259 ; \mathrm{df}=4,302)$. In that a significant main effect was found for sexual abuse and for family conflict, a follow-up analysis was conducted to assess which dependant variable scores contributed more to the main effect of each of the independent variables. Univariate (one-way) analysis showed that the main effect of sexual abuse was significant for both DES $(\mathrm{F}=7.43, \mathrm{p}=.007$; $\mathrm{df}=1,155)$ and GSI scores $(\mathrm{F}=5.28, \mathrm{p}=.023 ; \mathrm{df}=1,155)$. Therefore, the main effect of sexual abuse was equally distributed across both DES and GSI scores. Univariate (one-way) analysis showed that the main effect of conflict was significant for DES scores $(F=5.13, p=$ $.007 ; \mathrm{df}=2,154)$ but not for GSI scores $(\mathrm{F}=2.63, \mathrm{p}=.075 ; \mathrm{df}=2,154)$. Therefore, the main effect of family conflict was due primarily to the impact of DES scores rather than GSI scores. Post Hoc Scheffe tests showed that there was a significant difference between DES mean scores of low and high family conflict with the high conflict having the highest DES mean score $(\mathrm{Sig} .=.003$; Mean dif. $=9.82)$. No other comparisons were significant.

Means and standard deviations of DES and GSI scores across levels of family conflict are presented in Tables 1 and 2 respectively. A summary table of MANOVA results for sexual abuse by family conflict analysis is presented in Table 3 .

Research Question 2: What is the relationship of the independent variables of a remembered childhood sexual abuse history and levels of perceived childhood family expressiveness (FES-EX scores) with the dependant variables of Dissociative magnitude (DES scores) and levels of psychological distress (GSI SCL-90-R scores)?

Null Form of Hypothesis II: There is no relationship between a remembered childhood 
Table 1 Means and Standard Deviations of Dissociative Experience Scale

Scores For Sexual Abuse by Family Conflict Analysis

\begin{tabular}{|c|c|c|c|c|c|c|c|c|c|}
\hline Sexual & \multicolumn{2}{|c|}{ Low } & \multicolumn{2}{|c|}{ Medium } & \multicolumn{2}{|c|}{ High } & \multicolumn{3}{|c|}{ Row } \\
\hline $\begin{array}{l}\text { Abuse } \\
\text { History }\end{array}$ & Mean & SD & Mean & SD & Mean & $\mathrm{N}^{\mathrm{SD}}$ & Mean & $\mathrm{N}$ & SD \\
\hline Yes & 16.73 & 10.95 & 22.76 & 15.90 & 22.76 & $63^{15.60}$ & 21.16 & 90 & 15.00 \\
\hline No & 5.99 & 6.87 & 13.57 & 13.99 & 19.06 & $35^{15.84}$ & 14.63 & 67 & 14.60 \\
\hline $\begin{array}{r}\text { Column } \\
\text { Total }\end{array}$ & 11.36 & 10.38 & 18.69 & 15.35 & 20.82 & $98^{15.87}$ & 18.37 & 157 & 15.84 \\
\hline
\end{tabular}


Table 2 Means And Standard Deviations Of Global Severity Index Scores For Sexual Abuse By Family Conflict Analysis

\begin{tabular}{|c|c|c|c|c|c|c|c|c|c|c|}
\hline \multirow{2}{*}{$\begin{array}{l}\text { Sexual } \\
\text { Abuse } \\
\text { History }\end{array}$} & \multicolumn{2}{|c|}{ Low } & \multicolumn{3}{|c|}{ Medium } & \multicolumn{3}{|c|}{ High } & \multicolumn{2}{|c|}{ Row Total } \\
\hline & Mean & SD & Mean & $\mathrm{N}$ & SD & Mean & $\mathrm{N}$ & SD & Mean & $\mathrm{SD}$ \\
\hline Yes & 66.42 & 7.86 & 64.53 & 13 & 8.12 & 65.74 & 63 & 13.63 & $\begin{array}{r}65.57 \\
9\end{array}$ & 12.14 \\
\hline No & 54.62 & 10.40 & 59.68 & 16 & 10.16 & 64.88 & 35 & 12.30 & 61.15 & 2.01 \\
\hline $\begin{array}{l}\text { Column } \\
\text { Total }\end{array}$ & 60.52 & 7.86 & 62.11 & 29 & 8.12 & 65.31 & 98 & 13.11 & $\begin{array}{r}63.76 \\
15\end{array}$ & $7^{12.26}$ \\
\hline
\end{tabular}


Table 3 MANOVA Summary for Sexual Abuse by Family Conflict

\begin{tabular}{l|c|c|c|c|c} 
& Effect & $\mathrm{F}$ & $\begin{array}{c}\text { Hypothesis } \\
\mathrm{df}\end{array}$ & $\begin{array}{c}\text { Error } \\
\mathrm{df}\end{array}$ & Sig. \\
\hline $\begin{array}{l}\text { Sexual Abuse } \\
\text { (Pillai's Trace) }\end{array}$ & .061 & 4.88 & 2.00 & 150.00 & .009 \\
$\begin{array}{l}\text { Conflict } \\
\text { (Pillai's Trace) }\end{array}$ & .062 & 2.42 & 4.00 & 302.00 & .048 \\
$\begin{array}{c}\text { Sexual Abuse } \\
\text { by Conflict } \\
\text { (Pillai's Trace) }\end{array}$ & .035 & 1.33 & 4.00 & 302.00 & .259 \\
\hline
\end{tabular}


sexual abuse history and perceived childhood family expressiveness with magnitude of dissociation and levels of psychological distress.

It was hypothesized that there would be a relationship of the independent variables of sexual abuse and family expressiveness with the dependant variables of DES scores and GSI scores.

In order to test Null hypothesis II a 2x3 (Sexual Abuse by level of Family Expressiveness) multivariate analysis of variances (MANOVA) of DES and GSI scores was conducted. A significant main effect was found for sexual abuse $(F=3.55$; $\mathrm{p}=.031 ; \mathrm{df}=2,150)$ and for family expressiveness $(\mathrm{F}=2.66 ; \mathrm{p}=.032 ; \mathrm{df}=4,302)$. No significant interaction was found for sexual abuse and family expressiveness $(\mathrm{F}=2.36, \mathrm{p}=.053 ; \mathrm{df}=4,302)$.

In that a significant main effect was found for both independent variables, a follow-up analysis assessed which dependant variables contributed more to the main effect of the independent variables. Utilizing a univariate (one-way) analysis, it was found that for sexual abuse, the main effect was due to both DES scores DES $(F=7.43, p=.007 ; \mathrm{df}=$ $1,155)$ and GSI scores $(\mathrm{F}=5.28, \mathrm{p}=.023 ; \mathrm{df}=1,155)$. Therefore, the main effect of sexual abuse was equally distributed across both DES and GSI scores. The main effect for family expressiveness was significant for DES scores $(F=3.21 ; p=.043 ; \mathrm{df}=2,154)$ and GSI scores $(\mathrm{F}=3.26, \mathrm{p}=.041 ; \mathrm{df}=2,154)$. Post Hoc Scheffe tests showed that there was a significant difference between DES mean scores of low and high family expressiveness with high family expressiveness having the highest score $(\mathrm{Sig} .=.046$; Mean dif. $=7.49$ ). No other comparisons were significant.

Means and standard deviations of DES and GSI scores across levels of family expressiveness are presented in Tables 4 and 5, respectively. A summary table of 
Table 4 Means And Standard Deviations Of Dissociative Experience Scale Scores For Sexual Abuse By Family Expressiveness Analysis

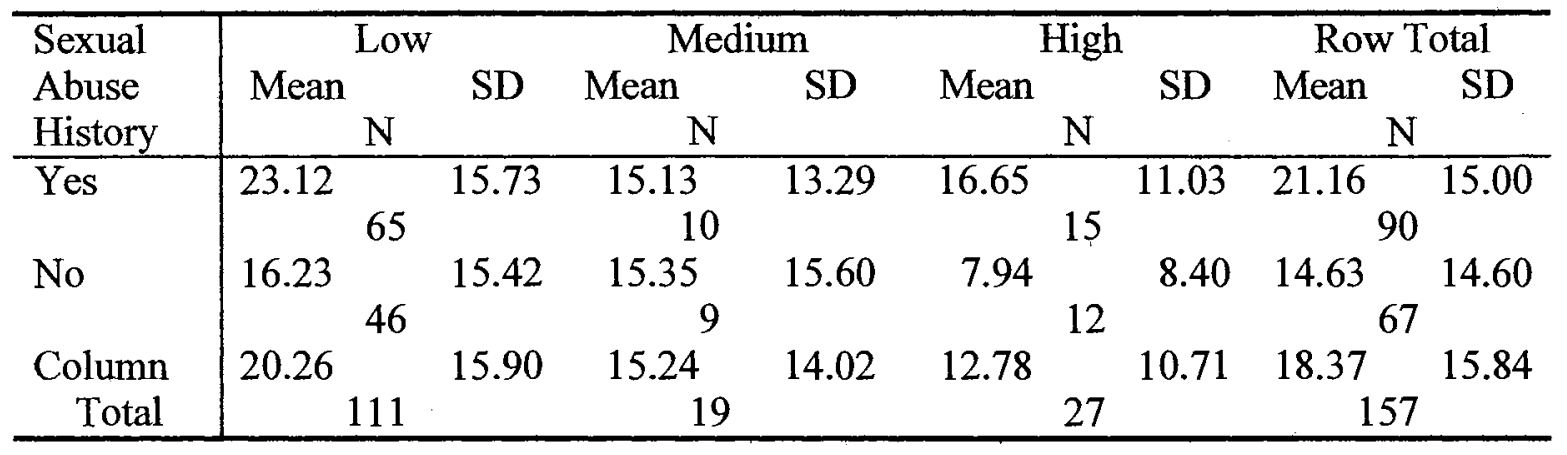


Table 5 Means And Standard Deviations Of Global Severity Index Scores For Sexual Abuse By Family Expressiveness Analysis

\begin{tabular}{|c|c|c|c|c|c|c|c|c|}
\hline Sexual & Low & & Mediun & & High & & Row & tal \\
\hline $\begin{array}{l}\text { Abuse } \\
\text { History }\end{array}$ & \multicolumn{2}{|l|}{ 19 } & \multicolumn{2}{|l|}{ thena } & \multicolumn{2}{|l|}{ 17. } & Mean & $\cdot$ \\
\hline Yes & 65.81 & 12.83 & 65.30 & 9.10 & 65.31 & 11.51 & 65.57 & 12.14 \\
\hline No & 63.73 & 8.16 & 59.68 & 10.16 & 64.88 & 12.30 & 61.15 & 12.01 \\
\hline $\begin{array}{c}\text { Column } \\
\text { Total }\end{array}$ & 64.95 & 8.16 & 64.47 & 8.55 & 58.37 & 12.75 & $\begin{array}{r}63.76 \\
15\end{array}$ & 12.26 \\
\hline
\end{tabular}


MANOVA results for sexual abuse by family expressiveness analysis is presented in Table 6.

Research Question 3: What is the relationship of the independent variables of a remembered childhood sexual abuse history and levels of perceived childhood family cohesiveness (FES-COH scores) with the dependant variables of Dissociative magnitude (DES scores) and levels of psychological distress (GSI SCL-90-R scores)?

Null Form of Hypothesis III: There is no relationship between a remembered childhood sexual abuse history and perceived childhood family cohesiveness with magnitude of dissociation and levels of psychological distress.

It was hypothesized that there would be a relationship of the independent variables of sexual abuse and family cohesiveness with the dependant variables of DES scores and GSI scores. In order to test Null hypothesis III a 2x3 (Sexual Abuse by level of Family Cohesiveness) multivariate analysis (MANOVA) of variances of DES scores and GSI scores was conducted. A significant main effect was found for sexual abuse $(F=3.30$; $\mathrm{p}=.039 ; \mathrm{df}=2,150$ ) but no significant main effect was found for family cohesiveness $(\mathrm{F}=1.55 ; \mathrm{p}=.189 ; \mathrm{df}=4,302)$. No interaction was found for CSA by family cohesiveness $(\mathrm{F}=.237, \mathrm{p}=.918 ; \mathrm{df}=4,302)$.

In that there was a significant main effect for sexual abuse, follow-up analysis assessed which dependent variable contributed more to the main effect of the independent variables. Univariate (one-way) analysis found that for sexual abuse, the main effect was significant for DES scores $(\mathrm{F}=7.43, \mathrm{p}=.007 ; \mathrm{df}=1,155)$ and $\mathrm{GSI}$ scores $(\mathrm{F}=5.28, \mathrm{p}=$ $.023 ; \mathrm{df}=1,155)$. Although no significant impact was found for family cohesiveness the Means and standard deviations of DES and GSI scores across levels of family cohesiveness are presented in Tables 7 and 8 , respectively. A summary table of the 
MANOVA results for sexual abuse by Family Cohesiveness analysis is presented in

Table 9. 


\begin{tabular}{c|ccccc} 
Effect & Value & $\mathrm{F}$ & $\begin{array}{c}\text { Hypothesis } \\
\mathrm{df}\end{array}$ & $\begin{array}{c}\text { Error } \\
\mathrm{df}\end{array}$ & Sig. \\
\hline $\begin{array}{c}\text { Sexual Abuse } \\
\text { (Pillai's Trace) }\end{array}$ & .045 & 3.55 & 2.00 & 150.00 & .031 \\
$\begin{array}{c}\text { Expressiveness } \\
\text { (Pillai's Trace) }\end{array}$ & .068 & 2.665 & 4.00 & 302.00 & .033 \\
$\begin{array}{c}\text { Sexual Abuse by } \\
\text { Expressiveness } \\
\text { (Pillai's Trace) }\end{array}$ & .061 & .236 & 4.00 & 302.00 & .053 \\
& & & & & \\
\end{tabular}


Table 7 Means And Standard Deviations Of Dissociative Experience Scale Scores For Sexual Abuse By Family Cohesiveness Analysis

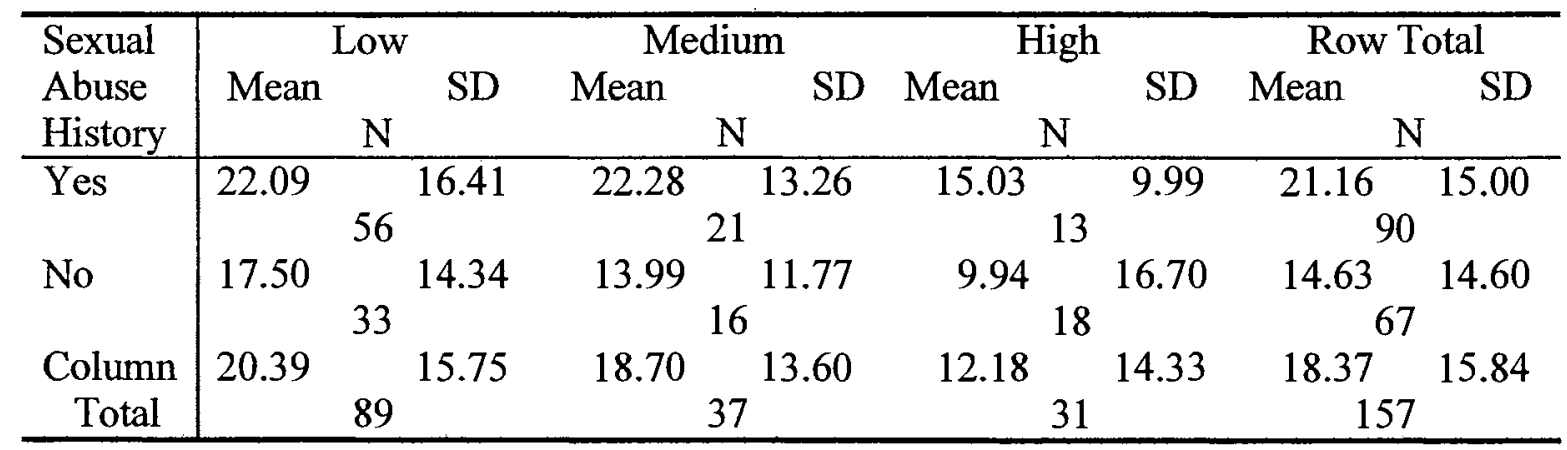


Table 8 Means And Standard Deviations Of Global Severity Index Scores For Sexual Abuse By Family Cohesiveness Analysis

\begin{tabular}{|c|c|c|c|c|c|c|c|}
\hline Sexual & \multicolumn{2}{|c|}{ Low } & \multicolumn{2}{|c|}{ Medium } & \multicolumn{2}{|c|}{ High } & Row Total \\
\hline $\begin{array}{l}\text { Abuse } \\
\text { History } \\
\end{array}$ & Mean & SD & Mean & SD & Mean & $\begin{array}{ll} & \mathrm{SD} \\
\mathrm{N} & \\
\end{array}$ & ${ }_{\mathrm{N}}^{\text {Mean }}{ }^{\text {SD }}$ \\
\hline Yes & 65.75 & 5 & 67.28 & 10.10 & 62.62 & $13^{12.20}$ & ${ }_{90}^{65.57}{ }^{12.14}$ \\
\hline No & 63.36 & 11.07 & 61.62 & 13.08 & 56.63 & $18^{12.20}$ & $61.15{ }_{67}^{12.01}$ \\
\hline $\begin{array}{c}\text { Column } \\
\text { Total }\end{array}$ & 64.47 & 12.33 & 64.83 & 11.66 & 59.29 & $31^{12.09}$ & $\begin{array}{c}63.76 \quad 12.26 \\
157\end{array}$ \\
\hline
\end{tabular}


Table 9 MANOVA Summary For Sexual Abuse By Family Cohesiveness

\begin{tabular}{c|ccccc} 
Effect & Value & $\mathrm{F}$ & $\begin{array}{c}\text { Hypothesis } \\
\mathrm{df}\end{array}$ & $\begin{array}{c}\text { Error } \\
\mathrm{df}\end{array}$ & Sig. \\
\hline $\begin{array}{c}\text { Sexual Abuse } \\
\text { (Pillai's Trace) }\end{array}$ & .042 & 3.303 & 2.00 & 150.00 & .39 \\
$\begin{array}{c}\text { Cohesiveness } \\
\text { (Pillai's Trace) }\end{array}$ & .040 & 1.545 & 4.00 & 302.00 & .189 \\
$\begin{array}{c}\text { Sexual Abuse by } \\
\text { Cohesiveness } \\
\text { (Pillai's Trace) }\end{array}$ & .006 & .237 & 4.00 & 302.00 & .917 \\
\end{tabular}




\section{CHAPTER FIVE}

\section{DISCUSSION}

Introduction

Conception and design of this research was based on both the premise that underresearched populations may include highly symptomatic childhood sexual abuse survivors and that incarcerated females may represent such a group. It was also suggested that female prisoners would show some of the most debilitating and complex sexual abuse-based trauma response profiles, primarily consisting of high levels of dissociation and psychological distress. Furthermore, it was suggested that levels of symptom intensity would, in some way, be related to the combined experience of childhood sexual abuse and the female prisoner's perceived childhood history of family conflict, family expressiveness, and family cohesiveness. Interpretation of results is found in the Major Findings and the Implications sections of this chapter. Chapter 5 presents the complete discussion of research findings in the following manner: Limitations of the Study, Major Findings, Implications, Suggestions for Future Research and Summary and Conclusions. Limitations of the Study

Limitations for this study were both standard limitations found in most quasiexperimental research as well as being population-specific for female prisoners. In general, studies based on self-selected population samples can result in biased findings if individual motivation for participation skews answers in relation to the motivation (Derogatis and Lazarus, 1994). For example, daily prisoner functioning is often based on rewards and punishment (Sweezy, 1998). Some participants in this study may have been motivated by perceived positive or negative benefits in their relationships with staff, rather than on sharing self-knowledge. Prisoner attitudes that staff would look positively 
on participation, or negative for lack of, may have prompted some participants to "just get the process done" without attention to detail. Such motivation was possibly reinforced by confidentiality procedures (as in all research projects) that identified participant answer sheets by number and not by name. Research participant instructions, read and explained at the beginning of each administration, noted this level of confidentiality. In that level of participation could, therefore, not be established, this may have made it easier for an individual to provide only the appearance of participation. Even so, assessment of scores and individual answers did not appear to show random answering patterns. DES and GSI scores, as predicted, also appeared consistently higher for those with sexual abuse histories (See Table 1 and Table 2; Table 4 and Table 5; Table 7 and Table 8).

Another built-in limitation originates in the assumption that individuals will answer self-report questionnaires in a truthful manner (Derogatis \& Lazarus, 1994). As noted in previous sections, many environmental factors within the prison system influence a prisoner's willingness, or ability to identify current emotional states. Analysis of respondents' answer sheets with extremely low or high scores for both the DES and GSI scale appeared to show that some inmates may have attempted to "look good" and some may have attempted to "look bad." The "faking bad" appeared, for some, to be a "cry for help." Almost all of the individuals with extremely high DES and GSI scores also signed a release form relinquishing anonymity so they could receive a referral to counseling based on analysis of their answers. In that female prisons are typically under-staffed and there are often long waits for mental health services, it is suggested that these participants may have assumed that increased symptom endorsement increased chances for a referral. On the other hand, individuals who appeared to "fake good" may have been reacting to 
the invasive nature of the prison system and did not trust the confidentiality enough to even endorse low levels of symptom presence. Thus, the "truthful" assumption may be suspect.

The sensitive nature of family history and abuse questions had the potential to limit some participants' from either completing all questionnaires and/or openly answering all questions. It may have been too threatening for some individuals, especially those without any history of mental health interventions, to address memories and emotions related to childhood families and sexual abuse. Such an influence was hopefully limited by reminding participants at the introduction, and twice during administration, that, if uncomfortable with the nature of the questions, they could terminate their participation and leave without reprimand. Only two participants left during administration. It is hoped that the $\mathrm{N}$ of 157 was a large enough to help neutralize the (negative) impact of limitations listed above.

This study, although including many members' of under-researched cultural populations, is limited in generalizing results to support theories about specific culturerelated responses to childhood sexual abuse and dysfunctional childhood family dynamics. Although culture representation was diverse, there were not enough representatives of African Americans, Hispanic, Native American, or Asian cultures to deduce any significant population-specific data. Thus, replication studies with specific populations of similar ethnic mix must build upon this study's findings.

In relation to the specific study of dissociation and sexual abuse, some individuals, experiencing high levels of dissociation, may not be able to recall certain feelings or memories related to the abuse experience or to their childhood family's interactions (Everstine \& Everstine, 1993; Fredrickson, 1992; Kluft, 1990). This may have suppressed 
some DES or GSI scores, may have lowered the number of identified sexual abuse histories, and thus limited the identification of some associations between childhood family relationship dynamics and sexual abuse on levels of dissociation and psychological distress.

In general, the limitations of this study lead to some question about the validity of findings. Even so, the consistently higher levels of dissociation and psychological distress for those endorsing a sexual abuse history and dysfunctional childhood family dynamics, when compared to those without sexual abuse histories, suggests the limitations had minimal effect on research findings.

Major Findings

1. A significant main effect was found for CSA across both the dependent variables of dissociation and psychological distress. Specifically, it was consistently found that, for female prisoners, childhood sexual abuse survivors had higher levels of dissociation and psychological distress than individuals who did not report a history of childhood sexual abuse. These findings also suggest, for the female prisoner sexual abuse survivor population, there is a co-morbid existence of clinical levels of dissociation and psychological distress.

2. A significant main effect was found for levels of childhood family conflict on overall levels of dissociation and psychological distress with the primary effect due to conflict's influence on levels of dissociation. Specifically, multivariate and univariate (one-way) analysis showed that, for female prisoners, childhood family conflict had significant impact on levels of dissociation but not psychological distress. Furthermore, this significance was only found in the difference between high and low levels of family conflict. 
3. A significant main effect was found for levels of childhood family expressiveness on overall levels of dissociation and psychological distress with the main effect due to expressiveness' influence on both levels of dissociation and psychological distress. Specifically, multivariate and univariate (one-way) analysis showed that, for female prisoners, childhood family expressiveness had a significant impact on levels of dissociation and psychological distress. Further post hoc analysis showed that the primary significant difference was found for DES scores for those who endorsed low and high expressiveness with the low expressiveness group having the highest DES scores.

4. No main effect was found for family cohesiveness on overall levels of dissociation and psychological distress. Thus, for female prisoners, levels of childhood family cohesiveness do not have any influence on levels of dissociation or psychological distress.

5. No interaction effects were found for sexual abuse and levels of childhood family conflict, expressiveness, or cohesiveness on levels of dissociation and psychological distress. Thus, when looking at female prisoners' dissociation and psychological distress, similar symptom patterns were found across all levels of each of the three childhood family dynamics, regardless of the individual's childhood sexual abuse history.

\section{Implications}

In general, this study yielded evidence that incarcerated females are a highly symptomatic population with many unresolved mental health issues, that female prisoners have high rates of sexual abuse histories, that clinically significant levels of dissociation and psychological distress are prevalent in many of the female prisoners' symptom presentations. Furthermore, for those prisoners with a sexual abuse history, the 
associated symptom profiles were significantly related to the impact of a sexual abuse history and/or the endorsed childhood family relationship dynamics of low expressiveness and high conflict (See Tables 1-9).

The identification of female prisoners as a highly symptomatic population was established by findings of co-morbid presentations of high levels of dissociation and psychological distress. An overall population mean $(n=157)$ of 18.37 for DES scores and 63.76 for GSI scores, in conjunction with $57 \%$ of participants reporting a sexual abuse history with a DES mean score of 21.7 and a GSI mean score of 65.7 , identifies the majority of this population experiencing clinical levels of these symptoms. This interpretation is further supported by the statistically tight clustering of participants' DES and GSI scores, with the majority of scores in the high range. Clinical interpretation of standardized scores shows that DES scores of 21 or higher are indicative of traumarelated dissociative symptoms and suggest further evaluation of dissociative diagnosis, while scores of 30 or above may be indicative of a Dissociative Identity Disorder diagnosis (Carlston \& Putnam, 1993). GSI scores greater than 63 have predictive capability for an individual to be at risk for some form of psychiatric disorder (Derogatis, 1994). In that the majority of these female prisoners experience dissociation and psychological distress at clinically significant levels rather than across a wide range of levels, it is suggested that such a large percentage of co-morbid clinically significant presentations might represent some sort of reciprocal or parallel relationship between the symptom clusters. Otherwise, it would be expected that levels of dissociation and psychological distress would vary across a wide range of scores.

The identification of the co-morbid existence of dissociation and psychological distress challenges logic that suggests an individual experiencing high levels of 
dissociation would not be subjected to, or aware of, high levels of psychological distress. It appears that for incarcerated women, even though dissociation provides a type of barrier from unresolved emotional issues (as indicated by high levels of dissociation), during non-dissociative periods these individuals may be acutely aware of and affected by (as indicated by high levels of psychological distress) their emotional issues and by environmental triggers (Bloch, 1991; Collins \& Ffrench, 1998; Lynn \& Rhue, 1994). These co-morbid symptom profiles may also identify the prisoner's dichotomous need to be both hypervigilant towards, and distance one's self from, environmental triggers (Sweezy, 1998). More specifically, female prisoners' symptom responses could be affected by the power-oriented, at times abusive, supervision of mostly male guards, and the punitive structure of prison life. Routines and sometimes-paternalistic attitudes, combined with limited privacy or respect of personal boundaries (Gorosuch, 1998; Henderson et al., 1998) may mimic similar environmental abuse dynamics experienced by victims of childhood sexual abuse. Thus, when the female prisoners are not protected by dissociation's distancing ability, their environment-related anxiety escalates.

It is also interesting to note that Haberstadt et al. (1995) found that low expressiveness was a predictor of a high rate of anger and affect intensity. Anger regulation has been noted as a major issue for offenders, for prison staff and for maintaining security within prisons (Gorsuch, 1998). Higher levels of psychological distress for these female prisoners may be further compounded by, and may partially represent this unregulated anger and affect intensity.

Lenore Terr (1991) suggests that long-term abuse, if not processed emotionally creates an inability to process feelings, a cognitive disengagement from these feelings (through dissociation), inaccessible rage and sadness and guilt and shame associated with the 
abuse. Given the lack of clinical intervention for this population (57\%) and taking into consideration this population's high levels of dissociation and psychological distress, a preliminary assumption, (in support of Terr's findings) can be made that, for female prisoners, the lack of intervention after childhood sexual abuse may partially predict high levels of dissociation and psychological distress.

The female prison population, with such a high population percentage of childhood sexual abuse survivors, mirrors findings for clinical sexual abuse populations more so than for college sexual abuse populations (Coyne \& Schwenk, 1997). Even so, much of the resemblance to clinical populations stops at this comparison. As Sweezy (1998) suggested, female prisoners represent a group of highly symptomatic sexual abuse survivors who are not consumers of mental health services. Gold (2000) notes that there may be large groups of sexual abuse survivors who are also not part of normally researched clinical or college populations. Thus research statistics probably underestimate the number of these highly symptomatic sexual abuse survivors due to their membership in under-researched and under-treated populations. This is true about female prisoners but not usually true about clinical populations.

Even with the demographic differences between female prisoners and clinical populations, it was not surprising that female prisoners' levels of psychological distress mirror psychiatric populations. Past research, in many ways, predicts such an outcome. As previously noted (Derogatus \& Lazarus, 1994), high levels of psychological distress indicate the existence of numerous anxiety provoking experiences rooted in a mix of clinically significant psychological symptoms; many of which make up various mood disturbances. Coyne and Schwenk (1997) found that psychological distress is a strong indicator of the presence of mood disturbance (a diagnosis for many counseling and 
hospitalized clients) and impairment of functioning for psychiatric populations yet not for college populations. In conjunction with the above findings, Swett, Surry and Cohen (1990) showed that psychiatric patients with sexual abuse histories have significantly higher levels of psychological distress (clinical GSI scores) than those without, and that sexual abuse was a stronger predictor for psychological distress when compared with other individual demographics such as an alcoholic parent or a parent with mental illness. For the female prisoners, the high levels of psychological distress, as well as demographics similar to the research groups described above, suggest that further analysis of the SCL-90 scores would show profiles of mood disturbances or disorders similar to those of psychiatric populations. It may well be that the high levels of psychological distress of female prisoners are also indicative of the diminishing numbers of mental health hospitals and community mental health care facilities in the last 10 years (Henderson et al., 1995). Prisons, with their increasing female population (Bureau of Justice Statistics, 2002), may have become an undefined institution for housing increasing numbers of mentally ill individuals whom may have been treated within the mental health community.

As expected, results of the current research show a relationship between certain levels of family childhood family conflict and expressiveness and levels of dissociation and/or psychological distress. These findings make sense in relation to offender and attachment research that shows how numerous adult attachment/ relationship problems, as well as the development of criminal behavior, are rooted in the same types of restrictive (low) family expressiveness and invasive (high) family conflict (Allen et al., 1996; Farrington, 1995; McCellan et al., 1997; Pianti et al., 1999) that are significantly associated with higher levels of dissociation and psychological distress (Finklehor et al., 1990; Harvey \& 
Herman, 1995; Nash et al., 1993).

As noted previously, for female prisoners, no interaction effect was found between a sexual abuse history and each of the three childhood family relationship dynamics with levels of dissociation and psychological distress. Analysis of mean scores (See Tables 1 through Table 9) for those with and without a sexual abuse history, reveals a consistent pattern of higher levels of dissociation and psychological distress for those with sexual abuse histories and levels of family dynamics that have been defined as detrimental to a survivor's emotional healing (high conflict, low expressiveness, low cohesiveness) (Barrett et al., 1996; Briere \& Elliot, 1993; Long \& Jackson, 1994; Nash et al., 1993). It does seem to appear that when female prisoners with a sexual abuse history also experienced high childhood family conflict, low childhood family expressiveness or low childhood family cohesiveness, they identify higher levels of dissociation, and at times higher levels of psychological distress, than those with no sexual abuse history and the same levels of each of the family dynamics. This seems to suggest a possible interaction for female prisoners, who, for example, experience sexual abuse and medium or high conflict, and show higher levels of dissociation when compared to those with a sexual abuse history and low conflict. The extremely unequal number of individuals within the high conflict group may well be affecting the analysis of interaction.

A more in-depth exploration of the relationship of each of the three childhood family dynamics to levels of dissociation and psychological distress provides some insight to the to understanding the etiologic origin of female prisoners' entrenched symptoms. For example, 98 of the participants, with or without sexual abuse histories, described their childhood families as having high levels of conflict. Within this group, 63 women identified both a sexual abuse history and high family conflict. As noted previously, 
mean DES scores (See Table 1) shows are nearly similar for individuals with a sexual abuse history and medium and high levels of family conflict and those without a sexual abuse history and high family conflict. Medium or high conflict, for these women, regardless of whether they experienced childhood sexual abuse, induced dissociation. Nash et al., (1993) and other researchers have noted that dissociation helps protect against the stress of a highly conflictual family, the lack of emotional support and/or an inability to perceive or express emotions (Inderbitzen-Pisaruk et al., 1992; Long \& Jackson, 1994). Zingman and Boswell (1996) also found, for college students, that conflict alone had significant impact on clinical levels of dissociation even without sexual abuse. It may be that any level of conflict (medium or high) creates and emotional overload for individuals who do not have effective emotional coping tools. This is further supported by findings that medium levels of conflict, when not interacting with the effects of sexual abuse, do not appear to initiate dissociation. It may also be that, for sexual abuse survivors, even medium levels of family conflict compound issues related to the sexual abuse, thus initiating a need to dissociate from trauma cues (Nash et al., 1993; Rosenstein \& Horowitz, 1996; Stuppy, 1996).

In a somewhat contrasting finding, for incarcerated females with a sexual abuse history, all levels of childhood family conflict are related to clinical levels of psychological distress, while only those with high family conflict and no sexual abuse history have clinical levels of psychological distress (see Table 2). In other words, it may be that even the absence (low) of childhood family conflict does not lessen emotional problems indicated by high levels of psychological distress (Derogatis, 1994). Some type of relationship may occur between sexual abuse and conflict that exacerbates psychological problems resulting from childhood sexual abuse but that were also initiated 
by family dynamics prior to the abuse. This is consistent with findings that note that disrupted attachment can predict limited coping skills (Morrow \& Smith, 1995; Pianta et al., 1996).

The afore-mentioned analysis of the relationship of the incarcerated female sexual abuse survivor's level of perceived childhood family conflict in relation to differing levels of dissociation, yet consistently clinical levels of psychological distress, (See Table 1 and Table 2) may represent that dissociation is more sensitive to the current presence or absence of family conflict (Ursano \& Fullerton, 1999; van der Kolk, 1987). Such a hypothesis related to dissociation's sensitivity to family conflict is further supported by the multivariate analysis, which indicated that childhood family conflict's main effect was due to dissociation rather than psychological distress (see Results: Regarding Hypothesis 1). It is suggested that female prisoners with a sexual abuse history and low family conflict (See Table 1), may have had more opportunities within the family to express and work through of emotions, behaviors which have a diminishing effect on dissociation (Nash et al., 1993; Waites, 1993). In contrast, clinical levels of psychological distress for those with childhood sexual abuse and all levels of family conflict (See Table 2), suggests that for female prisoners, psychological distress is more entrenched, possibly associated with a sexual abuse history and not diminished by lack of family conflict. This hypothesis is further supported by the absence of clinical levels of psychological distress for those with no sexual abuse history and low or medium levels of family conflict. Future analysis may also show that levels of psychological distress may also be more closely related to lack of counseling and/or negative experience of disclosure (Gold, 2000; Terr, 1991). Out of the 73 female prisoners who identified a sexual abuse history and disclosure experiences, 45 of these women indicated these 
experiences as harmful. It may well be that lower levels of dissociation for those with a sexual abuse history are indicative of supportive disclosure or counseling experiences (Everill \& Waller, 1994). Data regarding these and other possible confounding variables has not yet been analyzed. Future regression analysis could analyze what additive influences may be occurring.

Analysis of childhood family expressiveness's influence on the female prisoners' levels of dissociation and psychological distress also seems to suggest some type of relationship between sexual abuse and family expressiveness on symptom levels. Out of the 111 female prisoners identifying low childhood family expressiveness, 65 of these women identified a sexual abuse history and corresponding highest levels of dissociation and psychological distress (See Table 4 and Table 5).

In a somewhat contrasting response pattern to that seen with family conflict, only female prisoners with a sexual abuse history and low family expressiveness had clinical levels of dissociation when compared to those both prisoners with a sexual abuse history and medium or high levels of family expressiveness and those without a sexual abuse history and all levels of family expressiveness (see Table 4). For female prisoners with a sexual abuse history, low family expressiveness may denote an inability to emotionally heal, while medium and high levels of expressiveness possibly enhance the process of emotional healing or resiliency against dissociation (Bowlby, 1988; Collins, 1996; Gold et al., 1999; Stuppy, 1996). It is suggested that individuals with a CSA history and low childhood family expressiveness have not been taught and/or allowed to express and work through the varied trauma-related emotions (Fredrickson, 1992; Nash et al., 1993).

Such a high percentage (74\%) of female prisoners identifying families with low expressiveness and clinical levels of dissociation, strongly suggests that low 
expressiveness for female prisoners with a sexual abuse history predicts high levels of dissociation. These are not surprising findings for this population, in that research cited above and previously, notes that low expressiveness is a predictor of offending behavior and part of the demographic profile of female prisoners (Farrington, 1995; Gorsuch, 1998 ) as well as a predictor of higher levels of dissociation for those with sexual abuse histories (Haberstadt et al., 1995; Kluft, 1990). It is suggested that possibly minimal emotional expression and emotional suppression (low levels of family expressiveness) are culturally (offender population) expected dynamics. Prisoner demographics identified by Farrington, (1995), Bureau of Justice Statistics (2000) and McCellan et al., (1997) documented generational histories of family conflict, abuse, personal histories of foster care, and attachment problems in childhood and adulthood. Such demographics would denote environments where little emotional modeling or expression occurs (Sweezy, 1998). If this is true, medium or high levels of family expressiveness may be welcomed and thus diminish the need to dissociate.

In contrast, Zingman and Boswell (1996) found, for college student sexual abuse survivors, only medium levels of childhood family expressiveness were related to nonclinical levels of dissociation. High and low levels of childhood family expressiveness for college students with sexual abuse histories was related to clinical levels of dissociation. The 1996 study suggested that, for college populations, high levels of family expressiveness possibly create an invasive environment that limits or detracts from emotional expression in ways similar to a non-expressive environment. In addition, the Zingman and Boswell study, especially in relation to this female prisoner study, demonstrates how convenience populations often do not provide findings that can be generalized. 
For female prisoners with a sexual abuse history, childhood family expressiveness may not offer curative effects against clinical psychological distress, in the same way medium and high levels of expressiveness seemingly diminish dissociation. Clinical levels of psychological distress are found across all levels of family expressiveness (See Table 5). In addition, for the prisoners who identified no sexual abuse history; only 12 women with high family expressiveness had non-clinical levels of psychological distress. Once again, and similar to family conflict, psychological distress, for female prisoners, may well be more firmly entrenched and less sensitive to the influence of family dynamics. It could be that the compounded difficulties, precipitated by families that predict psychological distress (Pianta et al., 1996), create such entrenched symptoms that only the highest levels of family expressiveness diminish the multi-dimensional symptom responses (Derogatis, 1994).

The inability of family expressiveness, (similar to conflict) to have differing influences on levels of psychological distress for both those with and the majority of those without a sexual abuse history, strongly suggests that, in relation to family expressiveness, for female prisoners, family is more pathogenic than sexual abuse (Gold, 2000; Nash et al., 1993). Interestingly, while all levels of both family expressiveness and family conflict showed clinical levels of psychological distress, expressiveness was the only factor that showed the main effect equally distributed across both dissociation and psychological distress. Thus, it may well be that, for female prisoners, level of family expressiveness is a primary predictor of a highly symptomatic profile (Gillard \& Beck, 1998; Kurshan, 1997).

In conjunction with the above findings, research that has shown that low family expressiveness can predict a high rate of anger and affect intensity (Haberstadt et al., 
1995 ) and that anger has been seen as major emotion regulation issue for offenders (Baunach, 1982, Collins, 1996). This could suggest that the limited anger regulation may also impact the female prisoner's level of psychological distress, as well as anger having a diminishing effect on any curative factors. As noted previously, individuals with high levels of anger can, through their behaviors, deter others from assisting them (Derogatis, 1994). Analysis of GSI subscales for those with high levels of psychological distress may help identify if symptom clusters related to anger, such as hostility, are related to the highest levels of psychological distress. In addition, without further analysis of what levels of family cohesiveness and conflict are found for these female prisoners with the most symptomatic expressiveness psychological distress profile, what influence counseling or disclosure experiences have on psychological distress, it is hard to ferret out the specific influence of family expressiveness influences on levels of psychological distress.

Even though no significant main effect was found for family cohesiveness on levels of dissociation and psychological distress, observed patterns of levels of dissociation and psychological distress across levels of family cohesiveness mirror patterns of family conflict and expressiveness (see Tables 6 and 7). The largest group $(n=89)$ of female prisoners endorsed low levels of family cohesiveness, with 56 of these women identifying a sexual abuse history. Unlike expressiveness, but similar to conflict, the observed means for those with a sexual abuse history and medium levels of cohesion showed a clinical level of dissociation (see Table 7). For sexual abuse survivors only those with high family cohesiveness showed a non-clinical level of dissociation. These observed level patterns of dissociation differ from college students with sexual abuse histories, for whom only medium levels of cohesiveness were related to lower levels of 
dissociation (Zingman \& Boswell, 1996). The rational for why only non-clinical levels of dissociation are observed for female prisoners with a sexual abuse history and endorsing high levels of cohesion may, once again, be found within their demographic profile. As noted previously (Sweezy, 1998), incarcerated females with histories of disrupted and single parent families may experience non-cohesive (low) families as a population norm (see demographic stats). With such a history, even medium levels of family cohesiveness may not be trusted to endure. Confounding factors such as level of family expressiveness and of family conflict, as well as the influence of disclosure and counseling experiences must be analyzed to fully understand cohesiveness's effect dissociation.

While, for female prisoners, no significant main effect was found for family cohesiveness, the observed patterns of levels of psychological distress for cohesiveness mirror the relationships that family conflict and family expressiveness have with psychological distress. For both those who identified a sexual abuse history and those who did not, clinical levels of psychological distress were found for all groups except for those with high family cohesiveness and no sexual abuse history (See Table 8).

Without significant findings of a main effect for cohesiveness, results cannot be truly analyzed. Even so, it is suggested that future research may find that the culture-bound dynamics discussed previously and related to non-cohesive families (McClellan et al., 1997; Sweezy, 1998) increase the multi-layered mental health issues that make up psychological distress. It may also be that only the highest level of family cohesion is trusted or believed to be permanent by when an individual comes from a culture where family cohesion is not a norm (Gorsuch, 1998). In addition, it may be that while medium levels of cohesiveness could provide a sense of safety and trust, levels of family expressiveness and/or conflict, may combine to create barriers to awareness or perception 
of the safety or support that a medium cohesive family provides.

For incarcerated females both with and without a sexual abuse history, the above symptom patterns related to levels of family conflict, expressiveness and cohesiveness, do identify this population as one whose members display entrenched and complicated psychological symptom profiles. Results clearly show that for incarcerated females, dissociation is generally a trauma response based in sexual abuse and complicated by certain levels of family conflict and family expressiveness. The consistently high, clinical levels of psychological distress for those both with and without a sexual abuse history suggest such symptom profiles may be closely affiliated with expected demographic profiles of offenders (Gorsuch, 1998). More specifically, for female prisoners identified as highly dissociative and experiencing high levels of psychological distress, and with offending research noting the historical patterns of dysfunctional family patterns, it is suggested that for this population, family may be more pathogenic than the abuse itself.

It is especially interesting to note for female prisoners with clinical levels of dissociation and psychological distress, the majority of these individuals also endorsed a sexual abuse history and low family cohesiveness, low family expressiveness, and high family conflict. The three specific levels of family relationship dynamics noted above, have been described as predicting limited adult coping skills (Nash et al., 1993) as well as influencing fear-related current and negative future-oriented expectations (MacLeod \& Bryne, 1996; Pianti et al.: 1996). Female prisoners may well embody such fear based, negative cognitions about their future. Such cognitions may be somewhat related to a reciprocal relationship between levels of dissociation and anxiety, especially in relation to past heightened anxiety about the ongoing sexual abuse. At the same time, such anxiety, interacting with the negative family environment, has also been associated with 
orientations of hopelessness and worry, and subsequently, mental defeat (Ehler et al., 2000). The family demographics, noted previously, for the female prisoner population (Gorsuch, 1998) may influence of attitudes of mental defeat and thus further compound psychological problems that make up psychological distress. This could explain why female prisoners with a sexual abuse history and childhood families of high levels of expressiveness and cohesiveness, have clinical levels of psychological distress across all three levels of both family dynamics but not for dissociation. An attitude of mental defeat could increase hopelessness, anxiety, hostility, and interpersonal sensitivity; all factors in increasing psychological distress (Derogatis, 1994)

The difference between the ways levels of dissociation and psychological distress are influenced by the level of each family dynamics may indicate that different aspects of family impact trauma response symptoms in different ways. More specifically for sexual abuse survivors, family with high levels of expressiveness or low conflict may provide some form of emotional support or sense of unity that instills a sense of protection (seen by diminished dissociation). For individuals oriented to mental defeat and hopelessness, childhood families may have diminished any ability to trust any type of support or protection. The high levels of psychological distress may indicate, for this population, that such belief systems of mental defeat have endured into adulthood.

Thus, while this research has found that for incarcerated women, a sexual abuse history does play a crucial role in the etiologic development and persistence of dissociation and psychological distress (Gold, 2000), this research also finds that family conflict and family expressiveness may play a stronger role in the development and maintenance of the female prisoner's multi-dimensional clinical symptom presentation. This confirms Brock et al., (1997) and Finklehor et al. (1990) findings that it is not sexual 
abuse alone but strong mediating factors that play a crucial role in determining symptom level.

If childhood family dynamics, beyond the impact of trauma, are primary mediating factors for levels of dissociation and psychological distress, there may be two explanations for the influence of family dynamics on symptom levels. It could well be that, as noted previously, such family dynamics limit an in individual's ability to cope with emotional trauma and modulate and tolerate the related emotions. In addition, it may be that the change in the intensity of dissociation or psychological distress is not due to the family dynamics adding to the trauma effects but rather that when sexual abuse occurs, the impact of such an invasive trauma, diminishes an even minimal restorative effect that a family dynamic which is organized around dysfunctional dynamics, might provide for individuals without a sexual abuse history. It appears that sexual abuse actually reinforces the messages, belief systems, and rules of the dysfunctional female prisoner's childhood family.

In addition, and in relation to the above analysis of all family dynamics, for female prisoners with a sexual abuse history, the high percentage of clinical levels of psychological distress and dissociation may indicate the initial identification of some sort of reciprocal relationship between dissociation and psychological distress. It may also be that the prison environment itself affects such a relationship.

\section{Suggestion for future research}

The analysis and interpretation of results also raised a number of questions that could be addressed by future research.

- Will other highly symptomatic and/or under-researched populations show similar 
relationships between levels of family conflict, expressiveness and cohesiveness with levels of dissociation and psychological distress?

- Are the same population demographics that influence female prisoners' high levels of dissociation and psychological distress specific to incarcerated females or specific to the most symptomatic sexual abuse survivor population?

- Is the incarcerated female CSA population the most symptomatic of underresearched and untreated CSA populations?

- Do individuals with a sexual abuse history and the combination of high family conflict, low family expressiveness and low family cohesiveness have the highest levels of dissociation and psychological distress?

- Are specific symptom subscales on the GSI related to the highest levels of psychological distress? Specifically are problematic and clinical levels of interpersonal sensitivity, somaticism, and hostility related to higher levels of psychological distress?

- Do levels of dissociation and psychological distress diminish when female prisoners are released?

- What impact do disclosure and counseling experiences have on levels of dissociation or psychological distress?

- In relation to current findings, does time-period of occurrence of sexual abuse (i.e. during the first third of childhood, during first and second third of childhood, etc.) predict higher levels of dissociation or psychological distress?

- In relation to current findings, does relationship of the perpetrator to the sexual 
abuse survivor predict higher levels of dissociation and psychological distress?

\section{$\underline{\text { Summary }}$}

The analysis of symptom presentations of incarcerated female sexual abuse survivors, confirmed some sexual abuse-trauma response theories based on convenience populations and helped develop new hypothesis regarding the coexistence of dissociation and levels of psychological distress. Most importantly, these findings identify female prisoners as a highly symptomatic yet untreated sexual abuse survivor population.

For incarcerated females, dysfunctional childhood family relationship dynamics of conflict and expressiveness do impact dissociation magnitude and levels of psychological distress. For female prisoners with a sexual abuse history, the highest clinical levels of dissociation were significantly associated to high conflict and low expressiveness. Levels of clinical psychological distress, for prisoners with a sexual abuse history, were associated with all levels of family conflict and family expressiveness. For female prisoners without a sexual abuse history, no clinical levels of dissociation were associated with family expressiveness while high family conflict was associated with clinical levels of dissociation. In relation to psychological distress and no history of sexual abuse, only female prisoners with high family conflict had clinical levels of psychological distress, while those with both low and medium levels of family expressiveness had clinical levels of psychological distress.

Such findings appear to confirm Bryer et al., (1986) and Chu \& Dill's (1990) assumption that trauma alone is not the primary predictor of greater dissociative responses or other trauma-symptom development. In addition, this research supports Williams (1994) and Gold (2000) suggestion that many under-treated female 
sexual abuse survivors have extensive histories of social and dehabilitating mental health issues. This appears true for this incarcerated female sexual abuse survivor population, with more than $57 \%$ of females endorsing a sexual abuse history as well as high levels of dissociation and psychological distress. The female prison population may well be the most symptomatic of all sexual abuse survivor populations. Conversely, while prisoners, as a specific population, may not represent the most symptomatic of sexual abuse survivors, it may well be that the culture bound demographics of the female offender population will be found in the majority of highly symptomatic CSA populations.

This study has both theoretical and practical applications for both general and specific sexual abuse survivor populations. When one looks at the disproportionate number of women in prison who have multi-generational abuse histories and multi-generational incarceration histories within their families, trauma research with this population becomes very important. With symptom presentations similar to highly symptomatic clinical populations, it seems imperative that more effective interventions must be developed for female priosners (Gold et al., 1999). Data from this and future replication studies can be applied to the development of more effective interventions. Such interventions could integrate therapeutic and psycho-educational programs that both treat, and educate this population on the effect of both childhood sexual abuse and of the researched family dynamics' impact on emotional development and healing.

Specifically, data related to how family conflict, expressiveness, and cohesiveness affect coping abilities can be applied in ways that provide restorative factors that the prisoner's childhood families did not offer. This could benefit the incarcerated female sexual abuse survivor, by initiating change in the structure of negative core beliefs about 
self, about emotional expression and self efficacy that have been impacted by generational patterns of abuse and incarceration. In that offending behavior also has its roots in similar etiologic origins as the described debilitating trauma response symptoms (Gorsuch, 1998) such interventions could also begin to diminish offender behavior and attitudes, while possibly interrupting the dysfunctional coping patterns that female prisoners could pass down to their children (Farrington, 1995).

Finally, while this study has identified a previously unrecognized symptomatic and untreated sexual abuse survivor population, and had a more diverse cultural representation than research with clinical or college populations, the sample was not large enough to make any generalizations about sexual abuse survivors from non- Caucasian cultures. Analysis of similarities and differences between sexual abuse survivors of different cultural groups could be replicated through comparison studies of African American, Hispanic, Asian, Native American, and other cultural groups.

This study in general has begun to answer questions about under-researched sexual abuse survivor populations but more so, it opens the door to many more questions about why certain populations are more symptomatic than others. Numerous replication and comparison studies could be designed to begin to answer some of these questions. 


\section{REFERENCES}

Ainsworth, M. \& Eichberg, C. (1991). Effects on infant-mother attachment of mothers unresolved loss of an attachment figure, or other truanmtic experience. In C. M. Parkes, J. Stevenson-Hinde \& P. Marris (Eds.), Attachment Across the Life Cycle (160-163). London: Routledge-Kegan Paul.

Alexander, P. C. \& Anderson, C. L. (1994). An attachment approach to psychotherapy with the incest survivor. Psychotherapy, 31, 185-195.

Allen, J. P., Hauser, S. T., \& Borman-Spurrel, E. (1996). Attachment theory as a framework for understanding sequela of severe adolescent psychopathology: an 11year follow-up study. Journal of Consulting and Clinical Psychology 64, 254-263

American Psychiatric Association (1984). Diagnostic and Statistical Manual of Mental Disorders. (4th ed.). Washington, D. C.: Author.

Barker-Collo, S. (2001). Relationship of the dissociative experiences scale to demographics, symptomology and coping strategies in a New Zealand student sample. Journal of Trauma and Dissociation, 2, 81- 101.

Barret, P. M., Dadds, P. M., Dadds, M. R. \& Rapee, R. M. (1996). Family treatment of childhood anxiety: a controlled trial. Journal of Consulting and Clinical Psychology, 64, 333-342.

Baunach, P. J. (1982). You can't be a mother and be in prison ... can you? Impacts of mother-child separation. In Price, B. \& Sokoloff, N (eds.) The Criminal Justice System and Women pp. New York: Clark Boardman Ltd.

Benningfield, M. F. (1992). The use of hypnosis in treatment of dissociative patients. Journal of Child Sexual Abuse, 1, 17-31 
Berkowitz, A. \& Perkins, H. W. (1988). Personality characteristics of children of alcoholics. Journal of Consulting and Clinical Psychology, 56, 206-209.

Bloch, E. L. (1991). Post-traumatic stress disorder: Therapeutic approachavoidance: an illustrative case. Psychotherapy, 28, 162-167

Boney-McCoy, S. \& Finklehor, D. (1996). Is youth victimization related to trauma symptoms and depression after controlling for prior symptom and family relationships? A longitudinal prospective study. Journal of Consulting and Clinical Psychology 64, $1406-1416$.

Bowlby, J. (1988). A secure base: Parent-child attachments and healthy human development. New York: Basic Books.

Braun, B. G. (1986). Issues in psychotherapy of multiple personality disorder. In Braun, B. G. Treatment of Multiple Personality Disorder. (pp. 128):Washington, D. C.: American Psychiatric Press.

Briere, J. \& Elliot, D. M. (1993). Sexual abuse, family functioning and psychological symptoms: on the validity of statistical control. Journal of Consulting and Clinical Psychology 61, 284-288.

Briere \& Runtz (1988). Symptomatology associated with childhood sexual victimization in a nonclinical adult sample. Child Abuse and Neglect, 12, 51-59.

Brock, K. J., Mintz, L. B. and Good, G. E. (1997). Differences among sexually abused and nonabused women from functional and dysfunctional families. $\underline{\text { Journal }}$ of Counseling Psychology, 44, 339-345.

Brown, A. and Finklehor, D. (1986). Impact of child sexual abuse: a review of the literature. Psychological Bulletin, 99, 66-77. 
Bryer, J. B., Nelson, B. A., Miller, J. B. \& Krol, P. A. (1987). Childhood sexual and physical abuse as factors in adult psychiatric illness. American Journal of Psychiatry, 144, 1426-1430.

Burkett, L. P. (1991). Parenting behaviors of women who were sexually abused as children in their families of origin. The Family Process, 30, 421-433.

Carlson, E. B., \& Putnam, F. W. (1993). An update on the Dissociative Experiences Scale. Dissociation (Form), 6, 16-27.

Chu, J. A. and Dill, D. L. (1990). Dissociative symptoms in relation to childhood phyyical and sexual abuse, American Journal of Psychiatry, 147, 887893.

Cole-Detke, H. \& Kobak, (1996). Attachment processes in eating disorder and depression. Journal of Consulting and Clinical Psychology 64, 291-294.

Collings, S. J. (1994). The long-term effects of contact and non-contact forms of child sexual abuse in a sample of university men. Child Abuse and Neglect, 19, 1-6.

Collins, N. L. (1996). Working models of attachment: Implications for explanation, emotion and behavior. Journal of Personality and Social Psychology, $\underline{71}, 810-832$.

Collins, F. E. \& Ffrench, C. H. (1998). Dissociation, coping and locus of control in a non-clinical population: Clinical implications. Australian Journal of Clinical and Experimental Hypnosis, 26, 113 - 126.

Coyne, J. C. \& Schwenk, F. (1997). The relationship of distress to mood disorders in primary care and psychiatric populations. Journal of Consulting and Clinical Psychology, 65, 161-168. 
Courtois C. A. (1988). Healing the Incest Wound: Adult survivors in therapy. New York: W. W. Norton.

Cronkite, R., Finney, J., Nekich, J. \& Moos, R. (1990). Remission among alcoholic paitents and family adaptation to alcoholism: a stress and coping perspective. In R. L. Collins, K. E. Leonard, \& J. Searles (Eds.) Alcohol and the Family: Research and Clinical Perspectives (309-307). New York: Guilford Press.

DeKylen, M. (1996). Disurptive behavior disorder and intergenrational attachment patterns: a comparison of clinic-referred and normally fiunctioning preschoolers and their mothers. Journal of Consulting and Clinical Psychology, 64, $357-365$.

Derogatis, L. (1994). SCL-90-R Administration, scoring and procedures manual-third edition. Minneapolis: National Computer Systems.

Derogatis, L. and Lazarus, L. (1994). The SCL-90, Brief Symptom Inventory and matching clinical scales. In M.E. Marush (Ed.) The use of psychological testing in treatment planning and outcome assessment (pp 217-248). Hillsdale, NJ

Dubester, K. A. \& Braun, B. G. (1995). Psychometric properties of the dissociative experience scale. Journal of Nervous and Mental Diseases, 183, 231235.

Dutton, M. A. (1994). Post-Traumatic therapy with domestic violence survivors. In Williams, M. B., and Sommer, J. F. (Eds.), Handbook of PostTraumatic Therapy, pp. 146-161.

Dutton, D. G. \& Hemphill, K. J. (1992). Patterns of socially desirable responding among perpetrators and victims of wife assault. Violence and Victims, 
Z (1), 29-39.

Ehlers, A., Maercker, A., Boos, A. (2000). Posttraumatic stress disorder following political imriosnment: the role of mental defeat, alienation, and perceieved permannet change. Journal of Abnormal Psychology, 109, 45-55.

Ellason, J. W. \& Ross, C. A. (1995). Positive and negative symptoms in dissociative identity disorder and schizophrenia: A comparative analysis. The Journal of Nervous and Mental Disease, 183, 236-241.

Everill, J. \& Waller, G. (1994). Disclosure of sexual abuse and psychological adjustment in female undergraduates. Child Abuse and Neglect, 19, 93-100.

Everstine, D. S. \& Everstine, L. (1993). The Trauma Response: Treatment for Emotional Injury. New York: W. W. Norton \& Co.

Farrington, D. P. (1995). The development of offending and antisocial behavior from childhood: Key findings from the Cambridge study in delinquent behavior. Journal of Child Psychology and Psychiatry, 360, 929-964.

Finkelhor, D., Hotaling, G., Lewis, L. A. \& Smith, C. (1990). Sexual abuse in a national survey of adult men and women; prevalence, characteristics and risk factors. Child Abuse and Neglect, 14, 19-28.

Flannagan, P. J. (1998). Differential treatment of the female offender in oklahoma: an exploratiry study of boundary maintenance throery. Dissertation Abstracts International Section A: Humanities and Social Sciences, 59, 0618.

Ford, D., Fisher, J. P. \& Larson, L (1997). Object relations as a predictor of treatment outcome with chronic posttraumatic stress disorder. Journal of Consulting and Clinical Psychology 1997, 65, 547-559. 
Forward, S. \& Buck, C. (1978). Betrayal of innocence and its devastation. New York: Penguin books.

Fredrickson, R. (1992). Repressed memories: a journey to recovery from sexual abuse. New York: Simon and Schuster.

Gold, S. R. (2000). Not trauma alone: therapy for child abuse survivors in family and social context. Philedeiphia: Brunner-Routledge.

Gold, S. R., Milan, L. D., Mayall, A. \& Johnson, A. E. (1994). A crossvalidation study of the trauma symptom checklist: The role of mediating variables. Journal of Interpersonal Violence, 9 , 12-26.

Gold, S. N., Hill, E. L., Swingle, J. M. \& Elfant, A. S. (1999). Relationship between childhood sexual abuse characteristics and dissociation among women in therapy. Journal of Family Violence, 14, 157-171.

Gorsuch, N. (1998). Unmet need among disturbed female offenders. Journal of Forensic Psychiatry 9, 556-570.

Greaves G. B. (1993). A history of MPD. In Kluft, R. P. (eds.), Clinical Perspectives Multiple Personality Disorder (pp. 355-380). Washington D. C.: American Psychiatric Press.

Greenberg, M. T., Speltz, M. \& Deklyen, M. (1993). The role of attachment in the early development of disruptive behavior problems. Development and Psychopathology, 5, 191-214.

Greenfield, L. A, \& Minor-Harper, S. (1991) Women in prison. Bureau of Justice Statistics Special Report.

Haberstadt, A. G., Cassidy, J., Stiffer, C. A., Parke, R. D. \& Fox, N. A. (1995) 
Self-expressiveness within the family context: Psychometric support for a new measure. Psychological Assessment, 7, 93-103.

Harvey, M. R. \& Herman, J., L. (1994). Amnesia, partial amnesia and delayed recall among adult survivors of childhood trauma. Consciousness and Cognition, 3 , 295-306.

Heatherton, T. F. \& Baumeister, R. F. (1991). Binge eating as escape from self-awareness. Psychological Bulletin, 110, 86-108.

Henderson, D., Schaeffer, L. B. \& Brown, L. (1995) Gender-appropriate mental health services for incarcerated women: issues and challenges. Family and Community Health, 21, 42-53.

Herman, J. L. (1992). Complex PTSD: a syndrome in survivors of prolonged and repeated trauma. Journal of Traumatic Stress, 5, 377-391

Inderbitzen-Pisaruk, H., Shawchuck, C. R. \& Hoier, T. S. (1992). Behavioral characteristics of child victims of sexual abuse: A comparison study. Journal of Clinical Child Psychology, 21, 14-19.

Kemp, M. A. and Neimeyer, G. J. (1999). Interpersonal attachment: experiencing, expressing, and coping with stress. Journal of Counseling Psychology, $\underline{46}, 388-394$.

Kessler, B. L. and Bieschke, K. J. (1999). A retrospective analysis of shame, dissociation and adult victimization in survivors of childhood sexual abuse. Journal of Counseling Psychology, 46,335-341.

Kluft, R. P. (1982). Various interventions in the treatment of multiple personality disorder. American Journal of Clinical Hypnosis, 24, 230-240. 
Kluft, R. P. (1986) Personality unification in multiple personality disorder: A follow up study. In Braun, B. G. (Ed.). Treatment of Multiple Personality Disorder. (pp. 29-60). Washington, D. C.: American Psychiatric Press.

Kluft R. (1990). Dissociation and subsequent vulnerability; a preliminary study. Dissociation, 3,167-173.

Kluft, R. P. (1994) Counter transference in the treatment of multiple personality disorder. In Wilson, J. P., \& Lindy, J. D., (Eds.). Counter-Transference in the Treatment of PTSD (pp. 122-150). Guilford Press: New York.

Koraleski, S. F., \& Larson, L. M. (1997) A partial test of the transtheoretical model in therapy with adult survivors of sexual abuse. Journal of Counseling Psychology. 44, 302-306.

Liotti, G. (1992). Disorganized/disoriented attachment in the etiology of the dissociative disorders. Dissociation, 5, 196-204.

Long, P. J., \& Jackson, J. L. (1994). Childhood Sexual abuse: An examination of family functioning. Journal of Interpersonal Violence, 9, 270-277.

Lynn, S. \& Rhue, J. (1994). Dissociation : clinical and theoretical perspectives. New York: Guilford Press

MacLeod, A. K. \& Bryne, A. (1996). Anxiety, depression and the anticipation of future positive and negative experiences. Journal of Abnormal Psychology, 105, 286-289.

Malinosky-Rummel, R. R. \& Hoier, T. S. ( 1992). Validating measures in sexually abused and non-abused children. Psychological Assessment, 13, 341-357.

Mallinckrodt, B., McCreary, B. A. \& Robertson, A. K. (1995). Co-occurrence of 
eating disorders and incest: The role of attachment, family environment and social competencies. Journal of Consulting Psychology, 42, 178-186.

McClellan, D. S., Farabee, D., \& Crouch, B.M.(1997). Early victimization, drug use, and criminality: A comparison of male and female prisoners. Criminal Justice and Behavior, 24, 455-476.

Moos, R. H. \& Moos, B. S. (1986). Family Environment Scale Manual. (2nd Ed.) Palo Alto, CA: Consulting Psychologists Press.

Moos, R. H. \& Moos, B. S. (2002). Family Environment Scale Manual. (3rd Ed.) Palo Alto, CA: Stanford University Medical Center.

Morgan, E., (1996). Types of dissociative behaviors observed in an urban jail;25 months of participant observation. Dissociation 9, 89-97.

Morrow, S. L. \& Smith, M. L. (1995). Constructions of survival and coping by women who have survived childhood sexual abuse. Journal of Counseling Psychology, 42, 24-33.

Nash, M., R., Hulsey, T. L., Sexton, M. C., Harralson, T. L., \& Lambert, W. (1993). Long-term sequela of childhood sexual abuse: Perceived family environment, psychopathology, and dissociation. Journal of Consulting and Clinical Psychology, 61, 276-283.

Newman, E., Riggs, D., \& Roth, S. (1997). Thematic resolution, PTSD and complex PTSD: the relationship betwen meaning and truama-related diagnosis. Journal of Traumatic Stress, 10, 197-213.

Nigg, J. T., Lohr, N. E., Westen, D., Gold, L. J. \& Silk, K. R. (1992). Malevolent object representations in borderline personality disorder and major depression. 
Journal of Abnormal Psychology, 101, 61-67.

Nishith, P., Mechanic, M. B., and Resick, P. A. (2000) Prior interpersonal trauma: The contribution to current PTSD symptoms in female rape victims. Journal of Abnormal psychology. 109, 20-25

North, C. S., Ryall, J. M., Ricci, D. A., \& Wetzel, R. D. (1993 ).Multiple personalities, Multiple Disorders; Psychiatric Classification and Media Influence, New York: Oxford University Press.

Oklahoma State Correctional Institute (1995). Mabel Bassett, Kate Barnard and Tulsa Community Correctional Center Demographic Data Summary. (Not in print).

Parker, G., Tupling, H., \& Brown, L. B. (1979). A parental bonding instrument. British Journal of Medical Psychology, 52, 1-10.

Pianta, R. C., Egeland, B., \& Adam, E. K. (1996). Adult attachment classification and self-reported psychiatric symptomology as assessed by the Minnesota Multiphasic Personality Inventory-2. Journal of Consulting and Clinical Psychology, 64, 273-281.

Porter, J. Wilson, C.; \& Frisch, G. R. (1994) Psychotherapy and symptom change. Psychological Reports, 75, 1313-1314.

Putnam, F. W. (1986). Treatment of multiple personality: state of the art. In Braun, B. G. (eds.)Treatment of Multiple Personality Disorder.(pp. 175- 198). Washington, D. C. American Psychiatric Press.

Rhue, J. W. and Lynn, S. J. 1987. Fantasy Proneness and Psychopathology. Journal of Personality and Social Psychology 53: 327-336. 
Root, M. P., Fallon, P. \& Fredrich, W. N. (1985). Bulimia: A Systems Approach to Treatment. New York: W. W. Norton.

Rose, M. (1996) Personality configurations and adult attachment styles as moderators of the relationship between sexual abuse and adult depression among incarcerated and college women. Dissertation Abstracts International 57, 4077

Rosenstein, D. S. \& Horowitz, H. A. (1996). Adolescent attachment and pathology. Journal of Consulting and Clinical Psychology. 64, 244-253.

Ross, C. A., Anderson, G., Fleisher, W. P., \& Norton, G. R. (1992). Dissociative experiences among psychiatric inpatients. General Hospital Psychiatry. 14, 350-354.

Roth, S. Newman, E. Pelcovitz, D. van der Kolk, B. A. \& MANdel, F. S. (1997). Complex PTSD in victims exposed to sexual and/or physiocal abuse: results from the DSM-IV field trial for posttraumatic stress disorder. Journal of Traumatic Stress, 10, 539-556.

Ruscio, A. M., Borkovac, T. D., \& Ruscio, J (2001). Investigation of the structure of worry. Journal of Abnormal Psychology, 110, 413-422.

Rybicki, D. J., Lepowsky, C. M., \& Anndt, S. (1989). An empirical assessment of bulimic patients using multiple measures. Addictive Behaviors, 14, 249-260.

Sandberg, D., Lynn, S. J. \& Green, J. P. (1994). Sexual abuse and revictimization: Mastery, dysfunctional learning and dissociation. In Lynn, S. J. and Rhue, J. W. (Eds.). Dissociation: Clinical and Theoretical Perspectives (pp. 242-267). New York: The Guilford Press.

Speigel, D. (1986). Dissociation, double binds and posttraumatic stress in multiple personality disorder. In Braun, B. G. (Eds.), Treatment of Multiple 
Personality Disorder (pp.61-78). Washington D.C.: American Psychiatric Press.

Spira, J. L (1996). Treating dissociative identity disorder. San Francisco: JosseyBass Publishers.

Spurrel, M. T. \& McFarlane. Post-traumatic stress, dissociation and coping after a natural disaster. Social psychology and psychiatric epidemicology, 28, 194-200

Steinberg, S., Tobin, D., \& Johnson, C. (1990). The role of bulimic behaviors in affect regulation: Different functions for different patient sub-groups? The International Journal of Eating Disorders,9, 51-55.

Stuppy, L. J. (1996). The relationship between dissociation and object-relations impairment in adult female incest survivors with and without personality splitting. Dissertation Abstracts International, 57, 3425.

Sutker, P. B., \& Allain, A. N. (1995). Psychological assessment of aviators captured in W. W. II. Psychological Assessment, 7, 66-67.

Sweezy, M. (1998). Trauma and recidivism: What's guilt got to do with it? Dissertation Abstracts, 58, 4827.

Swett, C., \& Halpert, M. (1993). Reported history of physical and sexual abuse in relation to dissociation and other symptomology in women psychiatric patients. Journal of Interpersonal Violence, 8, 545-555.

Terr, L. (1994). Unchained memories: True stories of traumatic memories, lost and found. New York: Basic Books.

Terr, Lenore (1991). Childhood trauma: outline and overview. American Journal of Psychiatry 148, 10-20.

Todd, D. M.; Deane, F. P., \& McKenna, P. A. (1997). Appropriateness of the 
SCL-90-R adult norms for outpatient and non-patient college samples. $\underline{\text { Journal of }}$ Counseling Psychology 44, 294-301.

Totem, M. S. (1986). Eating disorders in patients with MPD. In Kluft, R. P. (Eds.). Clinical Perspectives on Multiple Personality Disorder (pp. 343-353). Washington D. C.: American Psychiatric Press.

Ursano, Robert J., and Fullerton, M. (1999). Cognitive and behavioral response to trauma. Journal of Applied Psychology, 20, 1766-1776.

United States Bureau of Justice Statistics Report (2003). Annual report, Washington D.C.: Bureau of Justice.

van der Kolk, B. A. (1987). Psychological Trauma. Washington, D. C.: American Psychiatric Press, Inc.

van der Kolk, B. A., van der Hart, 0., \& Marmar, C. R. (1994). Dissociation and information processing in posttraumatic stress disorder. In van der Kolk, B. A., McFarlane, A. C. \& Weisaeth, L. (Eds.) Traumatic stress: The effects of overwhelming experience on mind, body and society (pp. 303-327). New York: Guilford Press.

Waites, (1993). Trauma and survival; post traumatic and dissociative disorders in women. New York: W. W. Norton and CO.

Walker, L. E. (1991). Post-traumatic stress disorder in women: Diagnosis and treatment of battered women syndrome. Psychotherapy, 28, 21-29.

Williams, M. B. (1994). Establishing safety in survivors of severe sexual abuse. In Williams, M. B., and Sommer, J. F. (Eds.). Handbook of Post-Traumatic Therapy (pp. 162-178). 
Wilsnack, S. C., Vogeltanz, N. D., Klassen, A. D. \& Harris, T. R. (1996). Childhood sexual abuse and women's substance abuse: National survey findings. Journal of Studies on Alcohol, 58, 264-271.

Wilson, J. P., (1994a). The need for an integrative theory of post-traumatic stress disorder. In Williams, M. B., and Sommer, J. F. (Eds.). Handbook of Post Traumatic Therapy (pp. 3-18).

Wilson, J. P., \& Lindy, J. D., ed. (1994b). Counter transference in the treatment of PTSD. New York: Guilford Press.

Zingman, M.L. \& Boswell, D. (1996). Childhood sexual abuse and dissociation: Impact of family functioning. Unpublished master's thesis, Oklahoma State University, Stillwater, OK. 
APPENDIX A 


\section{Background Information Sheet}

(Zingman. 2003)

(Please place an $\mathrm{X}$ or number $(1,2,3, \mathrm{etc}$ ) in the space beside your choice unless directed to give other information.)

1) Age

2) Number of times in prison (different sentences):

3) Are you: Married Divorced Widowed Single

4) Ethnic Background (If mixed background, please mark (1) for the one you identify with or (!) if you recognize two cultures as primary. Mark (2) for lesser identification.)

Native American (which tribe) African American

Caucasian Hispanic (which country)

Asian (which country)

Other

5) Who raised you from birth until age 18 ? Please break down into different years if this changed. (Example: mother, birth to 6 years old, grandmother, 7 years old to 13 years old, foster mother, 13 years old etc)

6) Have other family members been in prison? If more than one aunt, uncle etc, please put how many different ones (example: Sister(s) 2 , uncle(s) $\underline{3}$ )

Mother Father Sister(s) Brother(s)

Grandfather (father's side) ___ (mother's side)_ Grandmother(father's side) _ (mother's side) Uncle(s)(father's side) ___ (mother's side) Aunt(s) (father's side)___ (mother's side)

Female Cousin(s) (father's side) ___(mother's side)

Male Cousin(s) (father's side)___ (mother's side)

Other (please describe: example: Step father, foster parent)

\section{Continue to next page}




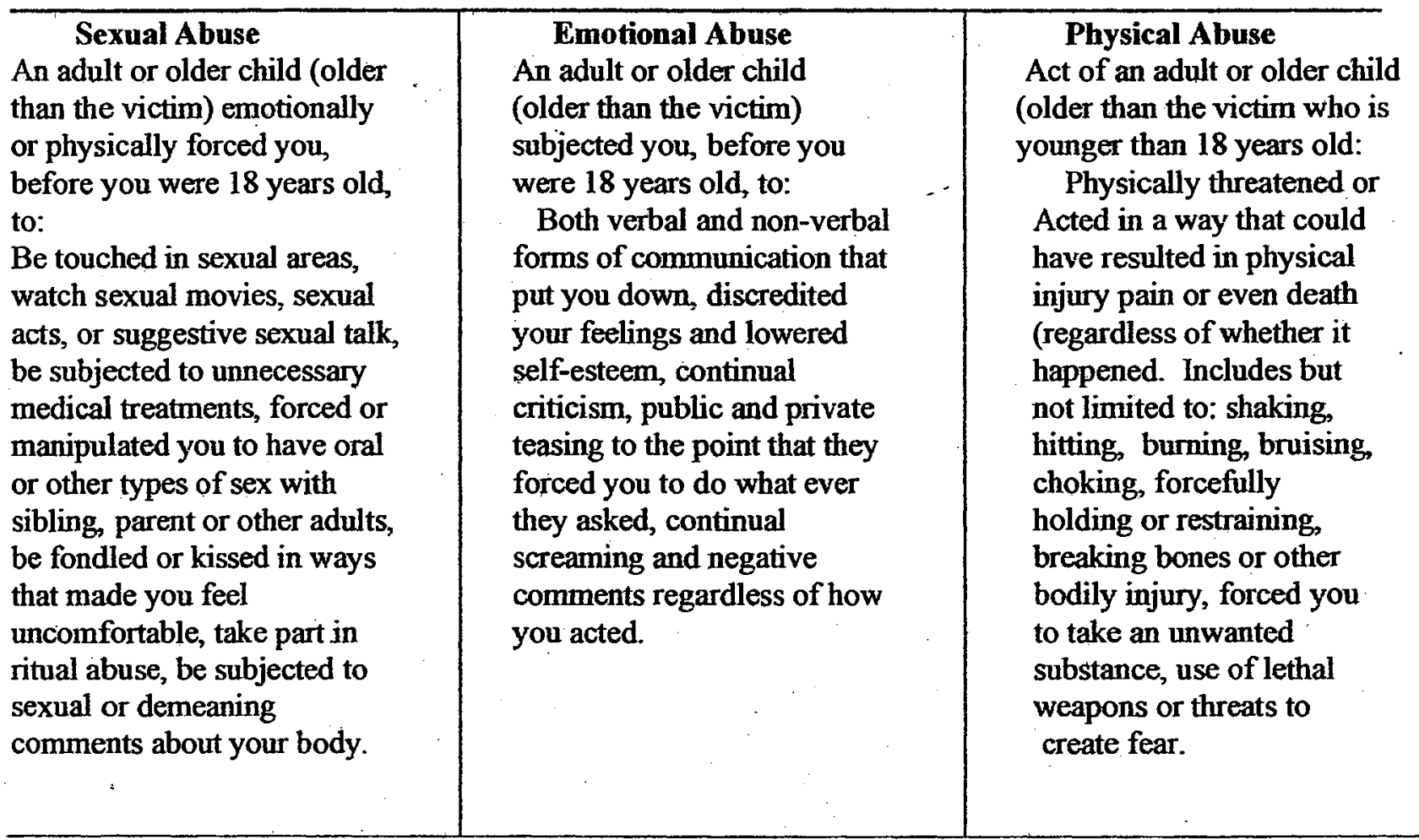

In relation to the above definitions, please answer the next questions.

7) Were you abused from birth through 7 years old? What ages did this occur ( 2 years old until 5 years old, 3 years old, etc)?

Type of abuse (Mark all that occurred.) Please identify with a (1) if only one type occurred or if two types occurred equally. Mark 2 for the type that occurred less often

Sexual abuse (S) ___ Emotional abuse (E)___ Physical abuse (P)

How often did this occur? (Please identify which type of abuse if more than one)

(S - sexual, E - emotional, P - physical)

Every day twice to five times a week once a week

once to three times a month once every two to four months

once or twice a year once a year One time only

8) Were you abused from 8 years old through 12 years old? Yes no What ages did this occur (8 years old until 10 years old, 12 years old, etc)?

Type of abuse (Mark all that occurred.) Please identify with a (1) if only one type occurred or if two types occurred equally. Mark 2 for the type that occurred less often.

Sexual abuse (S) Emotional abuse (E) Physical abuse (P)

\section{Continue to next page.}


Information Sheet

( 8 years old to 12 con't)

Page Three

How often did this occur? (Please identify which type of abuse if more than one)

(S - sexual, E - emotional, P - physical)

Every day twice to five times a week once a week

once to three times a month once every two to four months

once or twice a year once a year One time only

9) Were you abused from 13 years old through 18 years old yes no

What ages did this occur (13 until 15 years old, 14, 15 until 18 years old, etc)?

Type of abuse (Mark all that occurred.) Please identify with a (1) if only one type occurred or if two types occurred equally. Mark 2 for the type that occurred less often

Sexual abuse (S) Emotional abuse (E) Physical abuse (P)

How often did this occur? (Please identify which type of abuse if more than one) ( $S$ - sexual, $E$ - emotional, $P$ - physical)

Every day twice to five times a week once a week

once to three times a month once every two to four months

once or twice a year once a year One time only

10) My abuser was my: (If more than one, please put (1) for either the main abuser or if both abused you equally or (2) for less than the primary abuser)

Mother Father Sister Brother

Aunt (father's side) (Mother's side) Uncle (father's side) (mother's side)

Female Cousin(father's side)___ (mother's side)__ Male Cousin(father's side)__ (mother's side) Grandfather (father's side) (mother's side) Grandmother (father's side) (mother's side)

Other (please describe: example: step father, foster parent, stranger etc )

11) Did any other troublesome or threatening events occur at the same time as the abuse? (Example- death of loved one, divorce of parents, move from familiar home, major injury or sickness of self or family member, etc.)

12) Did you ever tell anyone about the abuse? Yes No If yes: Who

13) Did you feel their response was: helpful or harmful

14) Did they blame you? Yes No

15) Did you get counseling after the abuse? Yes No END OF BACKGROUND INFORMATION SHEET 
APPENDIX B 
Informed Consent / Consent Form

I (name) understand that I am volunteering to participate in a research project looking at how childhood events and family relationships affect an individual's adult personality and coping style. I understand I am being asked to fill in four different questionnaires that contain questions about my family history, major life events, history of abuse, my mental health history and ways that I currently cope with any emotional stress or mental health symptoms. I realize not all questions may relate to my own personal history. I also understand that this study's results may help in developing more effective mental health interventions for both prisoners and the community at large. I have been told that this study is sponsored by Oklahoma State University's Applied Health and Educational Psychology Program and is being conducted by Margaret Zingman, MS, a doctoral student and Donald Boswell, Ph.D., her program advisor. I realize that Ms. Zingman will oversee each 1 and $1 / 2$ hour research session and has an assistant to help distribute and gather paperwork (agreed upon by DOC). I understand that, other than EWCC staff help with gaining access to rooms used for the sessions, no EWCC staff will be involved with the administration of this research.

I understand that my answers are confidential and answer sheets are identified by a participant number only. Although I sign this consent form, this form will be kept separate from my answer sheets. I have been told that the Oklahoma Department of Corrections administration has requested that at least three randomly chosen samples of the consent forms (not answer sheets) be sent to their office for verification purposes, but that neither they nor other correctional staff will be able to view or identify my answer sheets. If I do not want my consent form to be part of the group that samples are chosen from, I can either withdraw from the research or mark the box at the bottom of this form so it will not be placed in the sample group. I do realize that DOC must view some samples for the research to be conducted. I also understand that because I am a volunteer, I can withdraw from the study at any time without penalty. I need only return my packet to the research monitor.

I understand there are no physical risks involved with participation in this study, but that some questions about past family history, closeness of family members, abuse history and my mental health could bring up strong emotions. I understand that Ms. Zingman will use 1-1/2 hours after this and each session to briefly talk with anyone about such issues and, if needed, provide referrals to EWCC Mental Health Services staff. I also understand that, per EWCC rules, I can make a request to staff to Mental Health Services for ongoing counseling. I understand, if distressed, I can also choose to not have my identity remain confidential and mark the back page of the test packet. This page states I need an immediate referral to EWCC mental health staff. There will be no other way for Ms. Zingman to identify my test packet or my distress. I understand this research has been reviewed by the Institutional Review Board of Oklahoma State University, an independent committee (mandated by federal regulations) to review and assess the balance between risk and benefits for subjects of university research. If I want to find out more information on research subjects' rights I can call the IRB contact, Sharon Bacher at 405-744 57.00. If I am concerned about the impact of the research on myself, I can contact mental health services, a prisoner advocate or Margaret Zingman, through the main office of Applied Health and Educational Psychology, Oklahoma State University, Willard Hall, Stillwater, OK, $74075,405-744-5000$.

DO NOT USE AS A SAMPLE TO BE SENT TO THE DOC ADMINISTRATION

I have read and fully understand the consent form and freely agree to take part in this study. I understand I am receiving a copy of this consent form at the end of the session.

Participant

Investigator
Date

Date 
APPENDIX C 


\section{SAMPLE ADVERTISEMENT}

\section{LOOKNNG FOR RESEARCH VOLUNTEERS}

I am currently looking for female prịsoner research volunteers, 18 years and older, to take part in a study investigating the way childhood events and family relationships affect adult personality and coping styles. Your participation will involve answering four different questionnaires that contain questions about family history, major life events, the ways your childhood family communicated, history of abuse, mental health history and ways you currently cope with emotional stress or mental health symptoms.

The questionnaires are written so that those with a $7^{\text {th }}$ or $8^{\text {th }}$ grade reading level should not have too much difficulty reading and answering the questions. All answers will be confidential and no participant will be able to be identified by their answer sheet. Because this is voluntary, no awards or credit will be given for participation. This research is being run under the supervision of Oklahoma State University and with the permission of DOC and EWCC. This is not a Mental Health Services program.

Sessions will occur on Friday, Feb. 14 and Feb.21 and Saturday Feb. 15 and Feb.22 in the Education building and will last for 1 and $1 / 2$ hours. The same amount of time will be provided after each session for questions or issues that may be raised by these questionnaires. There are no physical risks with this research but some questions may bring up strong emotions.

Sign up sheets will be posted in the mental health services waiting area. When you sign up please put your name and DOC number in a space under the time period you would like to participate:

The sheets will then be posted in the dorms 2-4 days before the research sessions, confirming your choice of time and listing what room the research will be held in.

Maggie Zingman, MS (Doctoral Candidate)

Oklahoma State University 
APPENDIX D 


\section{Debriefing Sheet}

Thank you for participating in our research project. You may now realize that this research is investigating the way that childhood sexual abuse and family relationships affect adult mental health and symptom levels. The research project is called "Incarcerated Women: Impact of Childhood Sexual Abuse and Family Functioning on Dissociation and Psychological Distress."

We did not want to advertise this as a specific study on sexual abuse. If we had, any volunteer could/be identified as a sexual abuse survivor. We wanted to protect each participant's confidential information regarding such a history.

We realize that if you are a survivor of abuse or had a traumatic childhood, some of these questions may have brought up uncomfortable emotions. I have left an hour and a half after each session to briefly discuss these issues with individuals and can make referrals to Mental Health Services and Dr. Vinsant or one of his colleagues. As you know, you can also make requests to staff for counseling. For the post-session debriefing, please place your name on the list beside the door if you want to talk privately for a few moments or for a group debriefing after the private discussions. Please remember that there is no way I can identify anyone's answer sheet and thus, if I notice any answers that show. extreme distress or anxiety, I will be unable to identify who you are. If you have signed the back sheet, that you want some help or want to sign up for the post session brief group and individual counseling we will begin these after everyone is finished with the questionnaires.

Please keep your copy of your consent form. Results of this research should be analyzed in the next few months and the final research appear should be completed by May 2003. You can write or call the department in order to contact me to find out more about the results.

It is hoped that information we gain from this research will help develop more effective mental health interventions for female prisoners and for survivors in general.

Maggie Zingman, MS (Doctoral Candidate)

$\mathrm{C} / \mathrm{O}$

Counseling Psychology Department

Willard Hall $4^{\text {th }}$ Floor

Oklahoma State University

Stillwater, OK 74075

405 744-5000 
APPENDIX E 


\section{ATTENTION \\ ASSISTANCE REQUEST}

I feel I am experiencing overwhelming emotions after answering the four questionnaires. I feel I am in crisis and would like to talk to someone immediately. I understand that by signing this sheet, I ask that confidentiality be ended and my identity be known so I may receive help.

I understand that by signing this statement, I am stating that my reactions to the questions cannot be handled by the brief individual and group debriefing Ms. Zingman is providing after the session nor by a regular self-referral to EWCC Mental Health Services.

I understand that Ms. Zingman will either talk with me about my reactions or make a referral based on my answers about my emotional health. (Mark choices below)

If needed she will provide mental health Services with a short explanation of my crisis. I realize that depending on the severity of my response I may receive an immediate referral or one in a few days. Ms. Zingman will inform me of the possible time range but Dr. Vinsant will have the final recommendation.

I ask Ms. Zingman to talk with me (print name) state that I feel in crisis and

After the session during debriefings

Right now

Please just review $\mathrm{m}$ answers and make a referral in my name 
APPENDIX $\mathrm{F}$ 


\section{Participation Script}

Welcome. Thank you for participating in our research. As advertisements described, we are investigating how childhood family relationships affect adult coping styles. You have four tests in front of you. Each has an explanation on how to use the answer sheet. There are true or false questions which ask you to mark the appropriate box, some questions require a yes or no, a one or two word answer or a number value. These questions ask about your childhood and your primary caretakers from birth to 18 years old. You will also be asked about abuse history, emotions, and relationships with others. These questions should not cause any harm, but may bring up unpleasant memories or feelings. At the back of the test packet, there is a form to sign if, and only if, in answering questions, you begin to feel a need to talk with a staff therapist. If you sign this form, you release confidentiality so the therapist can assess your emotional issues. Only sign this if you want a referral. Beyond this release, all information gathered is kept confidential. At the front of the packet, you will notice the informed consent. Let's read it together. (read) Does everyone understand that your identity is protected by not having your name on answer sheets and that you have a right to leave this session if you become uncomfortable? (answer questions) Does everyone also understand that, if you don't want your consent form to be part of a group from which five samples will be drawn and sent to the Department of Corrections, you must check the box at the bottom of the informed consent? (answer questions). It takes about an hour to complete these questionnaires. Do not be concerned if someone finishes before you. Answer to the best of your ability. I can only answer questions about the correct way to mark the answer sheets but not how to interpret the question. When you are finished, please bring your packet forward. I will separate your answer sheets from your consent form, sign the consent form, and give you a sheet explaining the full purpose of the research. Again thank you for your help with this research. Begin. 


\section{Oklahoma State University Institutional Review Board}

Protocol Expires: $1 / 26 / 2004$

Date: Monday, January 27, 2003

IRB Application No ED0354

Proposal TTile: INCARCERATED WOMEN: IMPACT OF CHILDHOOD SEXUAL ABUSE AND FAMILY

DYNAMICS ON DISSOCIATION AND PSYCHOLOGICAL DISTRESS

Principal

Investigator(s):

Margaret Zingman

$3408 \mathrm{~S}, 121 \mathrm{E}$. Ave

Donald Boswell

Tulsa, OK 74146

406 Willard

Stillwater, OK 74078

Reviewed and

Processed as: Full Board

Approval Status Recommended by Reviewer(s): Approved.

Dear PI:

Your IRB application referenced above has been approved for one calendar year. Please make note of the expiration date indicated above. It is the judgment of the reviewers that the rights and welfare of individuals who may be asked to participate in this study will be respected, and that the research will be conducted in a manner consistent with the IRB requirements as outlined in section 45 CFR 46.

As Principal Investigator, it is your responsibility to do the following:

1. Conduct this study exactly as it has been approved. Any modifications to the research protocol must be submitted with the appropriate signatures for IRB approval.

2. Submit a request for continuation if the study extends beyond the approval period of one calendar year. This continuation must receive IRB review and approval before the research can continue.

3. Report any adverse events to the IRB Chair promptly. Adverse events are those which are unanticipated and impact the subjects during the course of this research; and

4. Notify the IRB office in writing when your research project is complete.

Please note that approved projects are subject to monitoring by the IRB. If you have questions about the IRB procedures or need any assistance from the Board, please contact Sharon Bacher, the Executive Secretary to the IRB, in 415 Whitehurst (phone: 405-744-5700, sbacher@okstate.edu).

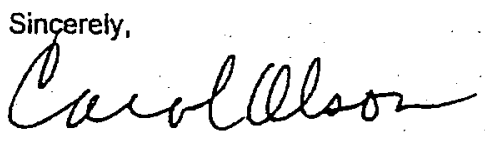

Carol Olson, Chair Institutional Review Board 


\section{VITA}

\section{Margaret Lee Zingman}

Candidate for Degree of

Doctor of Philosophy

Dissertation: INCARCERATED WOMEN: IMPACT OF CHILDHOOD SEXUAL ABUSE AND FAMILY FUNCTIONING ON DISSOCIATION AND PSYCHOLOGICAL DISTRESS

Major Field: Psychology

Biographical:

Education: Graduated from Barlow High School in Amenia, New York in May, 1973; received Bachelor of Arts Degree in Creative Writing from Eckerd College in St. Petersburg, Florida in January, 1979; received Master's of Science Degree in Community Counseling from Oklahoma State University in Stillwater, Oklahoma in December 1996; completed the requirements for the Doctor of Philosophy Degree with a major in Counseling Psychology from Oklahoma State University in Stillwater, Oklahoma December, 2003.

Experience: Worked as a therapist from 1986 until 1988 as Case Coordinator and Case Investigator/Crisis Specialist for abused children at Guardian ad Litem; worked as therapist specializing in adult trauma survivors from 1994 until 1998 at Women's Resource Center during practicum and periods between practicum; worked as therapist at the Psychological Service Center at Oklahoma State University for the first Ph. D. training practicum from 1996 through 1997; participated in four semesters of Family Clinic couple counseling training; have trained for community disaster intervention since 1990 and provided disaster intervention services for victims of the 1995 Murrow Building Bombing Disaster Site, the 1999 Bridgecreek Tornado and the 2003 Stroud Tornado; worked as therapist at Eddie Warrior Correctional Center and Eastern State Hospital from 2001 until 2002; returned to Women's Resource Center in 2002 until 2004.

Professional Memberships: American Psychological Association; International Society for the Study of Dissociation; Association of Traumatic Stress Specialists. 